\title{
Morphometric and microcirculatory aspects of muscle use and overuse
}

Citation for published version (APA):

Peeze Binkhorst, F. M. (1989). Morphometric and microcirculatory aspects of muscle use and overuse. [Doctoral Thesis, Maastricht University]. Rijksuniversiteit Limburg. https://doi.org/10.26481/dis.19891215fp

Document status and date:

Published: 01/01/1989

DOI:

10.26481/dis.19891215fp

Document Version:

Publisher's PDF, also known as Version of record

\section{Please check the document version of this publication:}

- A submitted manuscript is the version of the article upon submission and before peer-review. There can be important differences between the submitted version and the official published version of record.

People interested in the research are advised to contact the author for the final version of the publication, or visit the DOI to the publisher's website.

- The final author version and the galley proof are versions of the publication after peer review.

- The final published version features the final layout of the paper including the volume, issue and page numbers.

Link to publication

\footnotetext{
General rights rights.

- You may freely distribute the URL identifying the publication in the public portal. please follow below link for the End User Agreement:

www.umlib.nl/taverne-license

Take down policy

If you believe that this document breaches copyright please contact us at:

repository@maastrichtuniversity.nl

providing details and we will investigate your claim.
}

Copyright and moral rights for the publications made accessible in the public portal are retained by the authors and/or other copyright owners and it is a condition of accessing publications that users recognise and abide by the legal requirements associated with these

- Users may download and print one copy of any publication from the public portal for the purpose of private study or research.

- You may not further distribute the material or use it for any profit-making activity or commercial gain

If the publication is distributed under the terms of Article $25 \mathrm{fa}$ of the Dutch Copyright Act, indicated by the "Taverne" license above, 
MORPHOMETRIC AND MICROCIRCULATORY ASPECTS

OF MUSCLE USE AND OVERUSE. 


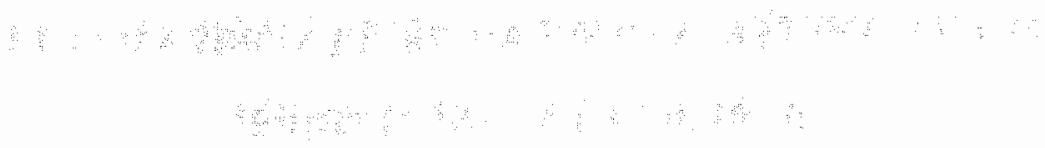




\section{MORPHOMETRIC AND MICROCIRCULATORY ASPECTS OF MUSCLE USE AND OVERUSE}

\section{PROEFSCHRIFT}

ter verkrijging van de graad van doctor aan de Rijksuniversiteit Limburg te Maastricht, op gezag van de Rector Magnificus, Prof. Dr. F.I.M. Bonke, volgens het besluit van het College van Dekanen,

in het openbaar te verdedigen op vrijdag, 15 december 1989 om 16.00 uur.

$$
\text { door }
$$

Frank Maurice Peeze Binkhorst

geboren te Weert in 1958. 
Promotor:

Co-promotores:

Beoordelingscomissie:
Prof. Dr. R.S. Reneman

Dr. H. Kuipers

Dr. G.J. Tangelder

Prof. Dr. F.T. Bosman, voorzitter

Prof. Dr. R.A. Binkhorst

Prof. Dr. J. Drukker

Prof. Dr. F. ten Hoor

Prof. Dr. W.L. Mosterd

\section{CIP-DATA KONINKLIJKE BIBLIOTHEEK, DEN HAAG}

Peeze Binkhorst, Frank Maurice

Morphometric and microcirculatory aspects of muscle use and overuse / Frank Maurice Peeze Binkhorst ; [ill. by the author]. Maastricht : Datawyse. - Ill.

Thesis Maasticht. - With ref. - With summary in Dutch. ISBN 90-5291-015-4 SISO 599.7 UDC 612.73/.74 (043.3)

Subject headings: muscle use; morphometric aspects / muscle use ; microcirculatory aspects.

De uitgave van dit proefschrift en de promotie werden mede mogelijk gemaakt door financiele steun van CENTOCOR EUROPE B.V. 
"When you picked up this book, did it occur to you that it was those obediant servants, your muscles, that actually made your action possible?" (Squire, 1981).

Voor mijn ouders, voor Eline, en voor wat komen gaat. 
2. MUSCLE MORPHOLOGY AND PHYSIOLOGY 5

2.1 muscle fiber types 5

2.2 muscle blood supply 8

2.3 muscle fiber adaptation 10

2.4 muscle fiber overload and necrosis 10

3. EXERCISE-INDUCED FOCAL SKELETAL MUSCLE FIBER DEGENERATION AND CAPILLARY MORPHOLOGY 15

3.1 Introduction 15

3.2 Materials and methods 16

$\begin{array}{lll}3.3 & \text { Results } & 19\end{array}$

3.4 Discussion 27

$\begin{array}{lll}3.5 & \text { Summary } & 30\end{array}$

4. CAPILLARY (ULTRA)STRUCTURE IN RELATION TO POSITION AND FIBER TYPE IN RAT SOLEUS MUSCLE 31

4.1 Introduction 31

4.2 Materials and methods $\quad 32$

4.3 Results 37

4.4 Discussion 41

4.5 Summary 43

5. EXERCISE INDUCED SWELLING OF RAT SOLEUS MUSCLE. ITS RELATION WITH INTRAMUSCULAR PRESSURE

5.1 Introduction 45

5.2 Materials and methods $\quad 46$

5.3 Results 51

5.4 Discussion 54

5.5 Summary 57 
6. FIBER TYPE RELATED THICK FILAMENT SPACING IN PLASTIC EMBEDDED MUSCLE 59

6.1 Introduction 59

6.2 Materials and methods 60

6.3 Results 64

$\begin{array}{lll}6.4 & \text { Discussion } & 70\end{array}$

6.5 Summary 74

6.6 Appendix 75

7. FLUORESCENT LABELING OF CAPILLARY ENDOTHELIUM INVTVO

$\begin{array}{lll}7.1 & \text { Introduction } & 77\end{array}$

$\begin{array}{lll}7.2 & \text { Materials and methods } & 78\end{array}$

$\begin{array}{lll}7.3 & \text { Results } & 82\end{array}$

$\begin{array}{lll}7.4 & \text { Discussion } & 86\end{array}$

7.5 Summary 89

8. MEASUREMENT OF SKELETAL MUSCLE CAPILLARY DIAMETERS IN VIVO 91

8.1 Introduction 91

8.2 Materials and methods 92

8.3 Results 94

$\begin{array}{lll}8.4 & \text { Discussion } & 96\end{array}$

$\begin{array}{lll}8.5 & \text { Summary } & 98\end{array}$

9. GENERAL DISCUSSION 99

10. REFERENCES 103

11. SUMMARY 111

12. NEDERLANDSE SAMENVATTING 115

CURRICULUM VITAE $\quad 125$

PUBLIKATIES $\quad 126$

NAWOORD 128 
× $\quad \therefore$ 


\section{GENERAL INTRODUCTION}

Most skeletal muscles are used to move the body. They can function in different ways. For example, muscle contraction can be fast, like in sprinting, powerful, like in weight lifting, or muscles can be active for long periods of time, like in marathon running. Although muscles can in general adapt easily to changes in work load, unaccustomed exercise may overload a muscle and lead to damage of the muscle fibers (Geller, 1973; Lindholm et al., 1974; Bartsch et al., 1977; Fridén et al., 1983; Armstrong et al., 1983; Salminen and Vihko, 1984A; Warhol et al., 1985; for review see Hoppeler, 1986). In animals, the damaged muscle fibers often become invaded by cells that appear to remove the debris and contribute to the regeneration of the degenerated fibers.

Kuipers and colleagues (1983) have investigated exercise induced muscle fiber degeneration in more detail in the rat. They found that $1 \mathrm{~h}$ uphill running at a moderate speed $(1.8 \mathrm{~km} / \mathrm{h})$ caused fiber degeneration and invasion by inflammatory cells $24-48 \mathrm{~h}$ after running in some muscles, whereas other muscles remained unaffected. The degenerated fibers were situated between morphologically unaffected fibers, and only short segments of the affected fibers $(150-1250 \mu \mathrm{m}$; total fiber length is about $2 \mathrm{~cm}$ ) were degenerated and invaded. Of all the seven hind limb muscles investigated, the soleus muscle was most severely affected by the running procedure with $2-5 \%$ of its fibers demonstrating signs of degeneration. Within the soleus muscle, only slow twitch (type I) muscle fibers were affected wereas fast twitch (type II) fibers appeared to be normal.

Although morphological changes in muscle fibers after unaccustomed exercise have been discribed extensively in humans as well as animals (see chapter 2), little is known about the actual cause and mechanism involved in the muscle fiber degeneration. It is striking that the length of the degenerated segment of the affected fibers $(150-1250 \mu \mathrm{m})$ is similar to the length of individual muscle capillaries, i.e., 200-1000 $\mu \mathrm{m}$ (Erikson and Myrhage, 1972; Myrhage and Hudlicka, 1976; Dawson et al., 1987; Wiederhielm and Slaaf, 1987). This could indicate an obstruction of certain capillaries, e.g. due to sticking of blood cells or swelling of endothelial cells, 
resulting in ischemia and damage of the fiber segment they supply. In addition to such a local ischemia, obstructions at the arterial or veinous side of the vascular bed might cause ischemia in larger groups of muscle fibers, which could result in damage of the most vulnerable fibers within the muscle.

The main aim of the present study present study was to investigate whether or not, and, if so, to which extent, ischemia plays a role in exercise induced muscle fiber degeneration (chapters 3,4 and 5). The experiments were performed on the soleus muscle of rats folowing the runining protocol discribed by Kuipers et al. (1983). This muscle was used because it is the one most affected by the running

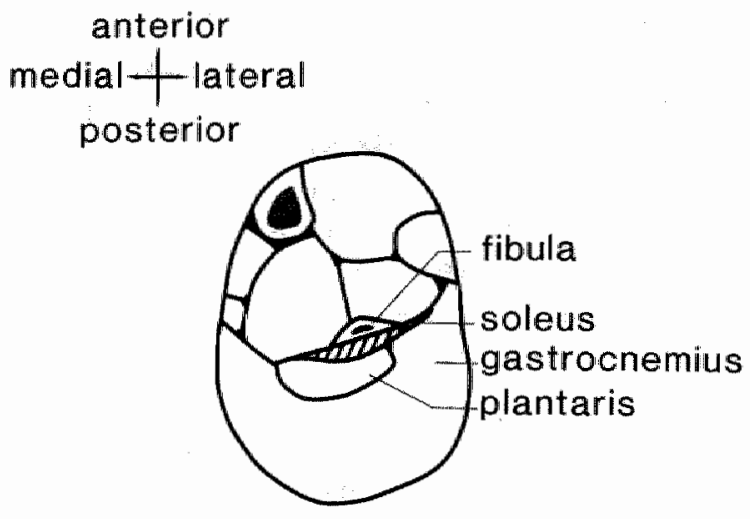

Fig. 1.1. Cross section of the rat lower hind limb. The soleus muscle is located in the center of the limb, being bordered by the fibula and the gastrocnemius and plantaris muscles.

procedure. The soleus muscle of the rat is located in the lower hind limb posterior to the fibula and anterior to the gastrocnemius and the plantaris muscle (Laughlin and Armstrong, 1983; see Figure 1.1). It controls, in co-operation with other muscles, the position of the foot and is in that way continuously involved in the maintainance of bearing (Henneman and Olson, 1965). More specifically we addressed the following aspects:

1.- Is exercise induced muscle fiber degeneration preceeded by capillary morphological changes indicative of ischemia? Such changes might involve capillary obstruction, e.g. plugging by leukocytes, a decrease in capillary diameter or changes in endothelial structure influencing transcapillary exchange. Morphological analysis of capillary cross-sections at the light and electron microscopic level was used to detect possible capillary obstructions and to search for general and local capil- 
lary abnormalities, if any; in exercised rats and in control animals that did not run (chapter 3).

2.- Slow twitch (type I) fibers of the rat soleus muscle are more vulnerable to uphill running than fast twitch (type II) fibers (Kuipers et al., 1983). If the fiber degeneration is caused by ischemia, it is conceivable that the more vulnerable fiber types are supplied with relatively fewer capillaries than the less vulnerable fiber types, or that their capillaries are small in diameter or less well adapted to efficient exchange of substances. If both fiber types would be supplied by a similar amount of capillaries of similar morphology, than a difference in the susceptibility between slow and fast twitch fibers to uphill running would be expected. For this study a method was developed to classify fibers in cross-sections of plastic embedded muscles. Capillary morphometry was investigated in relation to fiber type, using light and electron microscopy (chapter 4).

3.- Is exercise induced muscle fiber degeneration preceeded by swelling of the muscle and, if so, does this swelling lead to an increase in tissue pressure sufficient to impair blood flow? Fiber swelling was determined by weighing muscles and by measuring muscle fiber cross-sectional areas after running as well as in non-exercised controls. Tissue pressure was determined with the use of a relatively atraumatic servocontrolled micropipette technique (chapter 5).

During the experiments in which muscle fibers were classified in cross-sections of plastic embedded muscle (chapter 4), it was observed that slow twitch (type I) fibers appeared lighter in sections stained with toluidine blue as compared to fast twitch (type II) fibers. Therefore, we investigated in chapter 6 to what extent fibers could be classified in plastic sections on the basis of light absorption. Because it is conceivable that the light absorption is related to spacing of the contractile filaments, we also studied filament spacing in the different fiber types. Filament spacing might not only be related to the strength and speed of contraction of a fiber, it might also be used for classification of muscle fibers.

Because in vivo observation would be a more direct approach to study the various changes in perfusion of the muscle, in the two final chapters an attempt was made to investigate capillary morphology in vivo. Since it is extremely difficult to visualize capillaries in skeletal muscles by means of regular bright field microscopy, especially in thick muscles, we tried to find a fluorescent dye that labels the endothelium in order to improve the visualization of capillaries in the soleus muscle in vivo (chapter 7). In the final experimental chapter (8) we determined capillary inner diameters in the rat soleus muscle and compared them with those obtained from plastic cross-sections.

A variety of techniques were required to solve these questions. Therefore, the methods used are not presented in a separate chapter, but are described in detail in the appropriate chapters. 
The experimental chapters are preceeded by an introduction in skeletal muscle anatomy, physiology and exercise induced pathology (chapter 2). The thesis ends with a general discussion in which the results described in the experimental chapters (3-8) are put into perspective (chapter 9). 
Skeletal muscles constitute about $40 \%$ of total body mass and form the largest organ system of the vertebrate body (Hoppeler, 1987). They allow the body to move and thus provide the organism with unique possibilities to interact with its environment. The evolution of skeletal muscles allows the exploitation of new food sources and opens new ways to the species to secure and multiply its genes. In addition, muscles created unique possibilities for social interaction between individuals. In this way, these muscles have made possible new evolutionary developments throughout the ascent of animals and man.

Skeletal muscles are adapted to their specific function, or rather, are able to exert a specific function because they have certain anatomical and physiological characteristics. For example, fast muscles are used for sprinting, but they fatigue quickly. In contrast, muscles like those involved in bearing are slow but capable of maintaining their activity for a long period of time.

The contractile units of the muscle are the sarcomeres of the muscle fibers. Muscle fibers, which can be regarded as long strands of fused cells (syncytia), are organized in parallel bundles. Muscles, muscle bundles and muscle fibers are enveloped by connective tissue sheats, called epi-, peri- and endo-mysium, respectively. The connective tissue contains the nerves that control the muscles, as well as the blood and lymph vessels.

\subsection{MUSCLE FIBER TYPES}

Muscle fibers can basically be divided into slow and fast twitch fibers. Slow twitch fibers can be active for long periods of time without getting fatigued. However, the strength development by these fibers is limited and fast twitch fibers have to be recruited when more power and higher velocities of contraction are required. Fast twitch fibers can be divided into fibers that can work for longer periods of time, and fibers that are quickly fatigued (Garnett et al., 1978; Thornell et al., 1987). 


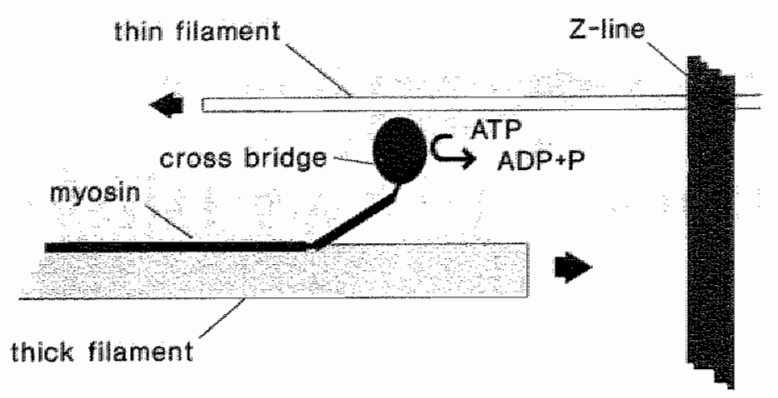

Fig. 2.1 Schematic drawing of a myosine molecule of a thick filament and an adjacent thin filament. The ATPase activity is situated on the head of the myosin molecule.

Slow and fast twitch muscle fibers can be discriminated histochemically in muscle cryosections, because their myosin ATPase activity is inhibited at certain pH levels in a different manner. Myosin ATPase activity is located on the head of the myosin molecule (see Fïgure 2.1), and is used to release from ATP (adenosine triphosphate) molecules the energy necessary for contraction. The ATPase activity of slow twitch fibers (type $\mathrm{l}$ ) is resistent to preincubation at $\mathrm{pH}=4.3$, but ATPase activity of fast twitch fibers (type II) is inhibited after preincubation at this $\mathrm{pH}$. Fast twitch fibers can be fatigue resistant or can be quickly exhausted. These fibers can be distinguished histochemically and are called IIA and IIB fibers, respectively. Only the myosin ATPase activity of type IIA fibers is inhibited at $\mathrm{pH}=4.6$ (Brook and Kaiser, 1970; Dubowitz, 1985). Type IIC fibers can also be distinguished and are considered. to be undifferentiated or intermediate between type IIA and type I fibers (Howald et al. 1985; Staron et al., 1984; Staron and Pette, 1986; Maier et al., 1988). Using subtle differences in myosin ATPase sensitivity to $\mathrm{pH}$, several fiber types intermediate between type I, IIA, IIB and IIC can be distinguished as well (Staron et al., 1983; Staron and Pette, 1986; Maier at al, 1988). However, the physiological relevance of these subdivisions is debatable and, consequently, they are usually deleted.

The high endurance capacity of slow twitch type I, as compared to fast twitch type IIB fibers, can at least in part be explained by their different blood supply and metabolic pathways. The metabolism of type I fibers is highly dependent on oxygen (aerobic or oxidative metabolism). Because type I fibers are well supplied with capillaries, in contrast to type IIB fibers (see Figure 2.2) (Andersen, 1975; Andersen and Kroese, 1978; Gray and Renkin, 1978; Hudlická et al., 1982; Smith et al., 1989), oxygen and waste products are rapidly exchanged and the fiber can work for long periods of time without getting fatigued. Type IIB fibers function mainly without oxygen (anaerobic or glycolytic metabolism) and consequently produce relatively large amounts of lactate when active. Accumulation of lactate and the limited exchange capacity as a result of a low degree of capillarization (i.e. low number of ca- 

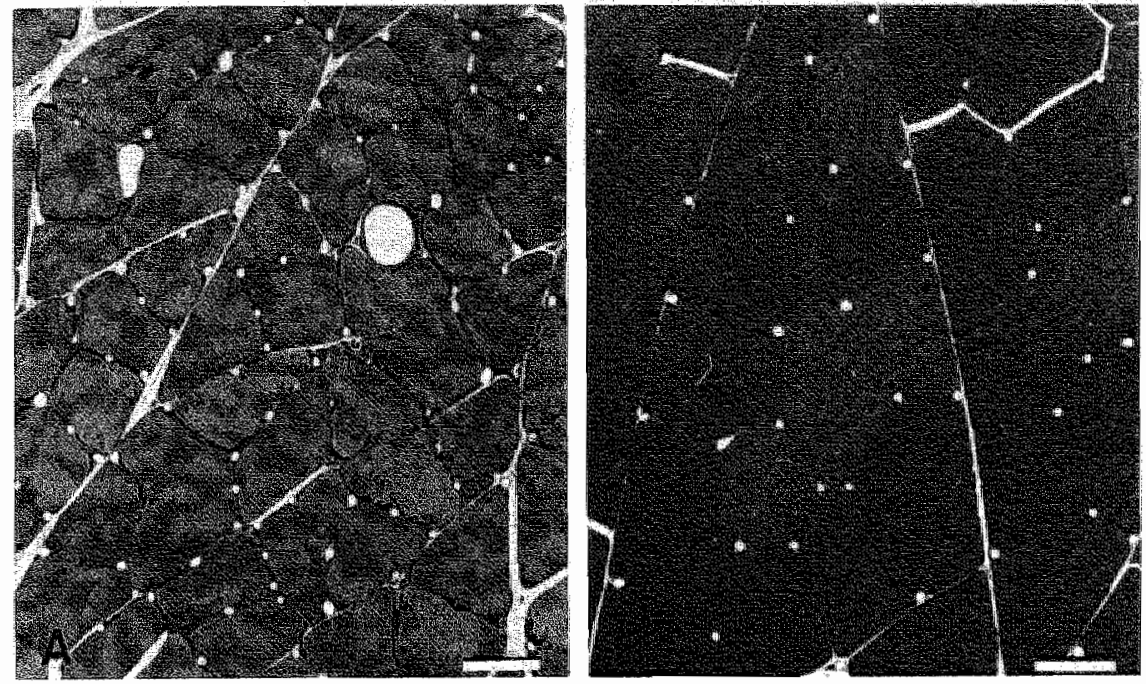

Fig. 2.2. Cross-sections of plastic embedded muscles of rat, fixed by perfusion. (A) Soleus muscle. About $80 \%$ of the fibers are slow (type I $\$$. None of the fibers use a predominantly glycolytic metabolic pathway. The soleus muscle is supplied by many capillaries per cross-sectional area. (B) White part of the gastrocnemius muscle. Virtually all fibers are fast (type II). About $90 \%$ of the fast fibers use a predominantly glycolytic metabolic pathway (generally type IIB) (Laughin and Armstrong; 1983). The gastrocnemius muscle is supplied by few capillaries per cross-sectional area. Bars, $25 \mu \mathrm{m}$.

pillaries per unit cross-sectional fiber area) can explain the low fatique resistance of type IIB fibers. The degree of capillarization of type MA fibers is variable, but appears to be intermediate between that of type I and type IIB fibers. Type IIA fibers can use oxidative as well as glycolytic metabolic pathways. Because different metabolic pathways are used by type I, IIA and IIB fibers, they are often referred to as Slow Oxidative (SO), Fast Oxidative Glycolytic (FOG) and Fast Glycolytic (FG), respectively. The metabolic properties of muscle fiber types are reflected in differences in the activity of oxidative enzymes present in their mitochondria, e.g. succinate dehydrogenase, or in the activity of enzymes associated with anaerobic metabolic pathways, e.g. lactate dehydrogenase. The activity of enzymes associated with an aerobic or a glycolytic pathway can be demonstrated histochemically and can be used for metabolic classification of muscle fibers (Peter et al., 1972; Nemeth and Pette, 1981; Staron et al., 1984; Hoppeler, 1987). The main differences between the fiber types mentioned are summarized in Table 2.1. 
Table 2.1. Properties of fiber types.

\begin{tabular}{|c|c|c|c|}
\hline FIBER TYPE & I & IIA & IIB \\
\hline $\begin{array}{l}\text { Contractile properties: } \\
\text { velocity } \\
\text { strength } \\
\text { fatigue resistance }\end{array}$ & $\begin{array}{l}\text { slow } \\
\text { weak } \\
\text { high }\end{array}$ & $\begin{array}{l}\text { fast } \\
\text { strong } \\
\text { high }\end{array}$ & $\begin{array}{l}\text { fast } \\
\text { strong } \\
\text { low }\end{array}$ \\
\hline Metabolic properties: & oxidative & $\begin{array}{l}\text { oxidative/ } \\
\text { glycolytic }\end{array}$ & glycolytic \\
\hline $\begin{array}{l}\text { ATPase activity after } \\
\text { pre-incubation at : } \\
\text { pH } 4.3 \\
\text { pH } 4.6\end{array}$ & + & - & + \\
\hline Similar to fiber type: & $\mathrm{sO}$ & $\mathrm{FOG}$ & $\mathrm{FG}$ \\
\hline
\end{tabular}

$S O=$ slow oxidative, $F O G=$ fast oxidative glycolytic, $F G=$ fast glycolytic.

$+=$ present,$-=$ suppressed.

In addition to their myosin ATPase activity and main metabolic pathways, slow and fast twitch muscle fibers can differ biochemically and morphologically. Biochemical differences in their contractile proteins, like myosin, can be demonstrated using electrophoresis of slow and fast twitch muscles (Lowey and Risby, 1971) or of isolated slow and fast twitch muscle fibers (Staron and Pette, 1986; Eddinger and Moss, 1987). Fiber type related biochemical differences can also be demonstrated using antibodies that can discriminate between contractile filaments of slow and fast twitch muscle fibers (Pierobon-Bormioli et al., 1981; Moore et al., 1984; Sawchak et al., 1985). Structural differences, like those present in the M-line and Z-band, can be demonstrated using electron microscopy (Sjöström et al., 1982; Thornell et al., 1987 ; c.f. Squire, 1981).

\subsection{MUSCLE BLOOD SUPPLY}

In vertebrates, blood is supplied to and distributed within the muscles via blood vessels. Blood flow in a muscle is regulated mainly at the level of the arterioles, which are the most important resistant vessels (Tangelder et al., 1984; Davis, 1988). Exchange of substances between blood and the muscle fibers takes place at the level of the capillaries and the smallest arterioles and venules. These substances include 
oxygen and energy rich molecules used for the production of ATP, hormones and ions, and waste products of the muscle fibers like carbon dioxide and lactic acid.

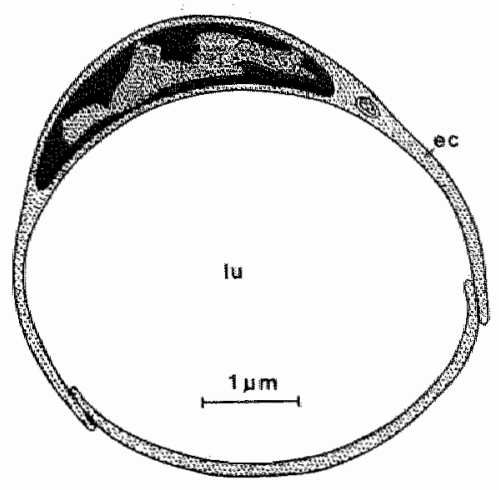

Fig. 2.3. Schematic drawing of a capillary cross-section. Two flat endothelial cells can be discriminated. The endothelial cells are attached to each other at their edges that overlap slightly. $E C=$ endothelial cell, $L U=$ lumen.

In general, the capillaries run parallel to the muscle fibers when the muscle is stretched, but meander when the muscle is contracted (Potter and Groom, 1983). Their length is about 200-1000 $\mathrm{m}$ (Erikson and Myrhage, 1972; Myrhage and Hudlicka, 1976; Dawson et al., 1987; Wiederhielm and Slaaf, 1987). Capillaries are composed of flat endothelial cells, the major constituents of their wall (Figure 2.3). In capillary cross-sections generally one or a few endothelial cells can be distinguished. Capillaries are only a few microns in diameter (Myrhage and Hudlicka, 1976; Dawson $e$ t al., 1987). Red and white blood cells are generally deformed when they are squeezed through the narrow tubes, and especially leukocytes can easily block capillaries, especially when the driving pressure is limited (Erikson and Myrhage, 1972; Myrhage and Hudlicka, 1976; Bagge and Braide, 1982). Following damage of muscle fibers, leukacytes can leave the capillary lumen through the interendothelial clefts, mainly in the venules, and enter the affected region (Kuipers et al., 1983; Salminen and Vihko, 1984A). Transport of substances across the capillary wall takes place through diffusion via the endothelial membranes or the inter endothelial clefts and possibly via endothelial vesicles (Simionescu et al., 1975; Casley-Smith, 1981; Yokota, 1983; Orgnero de Gaisán et al., 1985). 


\subsection{MUSCLE FIBER ADAPTATION}

Muscles can vary greatly from individual to individual as far as their morphological and functional properties are concerned. Often these differences are associated with variations in functional demands, indicating that muscles can be trained. When the work load of a muscle is changed, muscle fibers can adapt within days or weeks (Luginbuhl, 1984; Howald et al., 1985; Hoppeler, 1986). If the change in work load is small, only the endurance capacity may change and fibers can shift towards a more aerobic (oxidative) or a more anaerobic (glycolytic) metabolism without any alteration in fiber type, as assessed by myosin ATPase activity (Luginbuhl et al., 1984; Hoppeler, 1987). If the change in work load is more pronounced, also changes in contractile properties might occur. Muscle fibers can then change from one fiber type to another by changing their myosin light and/or heavy chains. For example, type II muscle fibers usually change into type I fibers (IIB --> IIA -- > I via intermediate fiber types), if they are activated for longer periods of time, as in endurance training (Luginbuhl et al., 1984; Howald et al., 1985). A similar change is observed with chronic electrical stimulation (Rubinstein et al., 1978; Maier et al., 1988). On the other hand, type I fibers can change into type II fibers as a result of high-intensity intermittent training or inactivity (Hoppeler, 1987). However, if the intensity or the duration of a work load is too high and/or too long for a muscle, damage may occur.

The changes in contractile and metabolic properties due to training and detraining are often reflected in fiber morphology. For example, endurance training results in an increase in the number of mitochondria, especially in the number of pericapillary, subsarcolemmal mitochondria (Howald et al., 1985; Hoppeler, 1986 and 1987), and in the amount of lipid (Staron et al., 1984; Howald et al., 1985; Hoppeler, 1986) and glycogen (Hoppeler, 1986) stored in the muscle fibers. Chronical electrical stimmulation can change the Z-band width from that characteristic of a fast twitch fiber type (narrow Z-band) to that characteristic of slow twitch fibers (wide $\mathrm{Z}$-band) (Salmons et al., 1978). The main effect of strength training is muscle fiber hypertrophy and/or fiber hyperplasia (Gonyea et al., 1976; Hoppeler, 1986). In addition to changes in the muscle fibers, the number of capillaries per muscle and muscle fiber cross-sectional area increases by endurance training (Andersen, 1975; Inger and Brodal, 1978; Tornling, 1982). Chronic electrical stimulation can also increase capillary density (Cotter et al., 1973; Hudlická et al., 1982; Cabric et al., 1987)

\subsection{MUSCLE FIBER OVERLOAD AND NECROSIS}

Most people are familiar with the experience of muscle soreness which appears $24-48 \mathrm{~h}$ after heavy or prolonged unaccustomed exercise. Although the pain per- 
ception and its delayed appearance is still incompletely understood, they indicate that changes occur in overused muscle. Unaceustomed exercise can lead to muscle fiber swelling (Gonzales-Serratos et al., 1978; Fridén et al., 1988) and compartmental syndromes (Reneman, 1968 and 1975; Matsen, 1975; Fridén et al., 1988). Muscle fiber damage has been observed in humans (Geller, 1973; Friden et al., 1983; Warhol et al., 1985; for review see Hoppeler, 1986) as well as in animals (Lindholm et al., 1974; Bartsch et al., 1977; Kuipers et al., 1983; Armstrong et al., 1983; Salminen and Vihko, 1984). Different kinds of exercise may cause damage to different muscles. Within a muscle the slow twitch or fast twitch fibers, or even both fiber types, may be affected (Kuipers et al., 1983; Fridén et al., 1983 and 1988; Fridén, 1984). The damage is usually local, i.e., in between apparently unaffected fibers, and focal, i.e. only part of the fiber is affected. The damage may consist of disruption of the $Z$-line of the sarcomere (Z-line streaming), disorganization of myofibrils, instable and/or disrupted mitochondrial membranes, and endothelial cell damage and swelling (Fridén et al., 1981, 1983 and 1988; Kuipers et al., 1983; Armstrong et al., 1983; Fridén, 1984; Warhol et al., 1985; Hoppeler, 1986). In animals it has been established that muscle fiber damage is associated with an increase in lysosomal proteolytic enzyme activity in the affected muscle (Salminen and Vihko, 1983; Salminen et al., 1984B; c.f. Fridén, 1984) and with the invasion of inflammatory cells. Invasion of the muscle fiber is most pronounced $24-48 \mathrm{~h}$ after the onset of the damage (Kuipers et al., 1983; Salminen and Vihko, 1984A; Stauber et al., 1988). The invasive cells include macrophages, lymphoid cells and myoblasts (Kuipers et al., 1983; Stauber et al., 1988). Muscle fiber damage is reflected by an increase in myoglobin and muscle enzymes in the blood (Siegel et al., 1981; Schwane et al., 1983; Byrnes et al., 1985). Excessive myoglobinuria may cause renal failure (Hamilton et al., 1972). Sometimes extensive exercise induced fiber damage can even lead to death, as has been reported in man (Bartsch et al., 1977; c.f. Hamilton et al., 1972) and in animals, e.g., race horses (Smith and Jones, 1966) or chased wild animals (Bartsch et al., 1977). However, in general muscle fiber degeneration is followed by complete fiber repair within a few weeks (Kuipers et al., 1983; Salminen and Vihko, 1984A).

At present the primary cause of the focal damage and the mechanisms involved in the sequence of events leading to muscle fiber degeneration and regeneration have not been established. In particular, the involvement of microcirculatory events, if any, has to be established. Mechanical forces may contribute to changes in muscle fiber structure, as is indicated by the rupture of the Z-band of sarcomeres following unaccustomed exercise (Fridén et al., 1981 and 1983; Fridén, 1984). If other cellular structures are ruptured, e.g. membranes of the muscle fibers, a series of processes can be initiated. Muscle enzymes may be released from the fibers into the interstitium and enter the circulation. This might explain the transiently elevated blood plasma levels of muscle fiber proteins found after exercise (Siegel et al., 1981; Schwane et al., 1983; Byrnes et al., 1985). Leukotactic factors might be released from the damaged fibers and attract leukocytes. Intracellular substances become available as a trigger for the immune system which may further damage the fiber. Sub- 
sequently, damaged cell structures might be removed by leukocytes and muscle regeneration might be completed by myoblasts (Sanderson et al., 1975; Stauber et al, 1988 ).

Several author have considered ischemia as a possible cause of exercise induced muscle fiber degeneration (Reneman, 1968 and 1975; Matsen, 1975; Salminen and Vihko, 1983 and 1984A). Indeed ischemia causes histological changes similar to those observed in unaccustomed extensive exercise. It can cause membrane damage (Ishiharajuma et al, 1986), swelling, and degeneration and invasion of muscle fibers (Sanderson et $a l$, 1975; Mäkitie and Teräväinen, 1977; DiBona and Powell, 1980). Ischemia may lead to ATP depletion and/or intoxication of muscle fibers and endothelium due to accumulation of breakdown products. Intoxication might inhibit the function of Ion pumps or induce membrane damage which might both lead to accumulation of cations within muscle fibers and endothelial cells, resulting in water uptake and swelling. Because only a few hours of ischemia are sufficient to cause muscle fiber degeneration (Sanderson et al., 1975; Mäkitie and Teräväinen, 1977), it seems likely that if ischemia is involved, it will be most pronounced several hours before fiber degeneration can be observed.

Ischemia can be general, i.e. occur in the whole muscle, or local. Both types of ischemia might theoretically induce the focal muscle fiber necrosis observed after exercise.

Generalized muscle ischemia can result in damage of the most vulnerable fibers or fiber segments. Exercise may cause swelling of muscles. If this swelling occurs in a low compliant space it may cause an increase in tissue pressure which could inhibit blood flow (Ashton, 1975; Reneman et al., 1980) and result in ischemia and compartmental syndromes (Reneman, 1968 and 1975; Matsen, 1975; Fridén et al., 1988). Muscle ischemia by itself can result in swelling of myofibers (Sanderson et al., 1975; DiBona and Powell, 1980) and might in this way aggravate the ischemia.

Local ischemia could result from a locally hampered capillary blood supply. One of the arguments in favor of this hypothesis is that the length of the focal degeneration is about 150-1250 $\mu \mathrm{m}$ (Kuipers et al., 1983), similar to the length of individual capillaries, 1.e., 200-1000 $\mathrm{m}$ (Erikson and Myrhage, 1972; Myrhage and Hudlicka, 1976;, Dawson et al., 1987; Wiederhielm and Slaaf, 1987). It is possible that the needs of some of the muscle fibers are limited at rest, but that they are too high to be redeemed by the microcirculation during or after unaccustomed exercise. This could happen if, for example, the number of capillaries surrounding the fiber is relatively low or if their humen is relatively small. In addition, local obstructions of the capillary lumen may reduce blood flow. Local damage and swelling of endothelial cells has been demonstrated in humans after marathon running (Warhol et al., 1985). Nuclei of endothelial cells can obstruct the lumen of microvessels in frog mesentery and skeletal muscle after electrical, chemical and mechanical stimulation (Weigelt et al, 1981; Tyml and Weigelt, 1982; c.f. Addicks et al., 1979). It is possible that similar processes occur as a result of exercise. In addition, blood cells, and in particular white blood cells, might obstruct capillary lumina, especially at low blood pressure (Bagge and Braide, 1982). Ischemia by itself can also induce endothelial 
swelling and disruption (Mäkitie, 1977; Gidlöf et al, 1987B; c.f. Sanderson, 1975) and might also in this way induce a vicious circle aggravating the ischemia. Changes of the capillary wall might also inhibit the exchange of material between blood and muscle fibers. For example, swelling of the endothelium and/or basal membrane, increased resistance of the inter-endothelial clefts, inhibition of the vesicular system and a reduction of ATP necessary for energy demanding transport processes might all impair transcapillary exchange. This could cause functional ischemia resulting in muscle fiber necrosis.

In summary, overuse of skeletal muscle may lead to local and focal muscle fiber damage and invasion by inflammatory cells. Little is known about the actual cause of the exercise induced inflammatory response. The histological changes that occur after muscle overuse are similar to those observed after muscle ischemia. In addition, the length of the affected muscle fiber area is similar to the length of muscle capillaries, indicating that ischemia at the capillary level might be involved in the degenerative process. This leads to the main aim of the study presented in this thesis, i.e. to investigate whether ischemia can be held responsible for the exercise induced focal muscle fiber degeneration. 


\section{EXERCISE-INDUCED FOCAL SKELETAL MUSCLE FIBER DEGENERATION AND CAPILLARY MORPHOLOGY}

\subsection{INTRODUCTION}

Focal degenerative and inflammatory changes, after heavy exercise of short duration or prolonged exercise of moderate intensity, have been reported in skeletal muscle of man (Geller, 1973; Warhol et al., 1985; for review, see Jerusalem et al., 1974A) and several animal species (Lindholm et al., 1974; Armstrong et al., 1983; Kuipers et al., 1983). The inflammatory response, as characterised by invasion of monomorphonuclear leukocytes, reaches its maximum 1-3 days after exercise, and coincides with maximal muscle soreness in humans (Asmussen, 1965; Schwane et al., 1983; Kuipers et al., 1985). The degenerative changes are usually followed by complete regeneration (Fridén et al., 1981, Warhol et al., 1985).

The mechanism of exercise-induced muscle fiber degeneration with its focal appearance is still incompletely understood. Focal muscle fiber degeneration similar to that found after exercise, has also been reported after a prolonged period of ischemia (Mäkitie and Taräväinen, 1977) and in compartmental syndromes due to exercise (Reneman, 1968 and 1975). Morphological changes in skeletal muscle capillaries, i.e., loss of cytoplasmic matrix and intercellular constituents, have been observed in humans after a prolonged period of running (Warhol et al., 1985). It is conceivable that changes at the microcirculatory level may lead to blood flow obstruction and/or impaired exchange of nutrients and waste products. If changes in the microcirculation are responsible for the degenerative process, postexercise focal muscle fiber degeneration may be associated with changes in capillary morphology interfering with blood flow and/or exchange of substances between plasma and myocytes.

It was the aim of the present study to investigate the effect of nonexhaustive uphill running on the capillary morphology in rat soleus muscle. Morphological and 
morphometric parameters, which may reflect changes in capillary morphology associated with capillary blood flow and exchange, were determined on a focal, i.e., close to the degenerated muscle fibers, and a general basis. Rat soleus muscle was chosen as a model, because in this musele uphill endurance running resulted in moderate focal changes (Kuipers et al., 1983), allowing the investigation of capillaries adjacent to degenerated fibers or surrounded by unaffected fibers.

\subsection{MATERIALS AND METHODS}

\section{Animals and exercise}

The study was performed on male Wistar rats. The animals were kept in a temperature-controlled environment $\left(23-26^{\circ} \mathrm{C}\right)$ and fed with rat chow (type 1210 , Hope Farms) and water ad libitum. At the age of 12 wk, the rats, weighing $290-330 \mathrm{~g}$, were randomly assigned to a nonexercised control group ( $\mathrm{C}$ group) or an exercise group. The rats of the exercise group were placed on a rodent treadmill (Quinton) with a positive slope of $10^{\circ}$, and they were stimulated to run by a mild electrical current (duration $0.1 \mathrm{~s}$ ) that was applied to the resting grid each $5 \mathrm{~s}$. Mean effective running during an initial $15 \mathrm{~min}$ period of adaptation $(875 \mathrm{~m} / \mathrm{h})$ was about $100 \%$ and during the following exercise period $(60 \mathrm{~min}$ at $1,750 \mathrm{~m} / \mathrm{h})$ about $50 \%$. Rectal temperature of the animals was measured (Thermovit 4400, Amos) before and immediately after running to estimate the degree of exhaustion (Gollnick and Ianuzzo, 1968). Room temperature (RT) during running was $23-26^{\circ} \mathrm{C}$.

\section{Perfusion and fixation procedures}

Before (Cgroup) and 0.05-1.11 hour (0-h group), 4.59-6.50 hours (6-h group) or 23.22-24.31 hours (24-h group) after running, animals were anesthetized with pentobarbital sodium (Narcovet;60\% solution;1 $\mathrm{ml} / \mathrm{kg}$ body wt ip). The abdomen was opened and heparin $(1,000 \mathrm{IU} / \mathrm{kg}$ body wt) was injected into the liver to prevent blood coagulation. Additional pentobarbital sodium $(60 \%, 0.3 \mathrm{ml} / \mathrm{kg}$ body wt) was dripped into the abdominal cavity for additional anesthesia. Five minutes after the injection of heparin, the abdominal aorta was ligated just distal to the renal arteries.

For perfusion of the left hindlimb, a silicone cannula (602-155; Silastic; Dow Corning, USA) was inserted into the abdominal aorta and fixed with the tip positioned a few millimeters from the iliac bifurcation. The left leg was fixed in a standard position (ankle $60^{\circ}$, knee $90^{\circ}$, hip $90^{\circ}$ ). The right iliac artery was clamped to prevent flushing and fixation of the right leg. The cannula was subsequently connected to the perfusion system. The inferior caval vein was cut to facilitate perfusion. The leg was flushed with carbogen-gassed Tyrode solution for 2.5 minutes (to 
remove blood cells) and subsequently with fixative ( $2.5 \%$ glutaralldehyde vol/vol in $0.1 \mathrm{M}$ phosphate buffer with $1.5 \%$ glucose $(\mathrm{D}+) . \mathrm{H}_{2} \mathrm{O}$ wt/vol added; $305 \mathrm{mOsm}$ without glutaraldehyde, $\mathrm{pH}=7.3$ ) for $9.5 \mathrm{~min}$. The limb was perfused at a pressure of $100 \mathrm{~mm} \mathrm{Hg}$. Perfusion fluids were prewarmed in a waterbath and had a temperature of $37^{\circ} \mathrm{C}$ when reaching the aortic cannula. The time interval between ligation of the abdominal aorta and perfusion with the fixative ranged from 4 to 11 minutes.

The perfusion flow during fixation was $12.8 \pm 2.6$ (SD) $\mathrm{ml} / \mathrm{min}(\mathrm{n}=12$ ) with virtually no change during the perfusion period and no clear differences between the four groups, indicating a constant resistance of the perfused vascular bed.

The fixed soleus muscle was dissected, and small tissue blocks were excised from the central part and postfixed in the glutaraldehyde fixative at $4^{\circ} \mathrm{C}$ for $24-48 \mathrm{~h}$. Subsequently, the tissue blocks were washed in phosphate buffer with glucose $\left(4^{\circ} \mathrm{C}, 15\right.$ min), postfixed in $\mathrm{OsO}_{4}$ in phosphate buffer ( $1 \%$ in phosphate buffer, $4^{\circ} \mathrm{C}, 60 \mathrm{~min}$ ), and washed again in phosphate buffer $\left(4^{\circ} \mathrm{C}, 15 \mathrm{~min}\right)$. Then the tissue was subsequently dehydrated through graded acetone $\left(50,70,80\right.$, and $90 \% ; 4^{\circ} \mathrm{C} ; 2 \mathrm{~min}$ each), followed by two rinses of acetone $100 \%$ (RT, 20 min each). Finally, the muscle tissue samples were transferred to propylene oxide ( $R T, 60 \mathrm{~min})$ and $50 \%$ propylene oxide/50\% Epon-DMP-30 (RT, overnight) and embedded in EponDMP-30.

\section{Light- and electron-microscopy}

From each muscle at least three randomly chosen blocks with embedded tissue were sectioned. Because the muscles of the rats in the 24-h group contained fibers that were invaded by monomorphonuclear leukocytes (see Figures. 3.1B and 3.2A), three sub-groups were defined in the $24 \mathrm{~h}$-group. The first subgroup consisted of capillaries adjacent to degenerated muscle fibers, the second subgroup consisted of capillaries radially about $50 \mu \mathrm{m}$ away from invaded muscle fibers, and the third subgroup consisted of capillaries radially $>250 \mu \mathrm{m}$ away from degenerated muscle fibers.

Semithin sections $(1 \mu \mathrm{m})$ were cut with glass knives and stained with $1 \%$ toluidine blue for light microscopy. Perpendicularly cut sections were used to search for tissue blocks with invaded muscle fibers (Figure 3.1) and collapsed or obstructed capillaries and to determine muscle fiber cross-sectional area and capillary density. Sections cut parallel to the muscle fibers were used to determine the sarcomere length.

For electron microscopy ultrathin sections were cut perpendicular to the muscle fiber orientation with a diamond knife on a microtome (Ultracut, Reichert-Jung, Austria). Sections showing a silver gray interference color were collected on a 75 mesh copper grid with collodion supporting film and subsequently stained with uranyl acetate ( $7 \%$ w/v in aqua dest; $10 \mathrm{~min}$ ) and basic lead citrate ( $5 \mathrm{~min})$. Deviation from the ideal angle $\left(90^{\circ}\right)$ between the sectioning plane and the mean muscle fiber orientation was $<5^{\circ}$. 
Compression due to semi- and ultrathin sectioning was assessed by comparing the dimensions of the section and the dimensions of the tissue block and was found to influence the measured parameters by $<1.5 \%$.

Sections were investigated with a Philips EM400 electron microscope for fixation quality, morphological characteristics of the muscle fibers, and the localization of morphologically abnormal capillaries. For each of the (sub)groups, 27 capillaries with a circular appearance were randomly selected. The entire capillary (Figure $3.2 \mathrm{~B}$ ) and a section without a nucleus (Figure 3.2C) were micrographed at $\times 2590$ and $x 6220$, respectively. The negative images were subsequently enlarged $7-12$ times and printed on photopaper. For calibration of the magnification a 1200 lines/mmgrid was used.

\section{Morphometry}

Video images of semithin sections and photographs of ultrathin sections were morphometrically analyzed with a Kontron Mop-Videoplan digitizer system (Zeiss, FRG).

The sarcomere length was measured from longitudinally cut semithin sections (45 muscle fibers/group). Perpendiculary cut semithin sections were used to count the number of capillaries around individual muscle fibers and to measure the median cross-sectional area of muscle fibers ( 16 blocks, 1 fiber/block, for the 24 -h invasive group and 9-12 blocks, 11-22 fibers/block, for each of the other groups).

The different capillary parameters, as determined from the micrographs, are presented in Figure 3.2D and Tabel 3.1. The "minimal diameter" of the capillary lumen was determined by taking the largest diameter perpendicular to the maximal diameter. A form factor, indicating the regularity of the capillary luminal contour, was calculated by dividing the measured cross-sectional surface area by that of the ellipse that theoretically, on the basis of inertia, fitted best to the drawn structure. The cross-sectional surface area of the endothelial cytoplasm was calculated by subtracting the nuclear area from the total endothelial area. The mean thickness of the capillary wall (cytoplasm of the endothelium) and of the capillary basal membrane were derived from five and three to five measurements, respectively. With the assumption that capillary interendothelial cleft width was constant, a relative measure of the transport resistance (TR) through the clefts was calculated from the individual inverted cleft length ( $L$ ) by use of the relation

$$
1 / \mathrm{TR}=1 / \mathrm{L} 1+1 / \mathrm{L} 2+\ldots+1 / \mathrm{Ln}
$$

\section{Relative soleus muscle water content}

Of five to seven rats of each group, the nonperfused soleus muscles was dissected immediately after anesthesia and quickly frozen in liquid nitrogen. The muscle was 
weighed on a microbalance (Satorius H51, Zeiss), freeze dried (GT2; Leybold-Heraeus) and weighed again. Relative muscle water content was callculated by dividing the weight loss due to the freeze drying by the fresh muscle weight.

\section{Statistics}

The values of rectal temperature, relative soleus muscle water content and capillary density appeared to have a normal distribution. These results are presented as means \pm SD and are evaluated for statistical significance by means of a two-sided Student's $t$ test. All other data are presented as median values and interquartile ranges (in parentheses), as in Tabel 3.1, or as a box plot, as in Figure 3.4, which in addition shows the minimal and maximal values. The Mann-Whitney $U$ test with correction for ties, was used for analysis of these parameters. Differences between groups were considered to be statistically significant when $P<0.05$.

\subsection{RESULTS}

\section{Degree of exhaustion of the animals}

Rectal temperature was $37.5 \pm 0.4^{\circ} \mathrm{C}(\mathrm{n}=18)$ at rest and $40.2 \pm 0.4^{\circ} \mathrm{C}(\mathrm{n}=15)$ immediately after the animals ran. Rectal temperatures returned to the control values within 1.5 hour after running. The exercise in the present study was most probably performed at submaximal intensity because 1) rectal temperatures never reached values attained at exhaustion $\left(41.8 \pm 0.3^{\circ} \mathrm{C}\right.$ ) (Gollnick and Ianuzzo, 1968), 2) the untrained rats were still able to continue running at the end of the exercise procedure, and 3) no glycogen depletion was found in any of the groups, as indicated by the presence of numerous glycogen granules in all muscle micrographs.

\section{Morphology of muscle fibers}

In general, the morphological features of the muscle fibers were similar to those described by Kuipers et al. (1983). Muscle fibers had a normal appearance in all groups, and mitochondrial disruption was rarely seen. The sarcomere length of the muscle fibers of the $\mathrm{C}$ group was $2.75 \pm 0.22$ (SD) $\mathrm{m}$, and no significant differences could be found between the different groups, indicating that the degree of contraction of the muscles during perfusion was about the same in all groups. Degenerated muscle fibers invaded by monomorphonuclear leukocytes were almost exclusively found 24 hours after exercise. They were always located between muscle fibers with a normal appearance (figure $3.1 \mathrm{~B}$ ). 

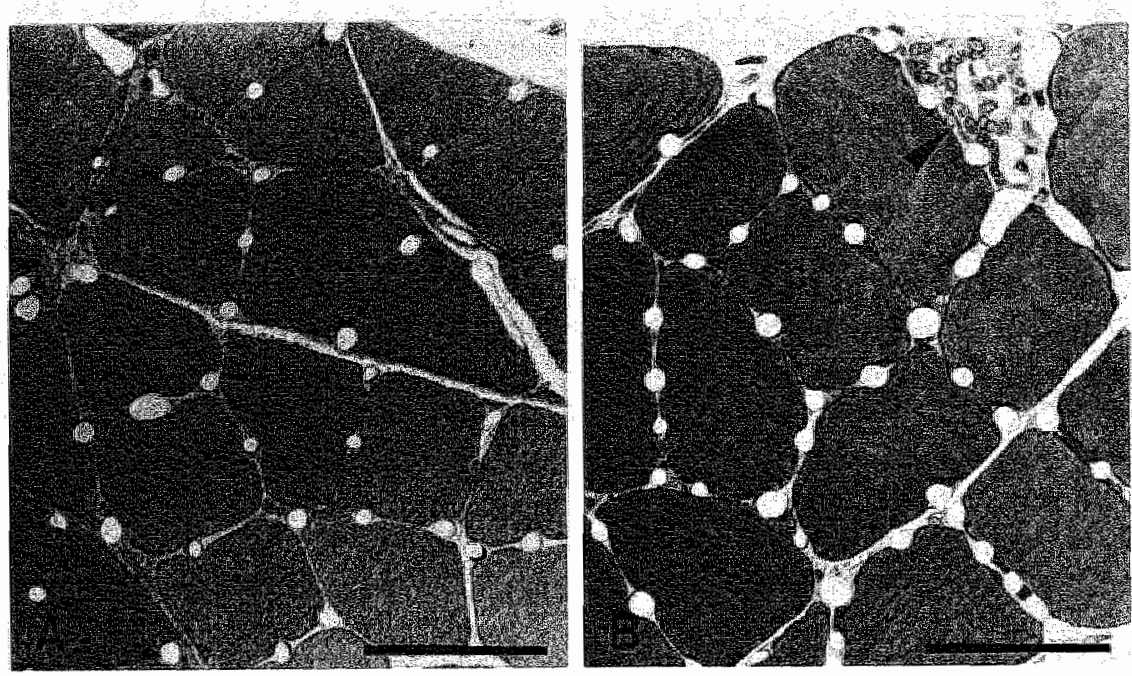

Fig. 3.1. Light-microscopic cross-sections of perfusion-fixed soleus muscle before (A) and 24 hours after endurance running (B). Arrow, muscle fiber invaded by monomorphonuclear leukocytes 24 hours after running. Bars, $50 \mu \mathrm{m}$.

Muscle fiber swelling and relative water content

The median cross-sectional area of the muscle fibers immediately or $24 \mathrm{~h}$ after the animals ran did not differ from control value $\left(1,352(1,157-1,636) \mu \mathrm{m}^{2} ; \mathrm{n}=9\right.$ blocks). However, $6 \mathrm{~h}$ after the animals ran, the cross-sectional fiber area was significantly increased by $28 \%\left(1,728(1,506-1,882) \mu \mathrm{m}^{2} ; \mathrm{P}<0.01 ; \mathrm{n}=9\right.$ blocks), indicating that the fibers were swollen. Surprisingly, soleus water content of the 6-h group $(76.5 \pm 1.5 \% ; n=8)$, as well as of all other experimental groups, did not differ significantly from the control value $(76.3 \pm 0.6 \% ; n=12)$.

\footnotetext{
Fig. 3.2. (Facing page) Soleus muscle 24 h after exercise. (A) micrograph of a muscle fiber invaded by monomorphonuclear leukocytes. Adjacent muscle fibers and capillaries have a normal appearance. Bar, $10 \mu \mathrm{m}$. (B) micrograph of capillary adjacent to an invaded muscle fiber. Bar, 1 um. (C) micrograph of a detail of cytoplasmic region of a capillary adjacent to an invaded muscle fiber. Bar, $0.5 \mu \mathrm{m}$. D: schematic drawing of a capillary with endothelial nucleus from control group based on median values. av, Abluminal vesicles; bm, basal membrane; $c v$, cytoplasmic vesicles; ec, endothelial cytoplasm; ic, interendithelial cleft; lu, lumen; Iv, luminal vesicles; $m$, mitochondrion; $n$, nucleus.
} 

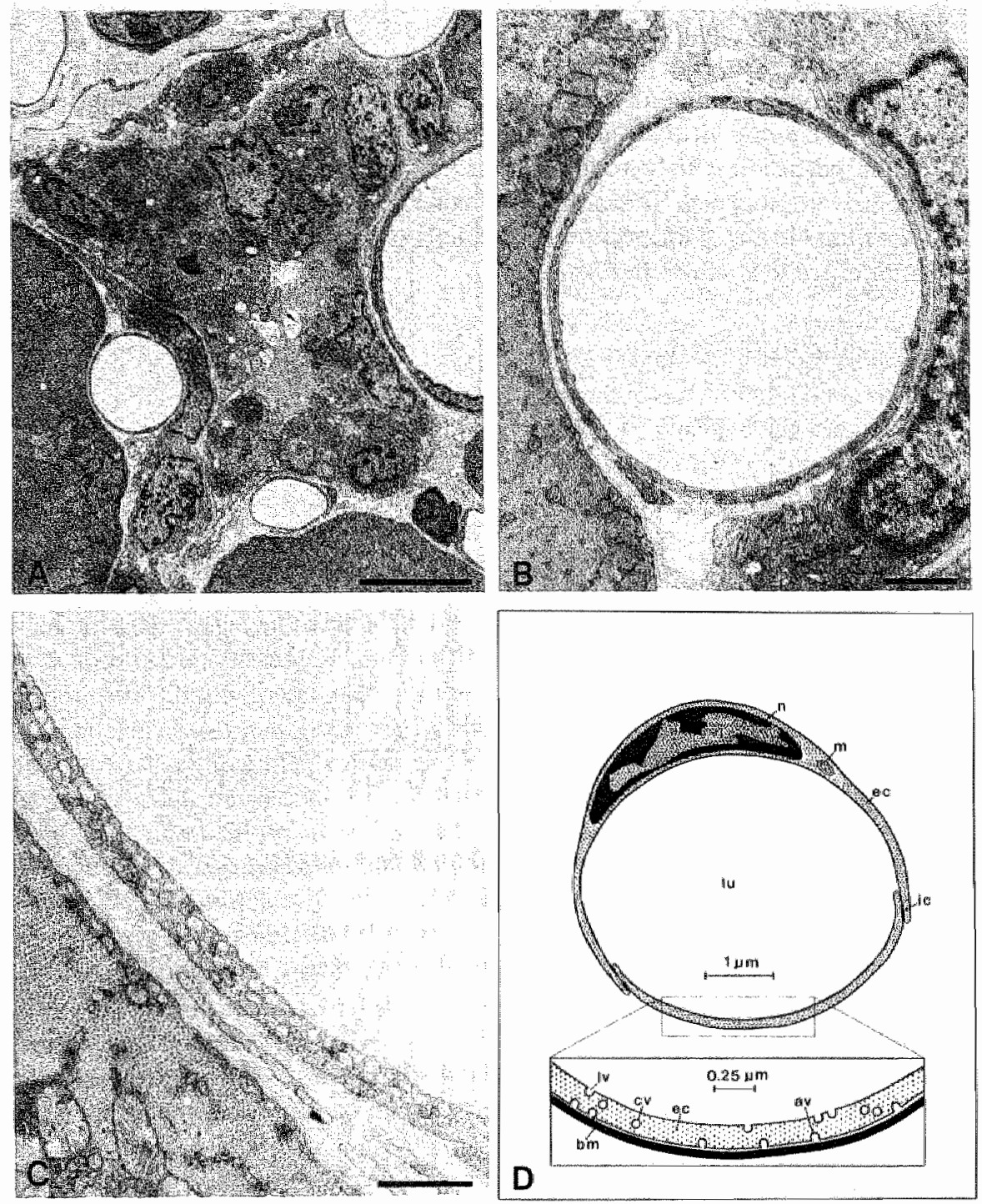


\section{Parameters indicative of capillary perfusion (Table 3.1)}

\section{Morphology of the capillaries:}

In general, capillaries of all groups generally had a normal appearance. Swollen endothelial cells, as found after ischemia (Gidlö et al., 1987B), could not be detected. In each group virtually all capillary humina were free of blood cells as a result of the perfusion with Tyrode solution and fixative. Collapsed or obstructed capillaries were not seen. Therefore focal concentrations of abnormal capillaries were not found in any of the groups. An example of soleus muscle tissue before and $24 \mathrm{~h}$ after exercise is shown in Figure 3.1.

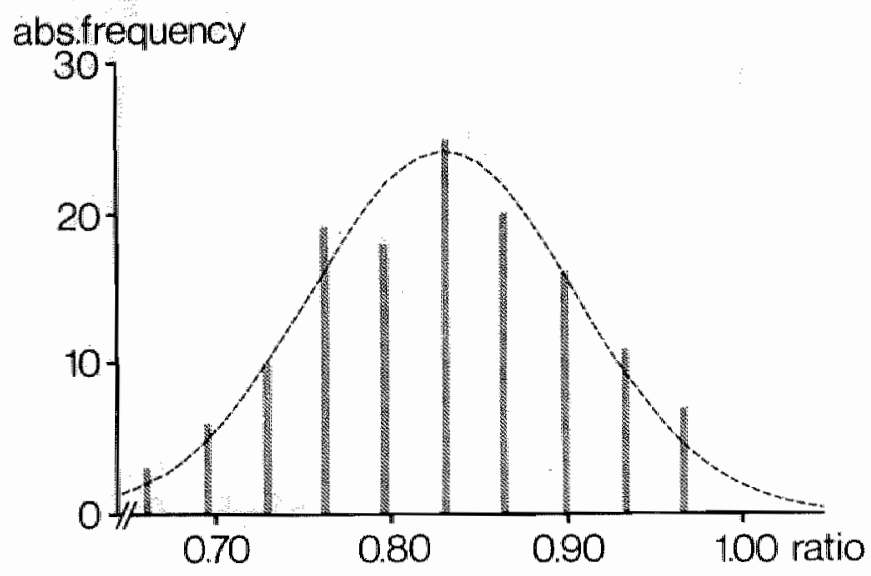

Fig. 3.3. Distribution of ratio between minimal and maximal capillary luminal diameters for 10 classes of equal widths. Total number of capillaries is 135. Bestfitted normal distribution (dashed line) corresponds well with the histogram, indicating that the capillaries are not round tubes (see text), abs, Absoiute.

\section{Capillary density:}

The mean number of capillaries around individual muscle fibers was $7.60 \pm 1.18$ ( $n=10$ blocks) in the $C$ group. No difference could be found between the groups (including the 24-h invasive group).

\section{Capillary lumen:}

The form factor of the capillary lumina of all groups did not significantly differ from 1, demonstrating that the luminal contours were regular. The capillaries of the soleus muscle were elliptical rather than circular. The ratio between minimal and maximal diameter of the capillary lumina was nórmally distributed around a mean value of $0.83 \pm 0.08$ (SD) $(n=135$; see Figure 3.3$)$. The long axis of the capillary humina was generally oriented parallel to the adjacent myocyte membrane. 


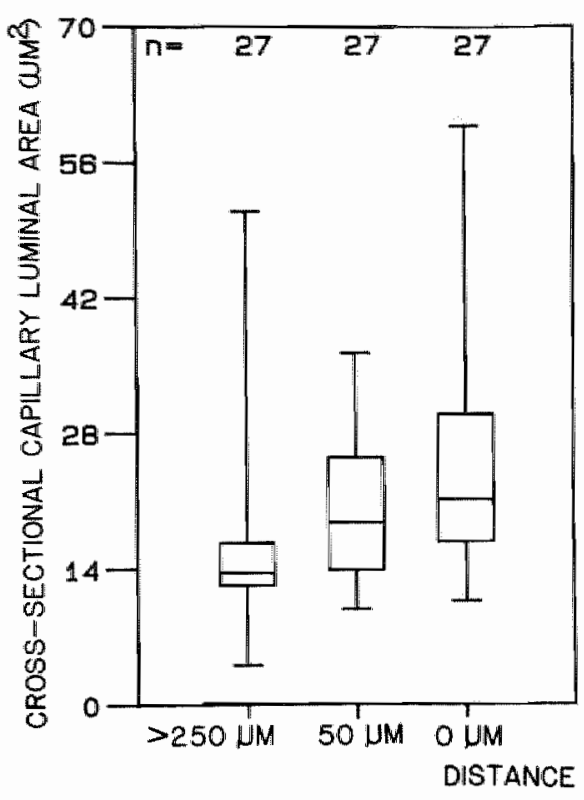

Fig. 3.4. Capillary luminal area 24 hours after running, as a function of distance $(\mu n)$ from invaded nuscle fibers. Box plots of mediain, interquartile range, and minimal and maximal values. $n$, No of capillaries. Results are significantly different for all (sub)groups $(P<0.05)$.

Capillaries adjacent to invaded muscle fibers (24-h invasive group) showed a significantly increased minimal luminal diameter (about $20 \%$ ) and cross-sectional area (about $50 \%$ ), as compared with all other groups. The increase in total capillary crosssectional area was mainly caused by an enlargement of the capillary lumen. Figure 3.4 shows box-plots of the cross-sectional luminal area of capillaries adjacent to, about $50 \mu \mathrm{m}$ or more than $250 \mu \mathrm{m}$ away from the invaded fiber area. The values of capillaries about $50 \mu \mathrm{m}$ away from the invaded area were intermediate between and significantly different from that of capillaries $>250 \mu \mathrm{m}$ from the invaded fibers and of the capillaries adjacent to invaded fibers. Figure 3.5 shows that the cumulative distribution of luminal cross-sectional areas of capillaries $>250 \mu \mathrm{m}$ away from invaded muscle fibers did not differ from the distribution of control values. On the other hand, the cumulative distribution of the values for capillaries adjacent to invaded muscle fibers was shifted towards larger values, indicating a capillary dilatation independent of the initial diameter.

\section{Parameters indicative of capillary exchange (Table 3.1)}

\section{Morphology of the endothelium:}

In general, the endothelial organelles of all groups had a normal appearance. No interendothelial gaps, i.e., local detachments of cellular contact, could be found in any of the groups. 


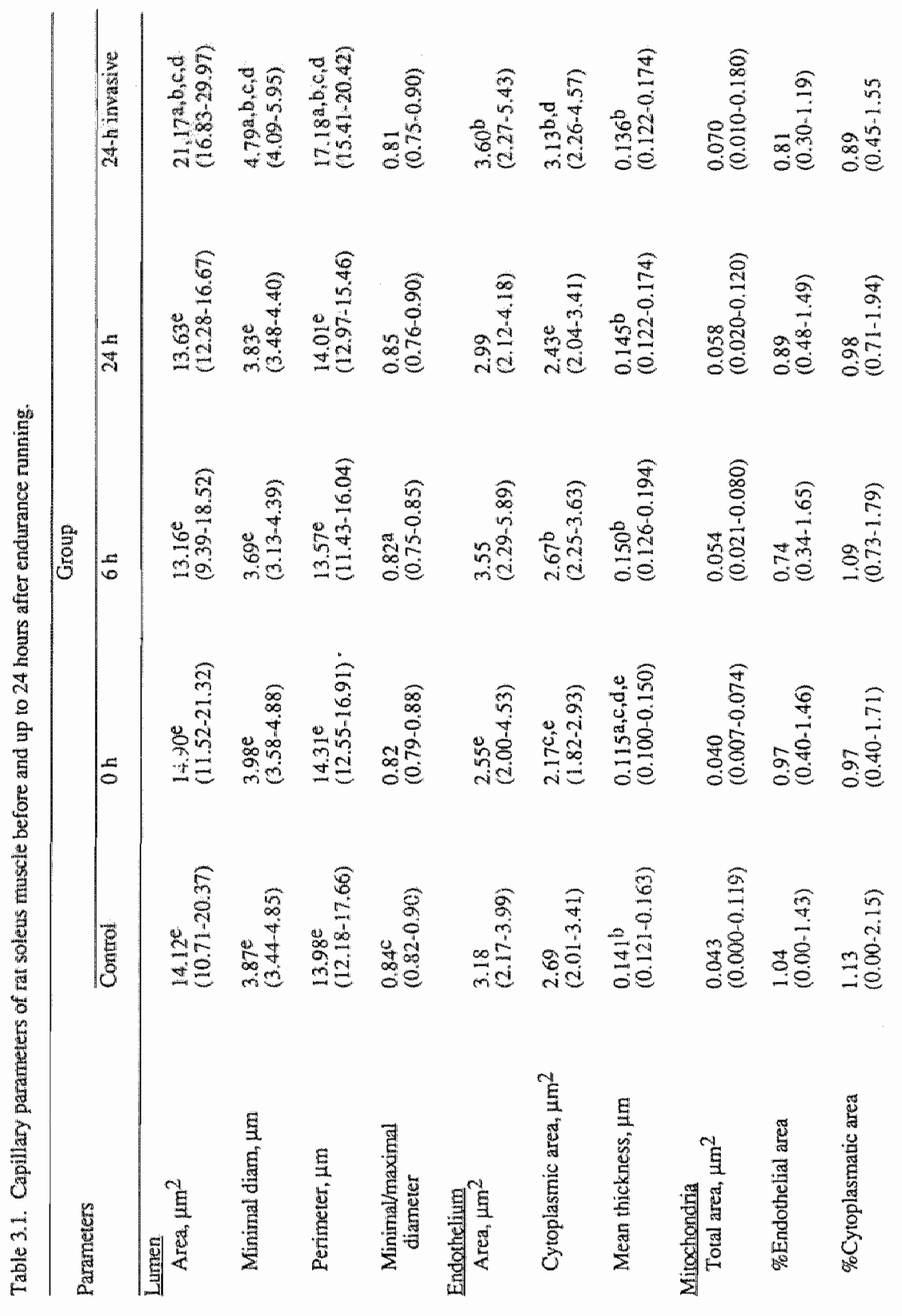




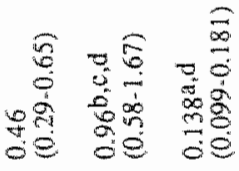

\&

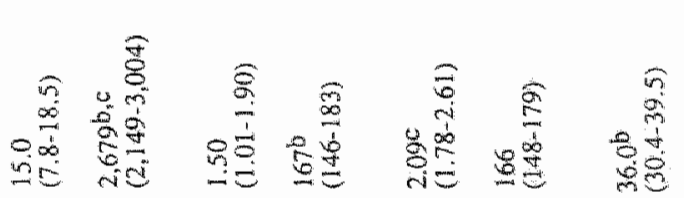

3
5

vi

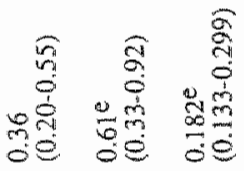

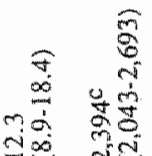

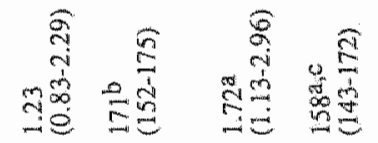

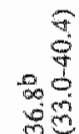

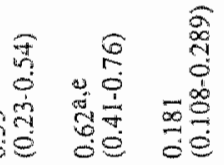

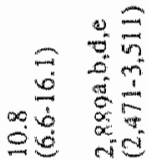

$\begin{array}{rl}3 & 0 \\ 0 & 0 \\ 4 & 0 \\ 0 & 0 \\ 0 & 0 \\ 0 & 0\end{array}$

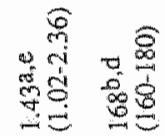

$\frac{b}{a+j}$

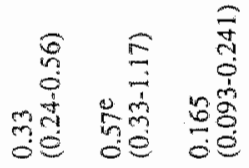

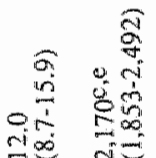

ले प्ष

\% 8 के

起

है

की.

त.

$\overbrace{11}^{2} \frac{5}{8}$

II ते

हैं

放

$\frac{2}{4}$

30

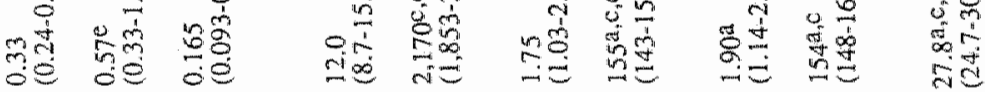

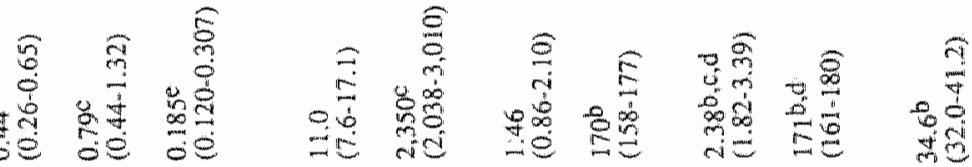

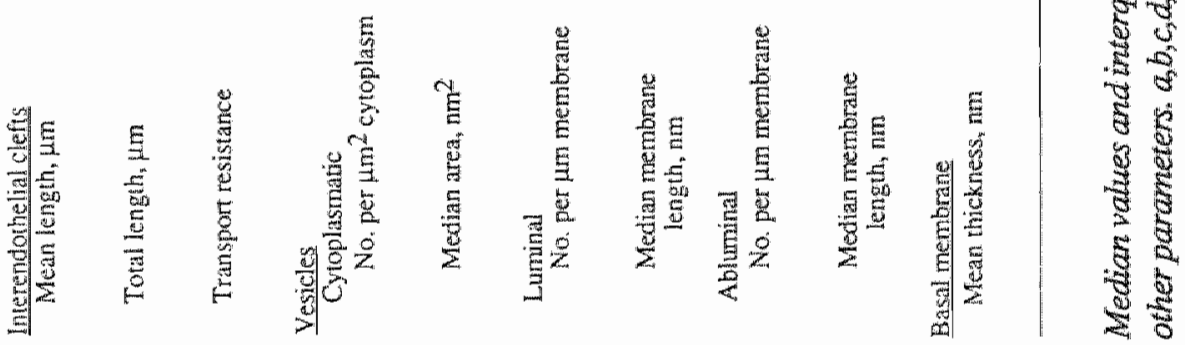




\section{Capillary lumen:}

The increased luminal cross-sectional area of the $24 \mathrm{~h}$-invasive group resulted in an increased length of the luminal endothelial membrane, which is indicative of an increased capillary exchange area.

Capillary wall thickness:

The thickness of the endothelial cytoplasm and basal membrane was about $20 \%$ smaller in the 0-h group than in the other groups.

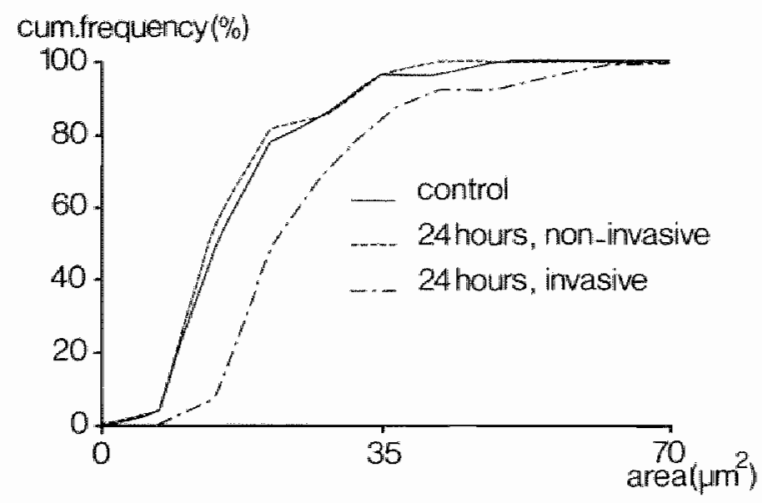

Fig. 3.5. Capillary luminal area before (control) and $24 \mathrm{~h}$ after running. Cumulative distribution of capillary cross-sectional luminal area. Twenty-four hours after running capillaries $>250$ um away from invaded muscle fibers (24 hours, non-invasive) show a distribution similar to that in the control group. Capillaries adjacent to invaded muscle fibers (24 hours, invasive) show a general increase of their luminal areas, independent of their initial size.

\section{Vesicles:}

The median cross-sectional area of the cytoplasmic vesicles in the capillary endothelium was significantly higher in the 6-h group, compared with all other groups. The membrane length of the luminal vesicles of the capillary endothelium was slightly, but significantly, smaller in the 0-h group than in the other groups. The number of abluminal vesicles per micrometers abluminal membrane was significantly reduced by about $30 \%$ in the $0-, 6-$, and 24 -h groups, compared with the C group. In none of the groups was the entire vesicular system changed as a result of the exercise procedures.

In each group ( 27 capillary segments per group), three to five transendothelial channels were found.

Other parameters:

No major differences between the groups were found for any of the mitochondrial and interendothelial cleft parameters studied. 
About $34 \%$ of all capillaries investigated showed an endothelial nucleus. No marked differences in percentage of capillaries with endothelial nucleus were found between the groups.

\subsection{DISCUSSION}

To study a possible microcirculatory involvement in focal muscle fiber degeneration after exercise, we investigated whether changes in the capillary morphology in rat soleus muscle could be detected after submaximal endurance running. The mophological and morphometric findings do not support the hypothesis that, in the first 24 hours after endurance exercise, obstructed capillary blood flow and/or impaired exchange capacity cause the focal degeneration. Around exercise-induced degenerated muscle fibers, capillaries are dilated $24 \mathrm{~h}$ after exercise.

Uphill running, inducing moderate fiber degeneration with a focal character (Kuipers et al., 1983; present study), allowed us to study morphological changes in capillaries adjacent to degenerated fibers and in capillaries at a certain distance from these fibers. The median cross-sectional luminal area of the capillaries adjacent to degenerated muscle fibers (24-h invasive group) was increased, compared with the capillaries in the other groups. This phenomenon decreased with increasing distance from the invaded muscle fibers, suggesting that the changes are related. to local factors. The general increase of skeletal muscle capillary luminal cross-sectional area in several inflammatory myopathies in humans (Jerusalem et al., 1974B; Hoppeler, 1986) and the resemblance of the muscle fiber degeneration in these myopathies and those found in our study suggests that similar, as yet unknown, underlying mechanisms are involved.

It is unlikely that the muscle fiber damage, as found 24 hours after exercise, is induced by factors other than running. Kuipers et al. (1983) reported that the periodical electrical current on the resting grid used in this experiment is too low to cause muscle fiber damage. In addition, the time period between ligation of the abdominal aorta and perfusion fixation of the soleus muscle is too short (maximally $11 \mathrm{~min}$ ) to cause ischemic ultrastructural changes of either muscle fibers (Maikitie and Teräväinen, 1977) or capillaries (Mäkitie, 1977).

The morphometric data could have been influenced by shrinkage of the muscle or by distortion or tortuosity of capillaries. Because the soleus muscles were fixed and embedded at the same relative length, as was shown by their similar sarcomere length after processing, shrinkage, if any, because of this procedure is likely to be similar for all groups. Therefore, in this respect, the data in the present study may be compared directly. Distortion of the capillaries, as a result of compression due to cutting, electron-microscopic imaging, or projection of the negative on the photopaper and the morphometer was found to be negligible. Although the cross-sections were perpendicular to the muscle fiber orientation and capillaries with the most circular lumina were selected, the ratio between the minimal and maximal capillary 
Iuminal diameter was normally distributed around a value significantly $<1$ (mean $=0.83$; see Figure 3.3), as would be expected for meandering elliptical tubes. For meandering circular tubes, a skewly distributed ratio with a maximal value at 1 would be expected. Therefore, the capillaries in our study must be ovally shaped.

If microcirculatory changes are related to focal muscle fiber degeneration, focal changes in capillary morphology, resulting in decreasing blood flow or trans capillary exchange; might lead to degeneration of the adjacent fiber(s). Capillary changes throughout the muscle might induce focal degeneration of the most vulnerable fibers. Although no morphometric data on muscle fibers or capillaries were obtained during the running period, it is unlikely that substantial morphometric changes durring running would be absent immediately thereafter (0-h group). However, a functional discrepancy between blood flow and demand during running cannot be excluded from the present data.

The unchanged number of capillaries around individual muscle fibers in any of the groups investigated indicates that no obvious loss of capillaries or angiogenesis occurs during the 24 hours after exercise. The absence of major changes in capillary morphology and of capillary obstructions in any of the groups suggests that changes in capillary morphology are not the major cause of exercise-induced focal muscle fiber degeneration. This view is supported by the finding that capillay morphology of human skeletal muscle is relatively unaffected after marathon running (Warhol et al. 1985). A microcirculatory contribution, however, cannot be excluded, because the muscle fiber swelling found at 6 hours after running may have led to an elevated tissue pressure and, hence, increased venous pressure and reduced perfusion pressure (Reneman et al., 1980; Tangelder et al., 1984). It is unknown whether this swelling actually raises tissue pressure, resulting in temporary impaired blood flow and subsequent muscle fiber degeneration.

Considerable muscle swelling was found $6 \mathrm{~h}$ after running ( $28 \%$ increase compared with control), in the absence of an increase in muscle water content. An increase in muscle water content was expected, because, in case of a dry mass of $24 \%$ in control muscle, a swelling of $28 \%$ would indicate an increase in muscle water content from $76 \%$ to about $(104 / 128) \times 100 \%=81 \%$. To exclude that the muscle fiber swelling, as seen on semithin sections, is due to fixation artifacts, we repeated the experiments and determined both water content and cross-sectional fiber area in soleus muscle that was cryofixed at a standard length. In these experiments water content was again unchanged $6 \mathrm{~h}$ after running, compared with control (3 rats/group), whereas the median cross-sectional muscle fiber area was again significantly increased by $26 \%$ ( 3 rats/group; 200 fibers/rat). Based upon these experiments, it may be concluded that the fiber swelling is not a fixation artifact but of a true nature. The discrepancy between fiber swelling, as found in muscle sections, and the absence of an increase in muscle water content is incompletely understood.

The absence of major changes in capillaries and capillary subcellular structures is indicative of the absence of a morphological basis for impairment of capillary exchange capacity, if any, under these circumstances. 
Immediately after running ( 0 -h group), we observed a transient reduction of the capillary thickness and basal membrane by $20 \%$. Because the cross-sectional area of the muscle fibers is hardly reduced, the reduction might have been caused by a selective exercise-induced dehydratation of the capillaries. Because the length of the luminal membrane is not reduced, the diminished endothelial thickness may have had a positive effect on the diffusion rate of substances between blood and muscle fibers. The decreased thickness of the capillary basal membrane might have facilitated the diffusion of macromolecules. A thinner endothelial cell layer might facilitate the formation of more transendothelial channels, but the number of channels observed are too low to draw conclusions at this point.

The increased cross-sectional muscle fiber area $6 \mathrm{~h}$ after running might reduce the diffusion rate of substances between muscle fibers and capillaries. It seems unlikely, however, that a small increase of diffusion distance (about $10 \%$ ), some hours after the actual exercise will cause muscle fiber degeneration several hours later.

Immediately, 6 , and $24 \mathrm{~h}$ after running, all capillaries showed a reduction of about $30 \%$ of the number of abluminal vesicles per unit membrane length, compared with the control group. If endothelial vesicles are involved in the exchange of macromolecular substances between blood and muscle fibers, as has been suggested by several investigators (Simiunescu et al., 1972 and 1973 and 1975; Pietra et al., 1982; Orgnero de Gaisan et al., 1985), this reduction might indicate that the exchange capacity for macromolecules is impaired at the abluminal side of the capillaries. Because in none of the groups the entire vesicular system is affected, the physiological. significance of these changes remains unclear.

Because the transport of ions, small molecules (Casley-Smith, 1980 and 1981), and even macromolecules (Schneeberger and Karnovsky, 1971; Orgnero de Gaisan et al., 1985) occurs mainly through interendothelial clefts, the relative transport resistance of the clefts was calculated. Neither the cleft resistance nor any of the other endothelial cleft parameters changed in any of the groups. Therefore, it seems unlikely that overall transport of substances through interendothelial clefts is substantially altered as a result of running.

Although no functional parameters were measured, one might speculate about the physiological consequence of some of the morphological findings. The ellipsoid cross-section of the capillaries found in this study could represent a "dimensional reserve" to be utilized for conformational changes during muscle contraction, increased perfusion or blood cell passage as has been suggested by Gidlöf $e t a l$. (1987A). The increased lumen of capillaries adjacent to degenerated and leukocyteinvaded muscle fibers results in decreased resistance and, hence, increased capillary blood flow. Because erythrocytes and leukocytes preferentially follow capillaries with the highest flow rate (Schmid-Schönbein, 1980; Blixt et al., 1987), these cells may be preferentially shunted to degenerated muscle fibers. The increased length of the luminal membrane of these capillaries results in an increased surface area for exchange of substances and leukocyte diapedesis, if any, through the capillary wall. These factors might facilitate muscle fiber repair. 
In conclusion, the anatomical findings on capillaries of rat soleus muscle before and up to 24 hours after an acute bout of exercise do nót indicate that exercise induced focal muscle fiber degeneration is primarily caused by capillary alterations. The anatomical findings are not in favour of an obstructed capillary flow or impaired exchange capacity. The focal necrosis and leukocyte invasion of the muscle fibers cause dilatation of nearby capillaries.

\subsection{SUMMARY}

The relationship between exercise-induced focal muscle fiber degeneration and changes in capillary morphology was investigated in male Wistar rats. Untrained animals ran on a treadmill for 1 hour at submaximal intensity and were killed 0,6 or 24 hours after running. Non-exercised rats served as controls. In situ perfused soleus muscles were prepared for electron microscopy. Micrographed cross-sections were quantitatively analysed for parameters indicative of capillary blood flow or transcapillary exchange. Capillary lumina were ovally rather than circularly shaped, and no indications for obstruction of blood flow at the capillary level were found. Enclothelial cells and their organelles had a normal appearance in all groups. However, immediately after exercise, capillaries showed a decreased thickness of their endothelium and basal membrane, probably caused by dehydration. Six hours after exercise, muscle fibers were swollen ( $28 \%$ increase in cross-sectional area), resulting in a slightly increased diffusion distance. This fiber swelling was not associated with an increase in muscle water content, a finding for which no explanation could be found. Twenty-four hours after the animals ran, capillaries located near degenerated muscle fibers had an increased cross-sectional luminal area and an increased luminal circumference. This effect decreased gradually with increasing distance from the degenerated fiber area.

The present morphometric results do not support the hypothesis that changes in capillary morphology primarily contribute to exercise-induced focal muscle fiber degeneration. 


\section{CAPILLARY (ULTRA)STRUCTURE IN RELATION TO POSITION AND FIBER TYPE IN RAT SOLEUS MUSCLE}

\subsection{INTRODUCTION}

Exercise can induce focal muscle fiber degeneration in rat soleus muscle (Kuipers et al., 1983; Peeze Binkhorst et al., 1989A). Although the soleus muscle contains both type I and type II fibers (Bennett et al., 1959; Brook and Kaiser, 1970; Pullen, 1977), the exercise induced degenerated fibers are exclusively of type I (Kuipers et al., 1983). In a previous study it was found that the lumen of capillaries adjacent to degenerated muscle fibers have an approximately $50 \%$ larger cross-sectional area than lumina of other capillaries (Peeze Binkhorst et al., 1989A). The larger lumen of capillaries around degenerated type I fibers might indicate the existence of capillary dilation due to inflamatory response. On the other hand, capillaries adjacent to type Ifibers might have larger lumina than capillaries adjacent to type II fibers, resulting in larger capillary lumina around degenerated (type I) fibers.

To the best of our knowledge, no data are available concerning the relation between capillary dimensions and muscle fiber type, probably because preservation of good capillary ultrastructure and histochemical typing of tissue require incompatible procedures. Therefore, a retrieval method, which combines muscle fiber typing with the preservation of muscle fiber and capillary (ultra)structure, was used. This method consisted of perfusion fixation of rat soleus muscles with paraformaldehyde fixative. Parts of the muscle were cut in half perpendicularly to the muscle fiber orientation. One half was embedded in paraffin, allowing fiber typing by using a monoclonal antibody (RIID10) ("fiber typing sections"). The counterpart was embedded in plastic (Epon) for morphometric analysis of fiber and capillary (ultra)structure ("morphometry sections"). Fibers in the morphometry sections were matched with their counterpart in the fiber typing sections (retrieval method). Using this methode we investigated whether capillaries supplying type I and capil- 
laries supplying type II fibers in soleus muscle differed morphologically. Special attention was payed to morphological aspects possibly of importance regarding capillary flow and/or exchange of substances between blood and muscle fiber.

\subsection{MATERIALS AND METHODS}

\section{Animals}

Male Brown Norwegian rats $(n=14), 10-11$ weeks old and weighing 230-260g, were used. The animals were housed in a temperature controlled environment (23$26^{\circ} \mathrm{C}$ ), and fed with rat chow (type 1210, Hope Farms), and water ad libitum.

The animals were anesthetized with sodium-pentobarbital (Narcovet; $60 \%$ solution; $1 \mathrm{ml} / \mathrm{kg}$ body weight, ip) prior to further handling.

Fixation of muscle tissue

For comparison of fiber staining for myosin ATPase activity and fiber immunolabeling, cryofixed rat soleus muscles were used. All muscles were freshly dissected and frozen in isopentane cooled down to $-157^{\circ} \mathrm{C}$ by liquid nitrogen.

To test the reactivity of the RIIDIO-antibody in the presence of chemical fixatives, freshly dissected rat soleus muscles were chemically fixed by immersion $\left(4^{\circ} \mathrm{C}\right.$, overnight), and subsequently cryofixed (see above). Chemical fixatives tested in this study were:

1. Paraformaldehyde (PF; $4 \% \mathrm{PF}$ wt/vol in $0.1 \mathrm{M}$ phosphate buffer with $1.5 \% \mathrm{glu}-$ cose $(\mathrm{D}+) . \mathrm{H}_{2} \mathrm{O}$ wt/vol, $305 \mathrm{mOsm}$ without the fixing agent, $\mathrm{pH}=7.3$ ).

2. Glutaraldehyde ( $\mathrm{GA}$; similar to PF-fixative but with $2.5 \% \mathrm{GA}$ vol/vol instead of $\mathrm{PF}$ ).

3. Paraformaldehyde and glutaraldehyde (PF/GA; similar to PF-fixative but with additional $0.5 \% \mathrm{GA} \mathrm{vol} / \mathrm{vol}$ ).

To preserve muscle (ultra)structure, the vascular bed of hindlimbs of the rats were chemically fixed by perfusion in situ. As a fixative PF was used, because this was the only fixative that preserved fiber type immunoreactivity and, at the same time, muscle ultrastructure (see results).

After anesthesia and median laparotomy, heparin (1,000 IU/kg body wt) was injected into the liver to prevent blood coagulation. Meanwhile, sodium-pentobarbital $(60 \%, 0.3 \mathrm{ml} / \mathrm{kg}$ body wt) was dripped into the abdominal cavity for additional anesthesia. Five minutes after heparin injection, the abdominal aorta was ligated just distal to the renal arteries.

For perfusion of the left hindlimb, a silicone cannula (602-155; Silastic; Dow Corning, USA) was inserted into the abdominal aorta and fixed with the tip positioned 
a few millimeters from the iliac bifurcation. The left hindlimb of each rat was fixed in a standard position (ankle $60^{\circ}$, knee $90^{\circ}$, hip $90^{\circ}$ ). The right iliac artery and vein were clamped and the aortic cannula was connected to the perfusion system. To fam cilitate perfusion, the inferior caval vein was cut. To remove blood, during 2 minutes the hindlimb was initially flushed during 2 minutes with $0.1 \mathrm{M}$ phosphate buffer $(\mathrm{pH}=7.3)$ supplemented with $1.5 \%$ glucose $(\mathrm{D}+) \cdot \mathrm{H}_{2} \mathrm{O}$ wt/vol and $2.5 \%$ bovine serum albumine $(100 \mathrm{~mm} \mathrm{Hg})$. Subsequently, the hindlimb was perfused at a continuous pressure of $13.3 \mathrm{kP}$ ( $100 \mathrm{~mm} \mathrm{Hg}$ ) for 10 minutes with the PF fixative. Perfusates were prewarmed in a waterbath and entered the abdominal aorta at $37^{\circ} \mathrm{C}$. Time between ligation of the abdominal aorta and perfusion with the fixative ranged from 5-13 minutes.

The fixed soleus muscle was dissected. Small samples of the central part were excised and postfixed in PF fixative for $24-48 \mathrm{~h}$ at $4^{\circ} \mathrm{C}$. Subsequently, these muscle samples were washed in phosphate buffer with $1.5 \%$ glucose $(\mathrm{pH}=7.3,305 \mathrm{mOsm}$, $4^{\circ} \mathrm{C}, 15 \mathrm{~min}$ ), and carefully cut into two halves perpendicularly to the mean muscle fiber orientation.

One half of each muscle sample was dehydrated through graded ethanol $(70 \%$, $80 \%, 90 \%, 96 \%, 2$ times $100 \%$; room temperature (RT); 30 min each) and histoclear (RT; $2 \times 60 \mathrm{~min}, 1 \times$ overnight), and embedded in paraffin (max. temperature $60^{\circ} \mathrm{C}$ ). This part was used for immunohistochemical fiber typing. Its counterpart muscle sample was postfixed in $1 \% \mathrm{OsO}_{4}$ in phosphate buffer $\left(4^{\circ} \mathrm{C}, 60 \mathrm{~min}\right)$, and washed three times in phosphate buffer $\left(4^{\circ} \mathrm{C}, 15 \mathrm{~min}\right)$. This part was then dehydrated through graded ethanol $\left(50 \%, 70 \%, 80 \%, 90 \% ; 4^{\circ} \mathrm{C}, 5\right.$ min each), rinsed two times in ethanol $100 \%\left(4^{\circ} \mathrm{C}, 30 \mathrm{~min}\right.$ each), transferred into propylene oxide $\left(4^{\circ} \mathrm{C}\right.$, two rinses, $60 \mathrm{~min}$ ), $50 \%$ propylene oxide $/ 50 \%$ Epon-DMP-30 (RT, two rinses, 60 $\mathrm{min}$ ), and subsequently rinsed (RT, two times, $60 \mathrm{~min}$ ), and embedded in EponDMP-30. This part was used for analysis of capillary and fiber (ultra)structure.

\section{Identification of fiber types}

\section{Histochemical identification of fiber types:}

Cryosections $(10 \mu \mathrm{m})$ of freshly cryofixed muscles were cut perpendicularly to the average muscle fiber orientation with a cryostat at $-20^{\circ} \mathrm{C}$ and stained for myofibrillar myosin ATPase activity. Reactions were conducted at pH $9.4\left(30 \mathrm{~min}, 37^{\circ} \mathrm{C}\right)$ after preincubation at $\mathrm{pH} 4.3\left(9 \mathrm{~min}, 37^{\circ} \mathrm{C}\right)$, allowing differentiation between type I, type IIA/IIB and type IIC fibers (Brooke and Kaiser, 1970).

Immunohistochemical identification of fiber types:

The monoclonal anti-cardiac myosin IgG (R11D10; Centocor, USA) (Khaw et al., 1984), which reacts in skeletal muscles with type I muscle fibers, but not with type II muscle fibers (Havenith et al., 1989; this study), was used for immunohistochemical fiber typing. The specificity of the antibody was tested by comparing immunohistochemically stained, unfixed cryosections with consecutive cryosections stained for myosin ATPase activity (Dubowitz, 1985). 
Direct labeling of type If fibers on sections of Epon embedded PF-fixed soleus muscle, using a conventional $10 \mathrm{~nm}$ gold probe coupled to protein-A and omitting the $\mathrm{OsO}_{4}$ in the fixation procedure, failed. Therefore, an indirect identification method was developed. RIID10-labeled sections of paraffin embedded muscle were used to identify type I and type II soleus muscle fibers in sections of their Epon embedded counterparts (see results).

For immunolabeling, paraffin embedded sections $(5 \mu \mathrm{m})$ of muscle tissue were cut perpendicularly to the mean musele fiber orientation, dewaxed in xylol and transferred to $100 \%$ and $96 \%$ ethanol, respectively. These sections, as well as $10 \mu \mathrm{m}$ sections of frozen tissue (unfixed, PF, PF/GA or GA fixed) were incubated in $0.5 \%$ $\mathrm{H}_{2} \mathrm{O}_{2}$ in methanol (15 min, RT), $0.1 \%$ pepsin (Sigma) in $0.1 \mathrm{M} \mathrm{HCl}\left(30 \mathrm{~min}, 37^{\circ} \mathrm{C}\right.$; except cryosections). Then the tissue was washed in aqua dest and Tris buffer $(0.1 \mathrm{M}$, $\mathrm{pH}=7.6, \mathrm{RT}$ ), respectively. Subsequently, the sections were incubated in normal non-immune rabbit serum (diluted in Tris buffer, $10 \mathrm{~min}, \mathrm{RT}$ ) to reduce nonspeciffic background staining. The sections were then incubated in a moist chamber ( 45 min, RT) with the monoclonal anti-cardiac myosin IgG (RI1D10), diluted 1:2,000 in Tris buffer/bovine serum albumin (BSA), and washed three times in Tris buffer ( $5 \mathrm{~min}, \mathrm{RT}$ ). Subsequently, sections were incubated with peroxidase-labeled rabbit anti-mouse IgG antibodies diluted 1:200 in Tris buffer/BSA (45 min, RT), and rinsed three times in Tris buffer ( $5 \mathrm{~min}, \mathrm{RT}$ ). The sections were then incubated in $0.01 \% \mathrm{H}_{2} \mathrm{O}_{2}, 0.05 \%$ diaminobenzidine tetrahydrochloride and $10 \%$ imidazol in Tris buffer ( 5 min, RT). Finally, haematoxylin was applied ( 5 min., RT) for nuclear staining. The immunostained sections were used for typing of fibers in sections used for morphometry and to determine fiber type clustering.

\section{Light- and electronmicroscopy of Epon-embedded sections}

Semithin sections $(1 \mu \mathrm{m})$ of Epon embedded muscle were cut perpendicular to the muscle fiber orientation with glass knives, stained with $1 \%$ toluidine blue and investigated under a light microscope (objective 40x; numerical aperture 0.65 ). These sections were used to determine cross-sectional areas of type I and type II muscle fibers. Because no collapsed capillaries were found, light microscopy could be used to identify capillaries and determine their number around individual muscle fibers.

For electron microscopy ultrathin sections were cut perpendicular to the muscle fiber orientation with a diamond knife on a microtome (Ultracut, Reichert-Jung, Austria). Ultra-thin sections showing a silver gray interference color were collected on a 75 mesh copper grid with collodion supporting film and subsequently stained with uranyl acetate ( $7 \%$ wt/vol in aqua dest; $10 \mathrm{~min}$ ), and basic lead citrate (5 min).Fixation quality and cellular morphology were investigated using a Philips EM400 or a Philips CM12 electron microscope.

Because the number of type I fibers was about 4 times as high as that of type II fibers (see results), the chance of finding a capillary situated between more than 
two type I fibers was much larger than finding a capillary situated between more than two type II fibers. Therefore, if capillaries are selected at random, differences; if any, between capillaries situated between type I or between type II fibers might be related to the adjacent fiber type, or to the number of surrounding fibers. To check gross differences between capillaries situated between two or between more than two fibers, we compared luminal dimensions of these capillaries (72-98 capillaries per rat, 7 rats), using a relatively quick method of morphometric analysis of video recordings of STEM (scanning transmission electron microscopy; size of scanning spot $15 \mathrm{~nm}$ ) images (magnification 4830x). Capillaries that could not be classified into one of both groups were rare.

For a more detailed analysis of capillary morphology, conventional transmission electron microscopic micrographs were used. To distinguish between capillaries related to type I and type II fibers in soleus muscle, the two most extreme situations were selected, being capillaries situated exclusively between type I (IIr) muscle fibers and capillaries situated exclusively between type II (tIIr) muscle fibers. Capillaries between type I and type II fibers were considered to be intermediate and were not included in the analysis. Capillaries of either type were randomly selected. However, capillaries situated between more than two fibers were rejected because they differed morphometrically from capillaries situated between two fibers (see results). Capillaries situated between more than two type II fibers were rare. Because the morphometry of the capillaries depends in part on the angle at which they are cut, for practical reasons, capillaries with extremely oval lumina (maximal/minimal diameter $>1.5$ ) were also excluded for further study. Eventually, from each rat $(\mathrm{n}=7)$ 4-7 capillaries of either type (tIr and tIIr) were selected.

An entire capillary cross-section (Figure 4.1A), and about one fourth of the capillary without a nucleus (Figure 4.1B), were micrographed at a magnification of $2590 \mathrm{x}$ and $8850 \mathrm{x}$, respectively. The negative images were subsequently enlarged 7 times and printed on photopaper. For calibration of the magnification of the electronmicroscopic immages, a 2160 lines $/ \mathrm{mm}$ grid was used.

\section{Morphometry}

With the technique used, all muscles were fixed at the same relative length and the influence of compression on the measured parameters was less then $1.5 \%$ (Peeze Binkhorst et al., 1989A). Tissue shrinkage due to processing was considered to be the same in all plastic embedded (morphometric) preparations.

Video images of semithin and ultrathin Epon embedded sections and photographs of ultrathin sections were morphometrically analyzed with a Kontron MopVideoplan digitizer system (Zeiss, FRG).

From semithin Epon embedded sections the cross-sectional area of muscle fibers was measured, the mean value per rat calculated and the number of capillaries around each muscle fiber counted (20 muscle fibers per type per rat; 10 rats). 
The different capillary parameters, as determined from the micrographs, are presented in Table 4,2. The "minimal diameter" of the capillary lumen was determined by taking the largest diameter perpendicular to the maximal diameter. The area of the endothelial cytoplasm was calculated by subtracting the nuclear area from the total endothelial area. The mean thickness of the capillary wall (cytoplasm of the endothelium) was calculated by dividing the endothelial cytoplasmic area of a capillary (endothelial) cross-sectional segment (not including the nucleus) by the length of the same segment $(2.2-3.9 \mathrm{\mu m})$. The mean thickness of the capillary basal membrane was calculated accordingly. Assuming capillary interendothelial cleft width to be constant, an approximate relative measure for the transport resistance (TR) of substances through the clefts was calculated (Peeze Binkhorstet al., 1989A) as being the reciprocal of the sum of the individual inverted cleft lengths $(L)$ :

$1 / \mathrm{TR}=1 / \mathrm{L} 1+1 / \mathrm{L} 2+\ldots+1 / \mathrm{Ln}$
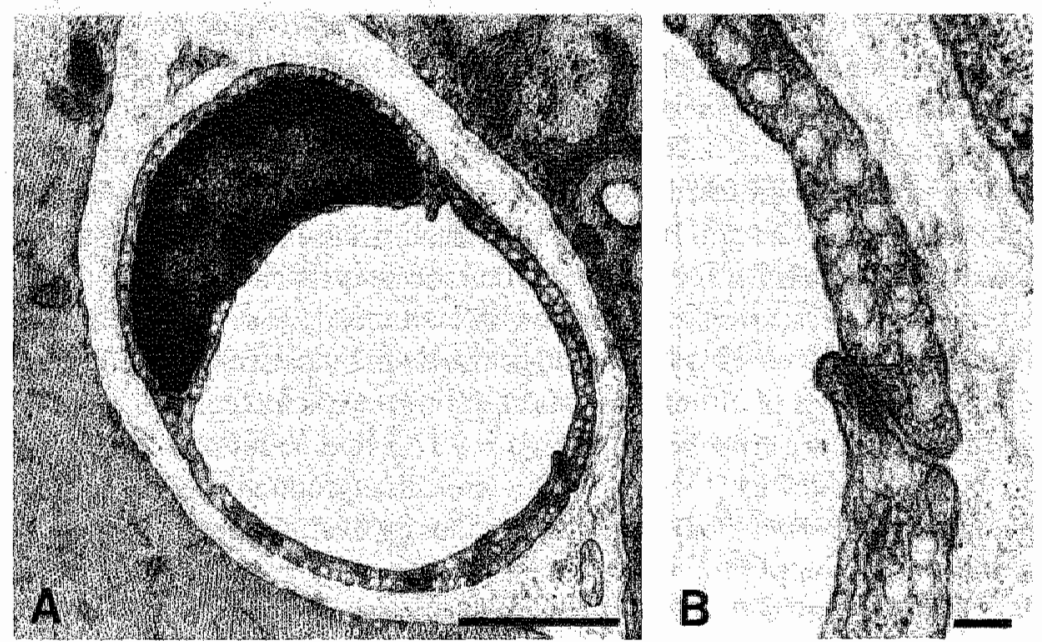

Fig. 4.1. Electronmicrograph of a capillary (A), and a capillary segment $(B)$ of soleus muscle fixed by perfusion with paraformaldehyde. Note the good preservation of ultrastructure. Bars, $1.0 \mu \mathrm{m}(\mathrm{A})$, and $0.1 \mu \mathrm{m}(B)$.

\section{Statistics}

The results are presented as means (of mean values per rat) \pm standard deviations. Differences were evaluated for statistical significance by means of a paired Student's $t$ test. Clustering of fiber types was tested by means of a Chi-square test.

Differences were considered to be statistically significant at p-values $<0.05$. 


\subsection{RESULTS}

Specificity of RIIDI0-antibody to type I muscle fibers.

With the staining procedure for myosin ATPase activity used, it was possible to identify three groups of fiber types in the muscle (type I, ILA/IIB and IIC), as can be seen in Figure 4.2C. Staining for myosin ATPase activity on cryosections failed when the muscles were fixed in $\mathrm{PF}, \mathrm{PF} / \mathrm{GA}$, or $\mathrm{GA}\left(4^{\circ} \mathrm{C}\right.$, overnight). Figure 4.2 shows consecutive cryosections of soleus muscle stained for myosin ATPase activity and labeled with R11DIO-antibody, respectively. As can be seen, the anti-cardiac myosin antibody RI1D10 cross-reacts with all type I fibers of soleus muscle, whereas no type II fibers are labeled. This is indicative of a good specificity of the antibody for type I fibers. It is essential that $\mathrm{GA}$ is omitted from the fixative because the
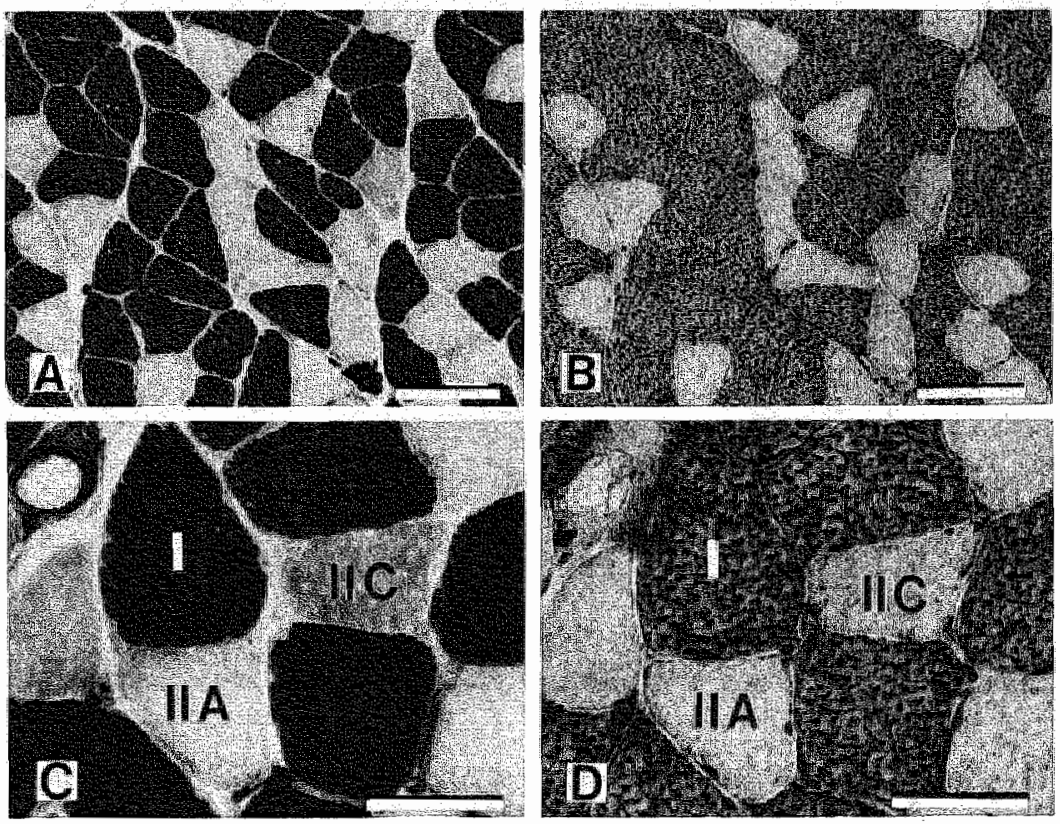

Fig. 4.2. Light microscopic cross-sections of frozen unfixed soleus muscle. $(A)$ and $(C)$ : sections stained for myosin $A T P$-ase activity after preincubation at $p H=4.3$. (B) and (D): immunostained by anticardiac monoclonal antybody R11D10. $(A)$ and $(C)$ are consecutive sections of $(B)$ and $(D)$, respectively. Only type I fibers are recognized by the antibody. I =type I fiber, IIA =type IIA fiber, IIC = type IIC fiber. Bars, $100 \mu m$ (A and B) and $50 \mu m(C$ and D). 
II fibers are labeled. This is indicative of a good specificity of the antibody for type $I$ fibers. It is essential that GA is omitted from the fixative because the addition of a small amount of GA $(0.5 \%)$ to the PF-fixative or replacement of the PF by GA (2.5\%) prevented immunohistochemical labeling of type I muscle fibers by RI1D10 on cryosections.

\section{Morphology of soleus muscle tissue.}

Type I and type II muscle fibers could be identified in Epon sections by comparing them with their paraffin embedded counterpart sections stained with RIID10antibodies (Figure 4.3). Morphology of the soleus muscle at the light as well as the electron microscopic level was well preserved. According to the classification of Bennett et al. (Bennett et al., 1959), type l-related as well as type II-related capillaries were of the A-1- $\alpha$-type, having a continuous basal membrane, an uninter-
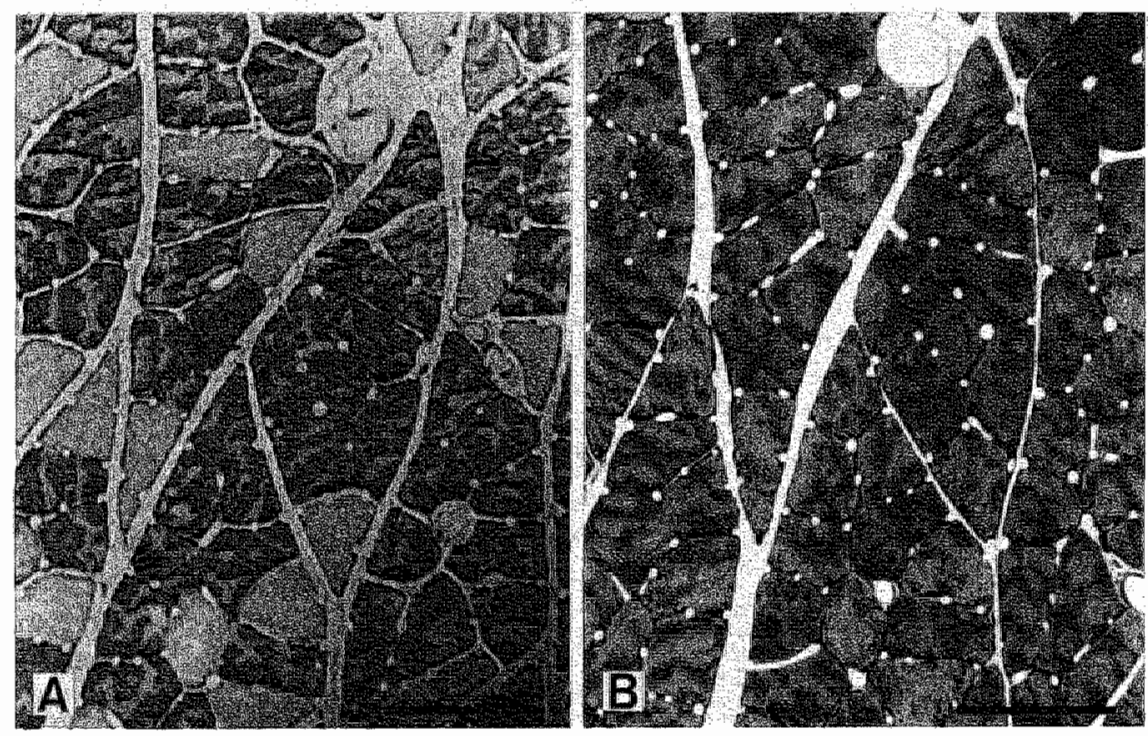

Fig. 4.3. Light microscopic cross-section of soleus muscle fixed by perfusion with paraformaldehyde. (A) paraffin embedded tissue immunostained for type I fibers by the monoclonal antybody RIID10. Type I fibers are stained dark, type II fibers are light. (B) Epon-embedded section of matched muscle tissue. Arrows indicate the same muscle fiber in both sections. Muscle fibers can be identified in the Eponembedded material. Bars, $100 \mu \mathrm{m}$. 
Distribution and morphometry of soleus muscle fibers.

The soleus muscle consisted predominantly of type I fibers $(77.8 \% \pm 6.3 \% ; 10$ rats; 200-350 fibers per rat). Type II muscle fibers were surrounded by $83.3 \pm 12.0 \%$ type I fibers ( 4 rats, 30 fibers per rat), which was significantly higher than expected on the basis of a random distribution of fiber types $(p<0.025)$.

The mean cross-sectional area of the type I and type II muscle fibers was $1349 \pm$ $123 \mu \mathrm{m}^{2}$ and $1295 \pm 121 \mu \mathrm{m}^{2}$, respectively $(\mathrm{p}>0.1)$. The number of capillaries around type I and type II muscle fibers was $5.67 \pm 0.61$ and $5.45 \pm 0.66$, respectively $(p>0.1)$. These data indicate that for type $I$ and type II fibers, the number of surrounding capillaries per unit cross-sectional muscle fiber area is comparable.

\section{Morphometry of soleus capillaries.}

Morphometric data of capillaries in relation to the number of surrounding muscle fibers and in relation to fiber type are presented in Table 4.1 and 4.2, respectively.

STEM video recordings of capillaries selected at random, showed that the number of capillaries situated between two fibers was about twice as high as that of capillaries situated between three or (very rarely) more fibers. Lumina of capillaries situated between two fibers were significantly more oval than capillaries situated between more than two fibers. The number of capillary lumina with extreme maxi$\mathrm{mal} / \mathrm{minimal}$ diameter ratios $>1.5$ was about twice as high for capillaries situated between two fibers (23.6\%) as compared with that of capillaries between more than two fibers $(12.8 \%)$. Lumina of all measured capillaries situated between two fibers

Table 4.1. Luminal paraneters of capillaries situated between two, or more then two fibers of soleus muscle.

$\begin{array}{lll}\text { Capillary Iuminal parameters } & \text { Capillaries } & \text { Capilluries } \\ & \text { between two } & \text { between three } \\ \text { fibers. } & \text { or nore fibers }\end{array}$

$\begin{array}{lcc}\text { cross-sectional area }\left(\mu \mathrm{m}^{2}\right) & 13.29 \pm 1.38 & 16.92 \pm 2.29 \\ \text { minimal diameter }(\mu \mathrm{m}) & 3.46 \pm 0.17 & 4.03 \pm 0.28 \\ \text { maximal/minimal diameter } & 1.42 \pm 0.14 & 1.26 \pm 0.06\end{array}$

All values indicate means ( \pm standard deviation) of 7 rats (72-98 capillaries per rat). Morphometric data were collected from STEM video images. $P_{\text {-value }}$ (paired Student's t test) $<0.02$ for all parameters. 
had a significantly smaller cross-sectional area (about $21 \%$ ), and minimal diameter (about 14\%) than lumina of capillaries situated between more than two fibers (Table 4.1). These results show that for comparison of capillary lumina in relation to fiber type, the position of the capillaries involved has to be taken into account.

The morphometric results of a total of 35 type II related capillaries and 40 type I related capillaries of 7 rats are presented in Tabel 4.2. These data do not include capillaries with minimal/maximal luminal diameter ratios $>1.5$. Of the measured parameters associated with the capillary lumen or endothelial cells, non was different for tIr-as compared with tIIr-capillaries ( $p \geq 0.1$ ).

Table 4.2. Parameters of capillaries related to type I (tIr) and type II (tIIr) soleus muscle fibers of rat.

\begin{tabular}{|c|c|c|}
\hline Parameters & $\begin{array}{l}\text { tfr" } \\
\text { capillaries }\end{array}$ & $\begin{array}{l}\text { tllr- } \\
\text { capillarics }\end{array}$ \\
\hline \multicolumn{3}{|l|}{ Capillary Lumben } \\
\hline cross-sectionall areng $\left(\mu \mathrm{n}^{2}\right)$ & $9.59 \pm 1.39$ & $11.70 \pm 3.67$ \\
\hline minimal diameter $(\mu \mathrm{m} n)$ & $3.10 \pm 0.211$ & 3.3410 .64 \\
\hline maximal/minimal diameter & $1.26 \pm 0.06$ & $1.22 \pm 0.05$ \\
\hline perimeier $(\mu \mathrm{m})$ & $11.5 \pm 0.9$ & $12.5 \pm 2.0$ \\
\hline \multicolumn{3}{|l|}{ Endothelial cytoplasm } \\
\hline cross-sectional are $\left(\mu \mathrm{m}^{2}\right.$ ) & $2.30 \pm 0.24$ & $2.36 \pm 0.24$ \\
\hline mean thickness $(\mu \mathrm{m})$ & $0.115 \pm 0.01$ & $0.15 \pm 0.02$ \\
\hline \multicolumn{3}{|l|}{ Mitochondria } \\
\hline \% of cytoplasm & $3.60 \pm 1.89$ & $2.55 \pm 1.29$ \\
\hline \multicolumn{3}{|l|}{ Vesicles } \\
\hline $\begin{array}{l}\text { number/ } / \mathrm{m}^{2} \text { cytoplasm } \\
\text { luminal vesicles: }\end{array}$ & $46.5 \pm 8.3$ & $42.7 \pm 11.0$ \\
\hline $\begin{array}{l}\text { luminal vesicles: } \\
\text { number/ } \mu \mathrm{m} \text { luminal membrane }\end{array}$ & $1.01 \pm 0.38$ & $123+0.41$ \\
\hline $\begin{array}{l}\text { number / /m lumunal membrame } \\
\text { membrune length. }\end{array}$ & $0.20 \pm 0.01$ & $0.19 \pm 0.01$ \\
\hline \multicolumn{3}{|l|}{ cyloplatimic vesicles: } \\
\hline number/ $/ \mu m^{2}$ cyloplasm & $23.5 \pm 5.3$ & $20.0 \pm 4.9$ \\
\hline cross-sectional ares $\left(\mathrm{nm}^{2}\right)$ & $3,140 \pm 3.18$ & $2,970 \pm 124$ \\
\hline \multicolumn{3}{|l|}{$\begin{array}{l}\text { abliminal vesicles: } \\
\text { numberdum abluminal }\end{array}$} \\
\hline membrane. & $2.46 \pm 0.61$ & $2.37 \pm 0.50$ \\
\hline membrane length & $0.19 \pm 0.01$ & $0.19 \pm 0.02$ \\
\hline \multicolumn{3}{|l|}{ Interendothdial cledts } \\
\hline number/catpillary & $10.80 \pm 0.24$ & $1.80 \pm 0.37$ \\
\hline transport resistance (TR) & $0.24 \pm 0.07$ & $0.25 \pm 0.17$ \\
\hline \multicolumn{3}{|l|}{ Banal membrane } \\
\hline mean thickness (nm) & $47 \pm 6$ & $47 \pm 10$ \\
\hline
\end{tabular}

All values indicate means ( \pm standard deviation) of 7 rats (4-7 capillaries per rat). Capillaries with a ratio of maximal/minimal luminal diameter $>1.5$ are not included into the analysis. Morphometric data were collected from CTEM micrographs. P-value (paired Student's t test) $\leq 0.10$ for all parameters. 


\subsection{DISCUSSION}

To investigate the (ultra)structure of capillaries supplying slow twitch (type I) and fast twitch (type II) fibers, a retrieval method which combines muscle fiber typing with (ultra)structural preservation of the tissue cells was used. Following perfusion fixation, fibers in sections of plastic embedded rat soleus muscle could be retrieved in their immunostained paraffin embedded counterparts and subsequently classified. Using this method, morphometry of capillaries in relation to the type of fibers they supply, was studied in plastic embedded material. A high simillarity existed between type I and type II muscle fibers concerning cross-sectional area, capillarization and morphology of their related capillaries.

A direct combination of muscle fiber typing with preservation of muscle (ultra)structure is difficult. Histochemical muscle fiber typing requires the use of unfixed cryosections (Dodson et at., 1987), which results in collapsed capillaries and generally poor ultrastructural preservation. On the other hand, conventional fixation and embedding methods for (electron)microscopy do completely inhibit histochemical reactions. The recent development of fiber type specific antibodies that can be used on chemically fixed tissue (Dodson et al., 1987, Havenith et al., 1989), made it possible to combine fiber typing with the preservation of tissue (ultra)structure. In this study, good results were obtained by perfusing rat soleus muscles with a 4\% paraformaldehyde fixative. For fiber typing an anticardiac myosin monoclonal antibody (RIIDIO), that reacts with type I (slow twitch), but not with type II (fast twitch) muscle fibers, was used. Tissue morphology of the Epon-embedded material, appropriate for morphometric analysis, was well preserved. Because direct labeling of Epon-embedded sections failed, an indirect retrieval method was developed. By comparing the muscle fibers embedded in Epon with their counterparts on the immunostained sections, their type could unequivocally be determined.

Antibodies suitable for muscle fiber typing on fixed, paraffin embedded material allow long preservation of the tissue. Its use lowers the risks of infection of the investigator by unfixed pathogens. The cross-reactions of antibodies directed against cardiac myosin and type I muscle fiber myosin (Sartore, 1981; Havenith et al., 1989) is compatible with the biochemical similarities between myosin of cardiac and type I muscle cells (Whalen et al., 1979). Because soleus muscle of rat (Brooke and Kaiser, 1970; Pullen, 1977; Luginbuhl et al., 1984) does not contain type IIB fibers and type IIC fibers in soleus muscle are rare (Brooke and Kaiser, 1970; Gray and Renkin, 1978; Myrhage, 1978; Sullivan and Pittman, 1984), in the present study we probably discriminated mainly between type I and type IIA fibers.

The morphometric analysis of muscle fibers and capillaries was performed on video recordings or photographs of semithin and ultrathin sections. The cross-sectional muscle fiber area (about $1,300 \mu \mathrm{m}^{2}$ ), and fiber capillarization, i.e., the number of capillaries around one fiber, was about equal for both type I and type II soleus 
muscle fibers (approximately 5.6 per fiber). This indicates that the number of capillaries per unit area of the adjacent fiber was the same for both fiber types, suggesting that both types were equally supplied by capilaries. The cross-sectional fiber areas found correspond well with the mean values found in rat soleus as reported in a previous study (Peeze Binkhorst et al., 1989A). However, Pullen (1977) found in cryofixed soleus muscles of Wistar rats of about the same age, that the cross-sectional area of type I fibers was about $50 \%$ larger than that of type II fibers. These contradicting results may be due to strain differences or to differences in treatment of the tissue. Type I fibers are in general more aerobic-oxidative, and type II fibers are more glycolytic in nature, altbough small discrepancies might exist (Peter et al., 1972; Pullen, 1977; Nemethet al., 1979). Because aerobic-oxidative fibers, when active, depend more on oxygen supply, and thus on an effective (micro)circulation, than glycolytic fibers, differences in density and/or structure of capillaries related to different fiber types are feasible. Howewer, no such differences were found.

Capillaries situated between two fibers had a significantly smaller cross-sectional Iuminal area (about 21\%) than capillaries situated between three or more fibers. The difference between the capillary lumina of both groups was even larger when the minimal luminal diameters were compared, probably because this parameter is virtually not influenced by the angle at which the capillaries were cut. The findings indicate that the wider capillaries are situated at the corners of the muscle fibers where, in general, most space appears to be available. The larger ovality of lumina of capillaries situated between two fibers is probably the result of a higher incidence of capillaries running obliquely to the muscle fiber orientation, e.g. interconnecting capillaries, in this group.

Because in cross-sections of the soleus muscle about $80 \%$ of the fibers were of type I and only $20 \%$ of type II, capillaries situated between three or more type II fibers were rare as compared with capillaries situated between three or more type I fibers. A random selection of fiber type related capillaries would therefore yield groups with capillaries not only situated between different fiber types, but also surrounded by different numbers of fibers. Therefore, when capillaries were studied in relation to fiber type, capillaries situated between more than two fibers, were excluded. Of all measured capilary morphometric parameters, the values were similar for tIr- and tIIr-capillaries. The general morphological similarity between tIrand tIr-capillaries is probably not due to a dominant determination of capillary morphology by the abundant type I fibers, because the endothelial cells of tIIr-capillaries were never adjacent to type I fibers at the location of sectioning.

The results of the present study indicate that, as far as rat soleus is concerned, both fiber types I and II are equally supplied with blood because:

1. The number of capillaries per unit fiber area was comparable for tilr and thr capillaries.

2. TIr and tIIr capillaries were very similar in nearly all morphometric aspects.

3. Arterioles were rare in muscle cross-sections as compared with capillaries. This indicates that arterioles supply many capillaries in the soleus muscle. Therefore, the 
non-clustered distribution of type II fibers indicates that most arterioles supply capillaries of both fiber types.

Although no physiological parameters were measured, no morphological basis for fiber type related differences in capillary flow and/or exchange of substances were found in soleus muscle of rat. Therefore, the finding that endurance exercise causes focal degeneration of soleus type I fibers only (Kuipers et al., 1983), cannot be explained by a less effective organization and/or (ultra)structure of tIr capillaries as compared with tIIr capillaries. In addition, it is unlikely that the relatively large luminal area of capillaries around degenerated fibers (Peeze Binkhorst et al., $1989 \mathrm{~A}$ ), can be attributed to a selection of capillaries adjacent to type I fibers. Therefore, the capillaries adjacent to these affected fibers were probably dilated as a result of diffusion of yet unidentified local factors related to the exercise induced degeneration.

In this study we combined muscle fiber typing with the preservation of muscle and capillary (ultra)structure in the soleus muscle of rat. Capillaries between two muscle fibers were significantly smaller than capillaries between site of contact between three or more fibers. A good similarity between size and capillarization of slow and fast twitch fibers, and the morphometry of fiber type related capillaries were found.

\subsection{SUMMARY}

Capillary (ultra)structure in relation to its position between muscle fibers and in relation to the type of the surrounding muscle fibers (slow twitch (type I) vs. fast twitch (type II)), was investigated in the soleus muscle of the rat.

Preservation of muscle (ultra)structure and fiber typing were combined using a retrieval method. Soleus muscles of rat were fixed by perfusion with paraformaldehyde fixative. Parts of the muscle were cut in two halves. One half was embedded in paraffin for fiber typing, the counterpart was embedded in plastic (Epon) for morphometric analysis. Fiber typing was performed immunohistochemically using an anti-cardiac myosin antibody that selectively reacted with skeletal muscle type I fibers. Type I and type II fibers in sections used for morphometry were classified by comparing them with their immunostained counterpart.

The cross-sectional luminal area of capillaries situated between two muscle fibers was about $21 \%$ smaller than that of capillaries situated between more than two muscle fibers. The cross-sectional area and capillarization of type I and type II fibers were undiscernable. Capillaries situated exclusively between type I or exclusively between type II fibers were similar as far as their morphology and morphometry are concerned. In addition, the results indicate that in general arterioles supply capillaries of both muscle fiber types. 
The morphological data obtained indicate that, at least in the soleus muscle, blood supply and/or exchange of material between blood and muscle fibers is similar for type I and type II fibers. 


\subsection{INTRODUCTION}

Endurance running can induce focal muscle fiber degeneration in humans (Geller, 1973; Warhol et al., 1985; Hoppeler, 1986) and in animals (Lindholmet al., 1974; Armstrong et al., 1983; Kuipers et al., 1983; Peeze Binkhorst et al., 1989A). In animals this degeneration is associated with infiltration of inflammatory cells. The sequence of events of exercise-induced morphological changes has a striking resemblance to that due to ischemic necrosis (Sanderson et al., 1975; Mäkitie and Teräväinen, 1977). However, it is unknown whether ischemia contributes to exercise induced fiber degeneration. In rat soleus muscle, (electron)microscopic observations did not reveal obstructions of blood vessels preceeding the exercise induced fiber degeneration observed at 24 hours after rumning. However, at 6 hours after running the muscle fiber degeneration was preceeded by swelling of the muscle fibers of $28 \%$ as compared to control (Peeze Binkhorst et al., 1989A). This swelling could have lead to an increase in intramuscular tissue pressure, causing muscle fiber necrosis, especially since long lasting increases in intramuscular pressure result in muscle fiber necrosis (Reneman, 1968 and 1975; Matsen, 1975; Fridén et al., 1988).

In the present study the time course and magnitude of post-exercise swelling was studied in rat soleus muscle. Besides, it was investigated whether muscle swelling leads to an increase in intramuscular pressure. The experiments were performed on non-exercised control rats and exercised rats up to one day after running. Intramuscular pressure was measured with glass micropipettes and a servo-nulling counterpressure system (Wiederhielm et al., 1964). 


\subsection{MATERLAL AND METHODS}

\section{Experimental and exercise procedures}

Untrained, male Brown Norwegian rats $(n=27), 11-30$ weeks old and weighing $210-370 \mathrm{~g}$, were used. The animals were housed in a temperature controlled environment $\left(23-26^{\circ} \mathrm{C}\right)$ and fed with rat chow (type 1210 , Hope Farms) and water ad libitum.

The rats were randomly assigned to either a non-exercised control group or an exercised group. The rats of the exercise group were placed on a rodent treadmill (Quinton) with a positive slope of $10^{\circ}$ and were stimulated to run by an intermittent electrical current (duration 0.1 s) that was applied to a resting grid each five seconds. Mean effective running time was approximately $90 \%$ during the initial adiaptation period ( 15 minutes at $875 \mathrm{~m} / \mathrm{h}$ ) and approximately $65 \%$ during the subsequent exercise period $(60$ minutes at $1620 \mathrm{~m} / \mathrm{h})$. During running room temperature was kept at $23-26^{\circ} \mathrm{C}$.

The animals of the non-exercised control group and those of the exercised group were anesthetized with sodium-pentobarbital (60\% solution ( $N$ arcovet); $1 \mathrm{ml} / \mathrm{kg}$ body weight, i.p.). To assess fiber necrosis or tissue swelling, both soleus muscles were dissected in a subgroup of non-exercised animals $(n=6)$ and in subgroups of exercised animals at $3,6,9$ or 24 hours after running $(n=6$ for each group). In an other group of animals tissue pressure was measured in the soleus muscles in vivo up to one day after running $(n=11)$ and in control animals $(n=5)$.

\section{Muscle cryofixation}

Muscle wet weight and swelling of muscle fibers was determined from cryofixed muscles because cryofixation produces virtually no swelling or shrinkage of the tissue, in contrast to most other chemical fixation and embedding methods (Glauert, 1975; Aherne and Dunnill, 1982). Rats of similar weight (210-290 g) were used in this part of the study, because muscle weight is correlated with body weight.

Swelling of the muscle fibers and post-exercise fiber degeneration, if any, was determined from cross-sections of muscles, cryofixed at a standard length. Rats were put in a lateral position. The lateral side of the soleus muscle of the right hind limb was exposed by incision of the overlying skin and muscle tissue. Small marks were tattooed on each of the distal ends of the soleus, using a small injection needle (outer diameter $0.5 \mathrm{~mm}$ ) filled with India ink. The hind limb was then fixed in a standard position (hip $135^{\circ}$, ankle $90^{\circ}$, knee stretched) and the distance between the tattoos was measured. The tendons were cut. The muscle was dissected and gently stretched using a self-retaining retractor ( $7 \mathrm{~cm}$, blunt spongs, Medicon Instruments) until the 
distance between the tattoos had reached its initial value. In this position, the muscles were quickly frozen in melting isopentane. To determine muscle wet weight, the soleus muscle of the contrallateral hind limb was dissected and quickly washed in saline. The muscle was then frozen in liquid nitrogen to prevent dehydration. Care was taken to remove excess fluid on the outside of the muscle. All cryofixed muscles were stored at $-60^{\circ} \mathrm{C}$ up to one week, before further processing.

Muscle morphology and morphometry

Cryosections (10 $\mu \mathrm{m}$ thick) of the midportion of the muscle, fixed at its standard length, were cut perpendicular to the fiber orientation on a cryostat at $-20^{\circ} \mathrm{C}$. The sections were mounted on glass slides, air dried, stained with haematoxylin and erythrosine (10 min and $1 \mathrm{~min}$ at room temperature, respectively), and embedded in Entellan. The tissue sections were checked for degenerated muscle fibers and morphometrically analyzed using light microscopy (objective $25 \mathrm{x}$, numerical aperture 0.45 ).

Video images of the tissue were used for morphometric analysis, employing a Kontron Mop-Videoplan digitizer system (Zeiss, FRG). Optical magnification was 16x. For each rat the mean fiber cross-sectional area (FA) was determined from fibers present in a rectangular window of six video frames (total area $0.44 \mathrm{~mm}^{2}$ ) muscle tissue without large blood or lymph vessels. Fibers overlying the top and/or right hand side of a window were included in the analysis. Fibers overlying the bottom and/or left hand side of a window were excluded from analysis. Each window contained 21-38 muscle fibers. The relative fiber area (RFA) was calculated as:

$$
\text { rellative fiber area }=\frac{\text { total fiber area }\left(\mathrm{mm}^{2}\right)}{0.44}
$$

If fibers and non-fiber tissue swell to the same extent, then the the relative fiber area remains constant. Because the muscles analyzed were fixed at a standard length, changes in the fiber and non-fiber area are similar to changes in their volume. The change in muscle volume after exercise, as compared to control, was callculated on the basis of the measured cross-sectional fiber area using the relation:

$\frac{M V_{\text {exercise }}}{M V_{\text {control }}}=\frac{\text { FAexercise } / \mathrm{RFA}_{\text {excrcise }}}{\mathrm{FA}_{\text {control }} / \mathrm{RFA}_{\text {control }}}$

in which $\mathrm{MV}=$ muscle volume. Because fiber cross-sectional area depends on body mass (see results), FA was expressed relative to body mass.

The change in the muscle non-fiber area can be calculated using the relation: 


$\frac{\text { NFAexercise }}{\text { NFAcontrol }}=\frac{\text { FAexercise }}{\text { FAcontrol }} * \frac{\text { RNFAexercise }}{\text { RNFAcontrol }}$

in which RNFA = relative non-fiber area $=1-\mathrm{RFA}$.

\section{Muscle wet weight}

Muscle wet weights were determined on a microbalance (Satorius H51, Zeiss, FRG). Because muscle wet weight depends on body mass (see results), muscle wet weight was expressed relative to body mass. Because changes in muscle wet weight are similar to changes in muscle volume, we were able to compare the changes in muscle volume with the changes in muscle volume calculated with formula (2).

\section{Intramuscular pressure measurements}

Intramuscular pressure was measured using a servo-nulling counterpressure system (model 4A; IPM; USA) a technique originally described by Wiederhielm et al. (1964). In short, a micropipette (inner tip diameter $<1 \mu \mathrm{m}$ ) is inserted into the tissue. The instantaneous electrical resistance of the pipette is determined by the position of the interface between the relatively high conductant $2 \mathrm{M} \mathrm{NaCl}$ solution in the pipette and the relatively low conductant tissue fluid. The pipette is connected to a pressure system. By comparing the resistance of the micropipette (3-7 Mn) with a preset value, the pressure difference between the muscle interstitial fluid and the servo system can be kept constant. The counterpressure generated by the servo system was recorded with a pressure transducer (Central Technology Company, U.S.A.) connected to a multichannel playsiological recorder (Picker/Schwartzer, FRG). A sensitivity of $5 \mathrm{~mm}$ deflection/ $\mathrm{mm} \mathrm{Hg}$ was used. The accuracy of the pressure measurements was better then $0.1 \mathrm{~mm} \mathrm{Hg}$. The pressure transducer was calibrated before each measurement using a water column. Zero pressure level was recorded before and after each measurement in the thin fluid layer covering the muscle.

Recordings were accepted when (1) changing the reference resistance of the measuring system resulted in an immediate adjustment of the $2 \mathrm{M} \mathrm{NaCl} /$ interstitial fluid interface, as indicated by a returning of the recorded pressure to its original value. We generally also tested whether a slight increase of the feedback gain resulted in relatively large rapid variations around the original value, demonstrating that the tip was patent, (2) pressures were constant during the short recording periods (typically $5 \mathrm{~min}$ ). An ongoing increase of the recorded pressure indicated an increasing obstruction of the pipette tip. In these cases, the recordings were excluded, (3) pressures returned to stable zero-values immediatly after the pipette had been 
withdrawn into the thin fluid layer covering the muscle, indicating that the tip of the pipette was still patent.

Using these criteria, the success rate was low. It was difficult to find free fluid pockets and, if found, the position of the tip of the pipette was critical and the pressure recordings highly sensitive to movement.

\section{Micropuncture experiments}

After anesthesia, 16 rats were either intubated directly or a tracheotomy was performed to facilitate breathing of the animal. The left femoral artery was canmulated to measure arterial pressure (pressure transducer and recorder as described above). Only when intramuscular pressures had to be measured immediately after ruming, the femoral artery was not cannulated. Additional anesthesia was given intraperitoneally whenever necessary. The rats were placed with their left lateral side on a heating pad $\left(37^{\circ} \mathrm{C}\right)$. To minimize movement, the foot of the right hind limb was fixed without trauma between foampads with the hind limb joints positioned in the same way as discribed for the cryofixation procedure. A small needle was inserted under the skin covering the right hip and served as electrical ground. Subsequently, an incision of about $3 \mathrm{~mm}$ was made in the skin overlaying the lateral side of the soleus. Muscle tissue overlaying the soleus was split carefully untill part of the lateral side of the soleus became accessible.

A micropipette was mounted on a micromanipulator. Using a stereomicroscope (Zeiss; magnification $25 x$ ) the soleus muscle was punctured at an angle of approximately $45^{\circ}$ to the horizonal plane. If the tip of a micropipette was too flexible to penetrate the fascia, a small incision $(<0.5 \mathrm{~mm}$ ) was made in the fascia, using the tip of an thin injection needle. To prevent dehydration, the muscle was subsequently covered with a thin film of paraffin oil. For pressure measurements the pipettes had to be slightly withdrawn after puncturing, until an appropriate free space was found. Measurements were performed at different sites of the muscle at depths ranging from about 0.5 to $2 \mathrm{~mm}$, and without visible distortion of the muscle.

Intramuscular pressure measurements were performed in exercised rats between 18 min and $23 \mathrm{~h} 19 \mathrm{~min}$ after running, and in control rats. Duration of anesthesia at the moment of puncturing varied from $18 \mathrm{~min}$ up $106 \mathrm{~h} 15 \mathrm{~min}$. Intramuscular pressures were recorded during 5 minutes, and, if the tip of the pipette was not moved, pressure recordings remained constant for at least 30 minutes.

\section{Accuracy of pressure measurements in muscle}

It could be argued that puncturing of the fascia loweres intramuscular pressure. Therefore, we tested our procedure using a confined compression system (see Figure 5.1). A soleus muscle was freshly dissected and the distal ends cut off. Then the muscle was placed in a rigid tube just wide enough to contain it (i.d. $4 \mathrm{~mm}$ ). Sub. 


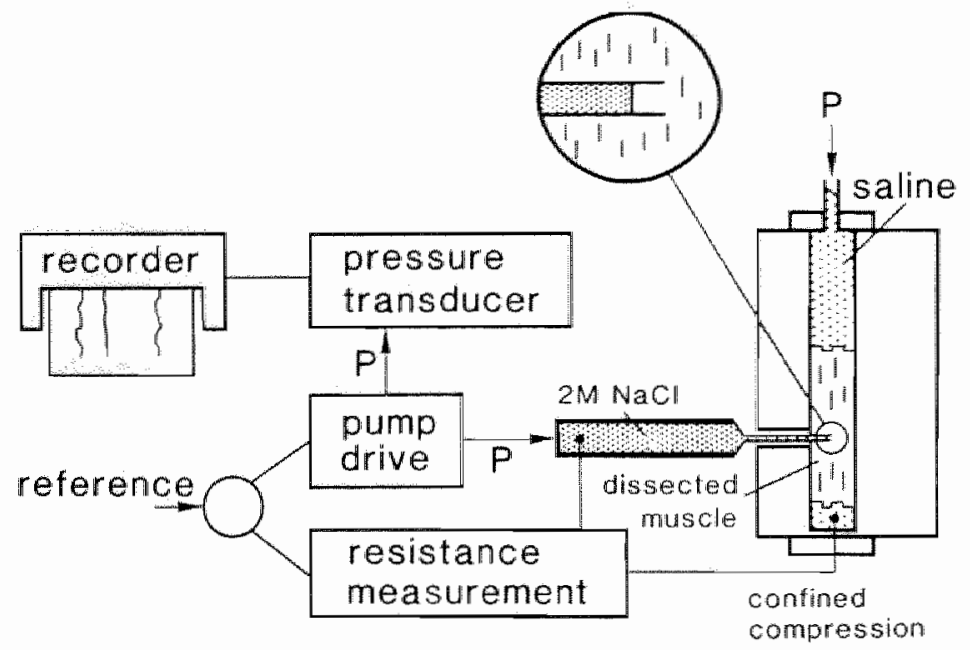

Fig. 5.1. Setup for evaluation of the micro pipette technique to measure increases in intramuscular tissue pressure. The confined compression system contains a freshly dissected rat soleus muscle. Pressure is imposed on the muscle via a mercury pressure column. Intramuscular pressure is measured with the seno-nulling counterpressure system via a micropipette that is inserted into the muscle via a small hole (diameter $0.7 \mathrm{~mm}$ ). The enlarged insert shows the position of the interface between the tissue fluid and the $2 \mathrm{MNaCl}$ solution in the pipette.

sequently, the tube was filled with saline at both ends and subsequently sealed. The system was electrically grounded. Pressure was applied to the muscle using a mercury column connected to the saline. After pressurizing the system, the glass pipette was inserted into the midportion of the muscle via a lateral hole (diameter $0.7 \mathrm{~mm}$ ) in the tube. This hole was small enough to prevent the muscle from herniating out of the system, even at the highest pressures imposed. Pressures imposed on the muscle (up to $150 \mathrm{~mm} \mathrm{Hg}$ ) were well recorded by the servo-nulling counterpressure system, as is illustrated in Figure 5.2. This indicates that puncturing of the fascia did not reduce intramuscular pressure.

\section{Statistics}

Data are presented as individual values, as mean \pm S.D. (standard dewiation), or as the mathematical function of a linear regression line with corresponding coefficient of correlation ( $r$ )-value, whenever appropriate. 


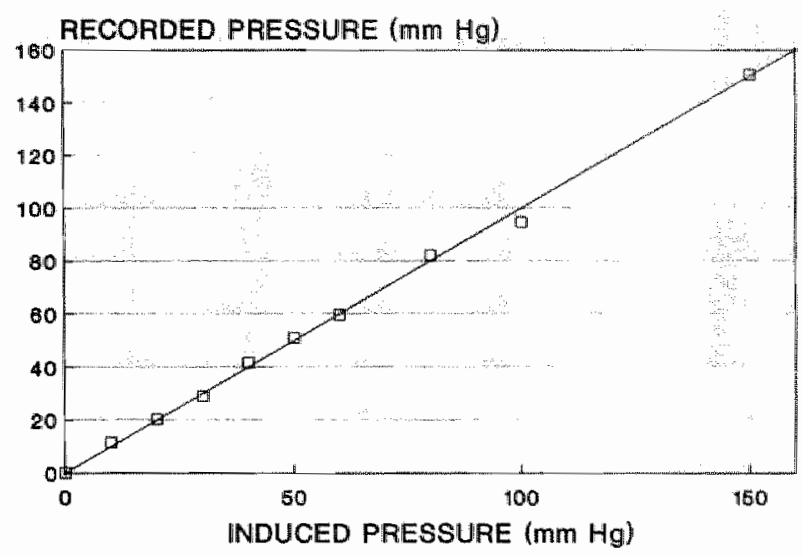

Fig. 5.2. Tissue pressure data of rat soleus muscle, using the confined compression system. Intramuscular pressure changes to the same extent as the pressure exerted on the muscle by the mercury pressure column up to at least $150 \mathrm{~mm} \mathrm{Hg}$. Line of identity is indicated.

For statistical anialysis a one-sided Student's $t$ test was used to compare mean values, a $\mathbf{F}$ test was used to compare variance ratios and the calculated coefficient of correlation was used to calculate the a possible correlation between two parameters. Differences between groups were considered to be statistically significant at $\mathrm{p}<0.05$.

\subsection{RESULTS}

\section{Femoral artery pressure}

The mean femoral artery pressure immediately after catheterization varied between 75 and $120 \mathrm{~mm} \mathrm{Hg}$ (mean $95 \mathrm{~mm} \mathrm{Hg} ; \mathrm{n}=8$ ). Arterial pressures did not differ between exercised and non-exercised (control) rats.

\section{Muscle swelling}

The mean soleus muscle wet weight of control rats was $123 \pm 18 \mathrm{mg}$ and the mean cross-sectional muscle fiber area $2,014 \pm 155 \mathrm{\mu m}^{2}(\mathrm{n}=6$ rats $)$. The area of muscle fibers was $90.5 \pm 2.3 \%$ of the total cross-sectional area with exclusion of large blood and lymph vessels. Soleus muscle wet weight as well as cross-sectional fiber area 


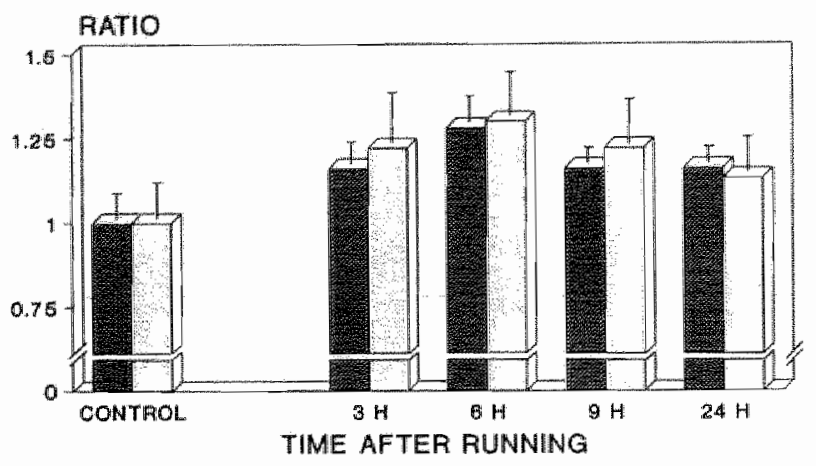

MUSCle WeT WeIaht DMUSCle Wolume A

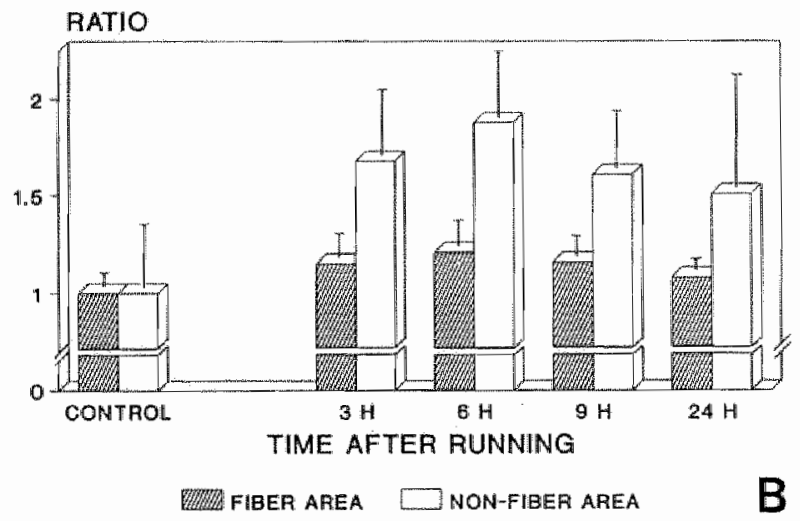

Fig. 5.3. Muscle wet weight and calculated muscle volume relative to body weight (A). Muscle fiber cross-sectional area and muscle non-fiber area relative to body weight (B). Each value relative to control values (set at 1.0) and as a function of time after running. Regions with large blood or lymph vessels were not included in the analysis of cross-sectional areas. Note the differences in vertical scales. All values obtained at $3,6,9$, and $24 \mathrm{~h}$ after running are significantly larger than control values (non-exercised rats; $P<0.05$ ). The increase in muscle wet weight and muscle volume did not differ $(P>0.10)$, but the increase of the fiber area was atways less than that of the non-fiber area $(P<0.05)$.

showed a significant correlation with rat body mass in each group (all values normalized to mean values per group; $\mathrm{p}<0.005 ; \mathrm{n}=30$; $\mathrm{r}$-values 0.69 and 0.55 , respectively). When divided by body mass, these values were independent of rat weight $(p>0.10 ; n=30 ; r$-values 0.14 and 0.13 , respectively). To be able to compare data, muscle wet weight and relative fiber cross-sectional area were expressed relative to body weight. The changes in soleus muscle wet weight and calculated muscle volume, cross-sectional fiber area and non-fiber area relative to the control values at 
various hours after running are presented in Figure 5.3. Muscle wet weight was increased at $3,6,9$ and 24 hours after running, being maximally ( $28 \%$ ) at 6 hours. The increase in muscle wet weight was associated with a similar increase in muscle volume, as calculated from muscle cross-sections. The increase in muscle swelling did not differ significantly from the increase in muscle wet weight, indicating that both methods were consistent. Swelling of the muscle fibers accounted for most of the increase in muscle volume, but swelling of the non-fiber area was several times larger than that of the fibers (see Figure 5.3).

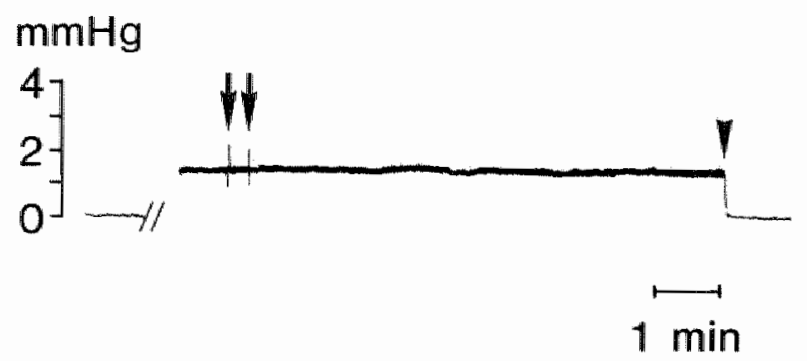

Fig. 5.4. Example of a tissue pressure recording from rat soleus muscle. Control situation. Arrows indicate the moment of changing the reference resistance of the measuring system. The measured pressure immediately retumed to its intial value. Arrowhead indicaics the moment of withdrawel of the pipette from the tissue into the thin fluid film covering the muscle (return to constant zero pressure).

\section{Intramuscular pressure}

An intramuscular pressure reconding in a control rat is shown in Figure 5.4. Intramuscular pressure values of a control rat, as measured at different durations of anesthesia, are presented in Figure 5.5. The variance of the intramuscular pressures measured in the control rats rats was larger within individual rats that between rats ( $p<0.005 ; 41$ measurements; $F$ test). These measurements show that in both exercised and control rats the intramuscular pressure measured did not change during periods of anesthesia of up to $6 \mathrm{~h} 50 \mathrm{~min}(\mathrm{r}=0.10$ for all control data combined; 5 rats, 43 measurements; $\mathrm{p}>0.10$ ).

Intramuscular pressures measured in exercised rats at different intervals after running are presented in Figure 5.6. Mean intramuscular pressure of control rats was $1.0 \pm 0.2 \mathrm{~mm} \mathrm{Hg}$ ( 5 rats; 43 measurements). Intramuscular pressure between $18 \mathrm{~min}$ and $23 \mathrm{~h} 19 \mathrm{~min}$ after running ( $1.3 \pm 0.3 \mathrm{~mm} \mathrm{Hg} ; 11$ rats; 116 measurements) did not differ significantly from control values. Even around the period of maximal muscle swelling (4-8 hours after running) no increase in intramuscular pressure was found, the mean value during this period being $0.9 \pm 0.3 \mathrm{~mm} \mathrm{Hg}$ ( $4 \mathrm{rats} ; 20$ measure- 
ments). In none of the animals any considerable change of intramuscular pressure during a certain period of time up to one day after running could be detected.

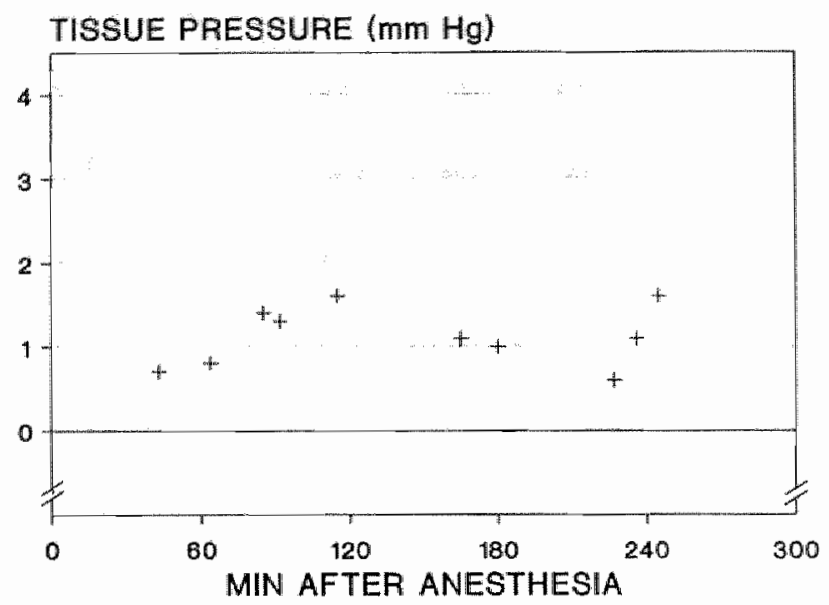

Fig. 5.5. Tissue pressure values in soleus muscle of a non-exercised control rat at different durations of anesthesia.

Muscle morphology

Degenerated muscle fibers, defined as muscle fibers invaded by inflammatory cells, were rare in control animals and in exercised animals during the first 6 hours after running. At 9 hours after running their number was increased and maximal numbers were found at 24 hours after running, confirming earlier findings by Kuipers et al. (1983). The number of invaded fibers found in one cross-section was variable and varied between 0.2 and 1.5 per $\mathrm{mm}^{2}$ at 24 hours after running.

\subsection{DISCUSSION}

The findings in the present study show that uphill running induces swelling of rat soleus muscle that reaches a maximum after 6 hours and is still noticeable after 24 hours. In addition, exercise induced focal muscle fiber necrosis that was most pronounced 24 hours after running, being the end of the observation period. The swelling, which preceeds fiber necrosis in time, was not associated with increased intramuscular pressure.

Swelling was observed in muscle fibers as well as non-fiber tissue, which resulted in an increase in muscle wet weight. Although non-fiber tissue swelled several times 
TISSUE PRESSURE $(\mathrm{mm} \mathrm{Hg})$

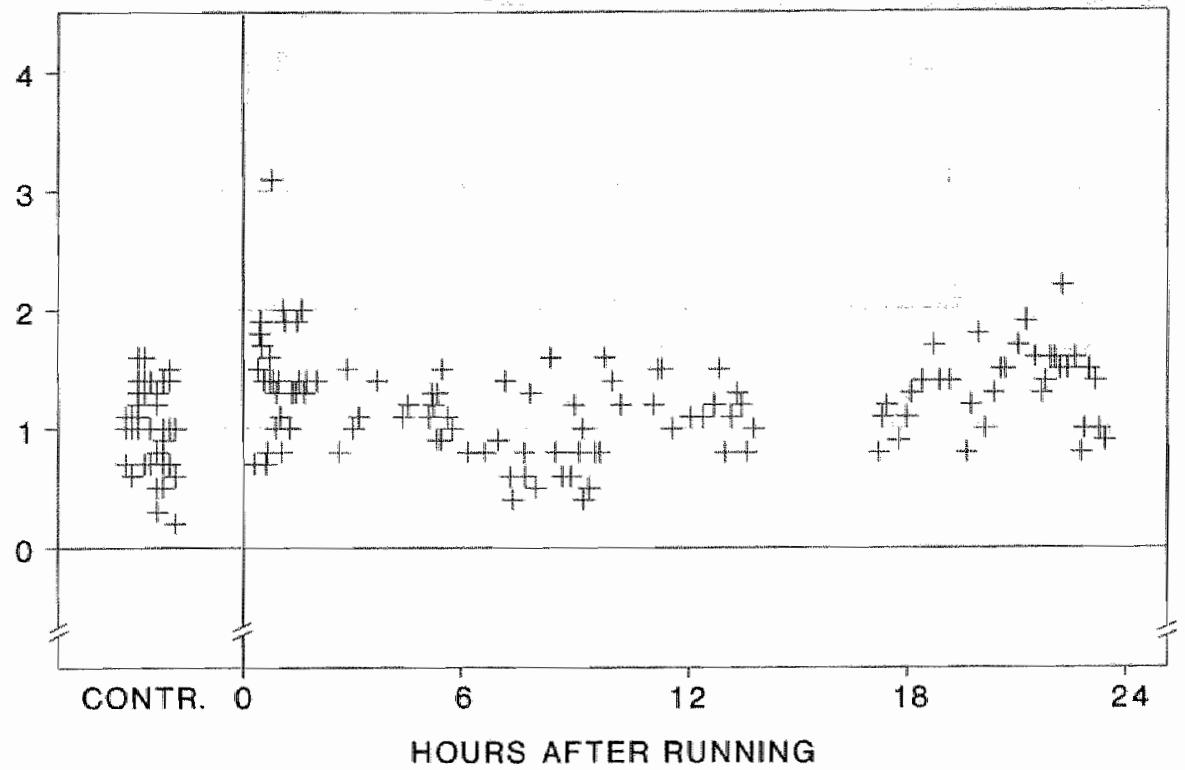

Fig. 5.6. Scatter plot of tissue pressures of rat soleus muscle before (control) and at different intervals after running. Combined data of 16 rats.

more than the muscle fibers, muscle swelling was dominated by swelling of fibers which comprise $83-91 \%$ of the muscle volume. Swelling of non-fiber tissue likely results from swelling of the interstitium because capillaries do not change their diameter under these circumstances (Peeze Binkhorst et al., 1989A), large vessels were not included in the cross-sectional areas analyzed and the area occupied by other non-fiber cells was small. The larger swelling of the interstitium, as compared to the muscle fibers, might indicate that, as a result of running, the osmotic activity in the interstitium is increased as compared to that in the fibers.

In the animals used for the determination of swelling of muscle tissue (210-290 $\mathrm{g} ; 11-13$ weeks old ), muscle wet weight as well as muscle fiber cross-sectional area increased linearly with increasing body mass. Therefore, muscle wet weight and fiber swelling were corrected for body mass. In the soleus muscle of rat a similar linear correlation between cross-sectional fiber area and age has been reported (Smith et al., 1989).

The intramuscular pressure values, as measured in control rats in this study (1.0 $\pm 0.2 \mathrm{~mm} \mathrm{Hg}$ ), are in agreement with those found in subdermal muscle of the rat by Wiig and colleagues (1981) $(-0.1 \pm 0.7 \mathrm{~mm} \mathrm{Hg})$, who also used micropipettes. Intramuscular pressures measured at rest, using other needle techniques, yield simi- 
lar to slightly higher mean values (1.0-4.4 mm Hg; Hargens et al., 1977; Friden et a.. 1986; Fridén et al., 1988).

Friden and co-workers (1988) found muscle fiber swelling in the anterior tibial muscles of humans, two days after eccentric exercise, to be associated with a limited but significant increase in intramuscular pressure (mean walue $10.5 \mathrm{~mm} \mathrm{Hg}$; rest value $4.4 \mathrm{~mm} \mathrm{Hg)}$ ). This discrepancy between their findings and ours can probably be explained by differences in shape in transwerse section between the anterior tibial muscle and the soleus muscle, as used in the present study. The anterior tibial muscle is almost round whereas the soleus muscle is flat. Because any surface has its smallest circumference in the purely circular form, a muscle which is circular in transverse section at rest in more likely to be compressed within its fascia during swelling than a flat muscle, resulting in more pronounced increases in intramuscular pressure in round than in flat muscles for the same amount of filid increase. Moreover, the anterior tibial muscle is surrounded by bone and very tight connective tissue. It can not be excluded that intramuscular pressure is increased during the first 18 minutes after exercise, but, if so, this temporally increase in pressure cannot explain the focal necrosis observed. Intramuscular pressures of at least 30 $45 \mathrm{~mm} \mathrm{Hg}$ during substantial periods of time are required to cause ischemic muscle damage (Reneman, 1968; Ashton, 1975; Hargens et al., 1977). The time period during which such a high level of intramusclular pressure must be maintained to induce ischemic damage is, under resting conditions, probably many hours (Mäkitie and Teräväinen, 1977; c.f. Matsen, 1975). It is interesting to note that in patients with a chronic anterior compartmental syndrome, at rest intramuscular pressures of about $25 \mathrm{~mm} \mathrm{Hg}$ are found without muscle necrosis (Reneman, 1975). In the present study the intramuscular pressures, as measured between $18 \mathrm{~min}$ and $24 \mathrm{~h}$ after exercise, were far below the pressures required to induce muscle necrosis. Therefore, the muscle fiber degeneration, as observed after exercise, cannot be explained by muscle blood flow disturbances due to increased intramuscular pressure.

Because exercise induced degenerated muscle fibers were situated between apparently unaffected fibers, it could be argued that intramuscular pressure might be increased locally, e.g. in compartments surrounded by endonysia. This is unlikely, because many pressure measurements at different depths and locations along the length of the muscle were made $(n=159)$ and none of these yielded pressure values sufficiently high to explain muscle fiber necrosis. It is also unlikely that elevated pressures could not be measured for technical reasons. Firstly, pressures measured in pressurized isolated muscles were always close to the induced pressure (up to 150 $\mathrm{mm} \mathrm{Hg}$ ), indicating that, even at very high intramuscular pressures, no pressure leakage allong the pipette occurs. Secondly, the tip op the pipette was always located in a previously unaffected part of the muscle, diminishing the possibility of pressure leakage along the tip of the pipette. Finally, measurements made with incisions in the fascia did not yield intramuscular pressure values different from those obtained with direct penetration of the fascia. Tissue damage can probably not be held responsible for the absence of an increase in intramuscular pressure because the small pipette tips used (inner diameter $<1 \mu \mathrm{m}$ ) cause minimal trauma and only 
small free fluid spaces are needed to perform the measurements. It is interesting to note that the servo-nulling counterpressure system is insensitive to osmotic activity of tissue macromolecules (c.f. Wiederhielm, 1981).

It could be argued that the Jength of the anesthetic period might have influenced intramuscular pressure readings. This is unlikely, since no changes in intramuscular pressure were recorded during any of the measuring periods or in rats in which anesthesia was induced during the period of muscle swelling. This is in accordance with the findings of Wiig and colleagues (Wiig et al., 1981) who found no changes in intramuscular pressure during 3-4 hours of anesthesia.

In conclusion, our results show that the rat soleus muscle can swell considerably following exercise without an increase in tissue pressue. The absence of changes in intramuscular pressure after exercise, together with an unchanged post-exercise capillary structure and absence of vascular obstructions (Peeze Binkborst et al., $1989 \mathrm{~A}$ ), indicates that post-exercise perfusion of rat soleus muscle is not impaired as a result of endurance running. Therefore, the exercise induced focal muscle fiber degeneration, that follows muscle swelling in time, is probably not the result of a post-exercise impairment of the microcirculation.

\subsection{SUMMARY}

Exercise induced tissue swelling and its possible consequence for tissue pressure were studied in rat soleus muscle. Rats ran for 75 minutes on a rodent treadmill with a $10^{\circ}$ positive incline. To determine muscle swelling soleus muscles were removed and cryofixed at defined intervals (up to 24 hours) after running. Non-exercised rats served as controls. Wet weights of soleus muscles (corrected for body mass) were increased at 3,6,9 and 24 hours after running as compared to control; the increases being $16 \%, 28 \%, 16 \%$ and $16 \%$, respectively. "The transient increase in muscle wet weight correlated in time with the swelling of the muscle. The increase in muscle volume, as calculated on the basis of fiber and interstitial swelling, was $22 \%, 30 \%$, $22 \%$ and $13 \%$ at $3,6,9$ and 24 hours after running, respectively. Muscle fiber swelling accounted for most of the muscle swelling, but swelling of the interstitium was about 2 times larger than fiber swelling. Degenerated muscle fibers situated between apparently unaffected fibers were first observed at 9 hours after running. Their frequency of occurrence was maximal at the end of the observation period, i.e. 24 hours after running. The muscle swelling was not associated with an increase in intramuscular pressure. Intramuscular pressure, as measured in wivo with a servonulling counterpressure system and glass micropipettes with a tip diameter $<1 \mu \mathrm{m}$, varied between 0.4 and $2.2 \mathrm{~mm} \mathrm{Hg}$ (mean value $1.2 \mathrm{~mm} \mathrm{Hg}$ ). During the post-exercise measuring period ( $18 \mathrm{~min}-24$ hours after exercise), intramuscular pressures of exercised rats did not differ from those in control rats. These findings indicate that increased intramuscular pressure is not responsible for the muscle fiber clegeneration found 24 hours after endurance running. 


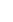




\section{FIBER TYPE RELATED THICK FILAMENT SPACING IN PLASTIC EMBEDDED MUSCLE}

\subsection{INTRODUCTION}

Fast (type I) and slow (type II) twitch muscle fibers can differ in speed and strength of contraction and fatiguability (Garnett et al., 1978; Bodine et al., 1987; Eddinger and Moss, 1987; Metzger and Moss, 1987; Thornell et al., 1987). In addition, their biochemical characteristics can be different (Lowey and Risby, 1971; Whalen et al., 1979; Staron and Pette, 1986; Eddinger and Moss, 1987), and they may use use different metabolic pathways to supply in their energy demand (Peter et al., 1972; Spurway, 1981). Both fiber types can be discriminated in muscle sections using enzymehistochemical (Peter et al., 1972; Dubowitz, 1985), or immunohistochemical (Pierobon-Bormioli et al., 1981; Dodson et al., 1987; Thornell et al., 1987) techniques. Structural and ultrastructural differences between fast and slow twitch fibers have been demonstrated and include size (Andersen and Kroese, 1978; Myrhage, 1978), mitochondrial and lipid content (Staron et al., 1984; c.f. Hoppeler, 1986), and M-line and Z-disc structure (Salmons et al., 1978; Sjöström et el., 1982; Thornell et al., 1987; c.f. Squire, 1981). However, in general there is a considerable overlap between the morphological characteristics of both fiber types (c.f. Hoppeler, 1986), and $\mathrm{M}$-line organization can only be determined in longitudinal sections. It is conceivable that possible morphological differences between contractile fillaments and/or structural proteins, like nebulin, titin, $\alpha$-actinin and M-band proteins (Thornell et al., 1987; Isobe et al., 1988; Wang and Wright, 1988), are associated with the spatial organization of contractile filaments and, consequently, with the functional properties of the muscle fiber. Most studies on myofilament structure and organization have been done on isolated muscle fibers of non-mammalian animals. Studies on mammals are relatively scarce (c.f. Hasselgrove, 1983). In addition, little is known about myofilament structural properties in relation to fiber 
type. This might be due to the technical problems encountered in the use of histochemical fiber typing, while maintaining fibrillar structural organization.

In the present study we investigated the spacing of the thick filament lattice of various fiber types in the soleus, gastrocnemius and peroneus muscles of the rat. The muscles were fixed by perfusion in situ. Muscle fibers in plastic sections were classified using a retrieval method and an antibody specific to type I (slow twitch) fibers for fiber typing. In addition, the absorption of light by fibers in plastic crosssections was studied in relation to their thick filament lattices.

\subsection{MATERIALS AND METHODS}

\section{Animals and procedure}

Male Brown Norwegian rats $(n=13), 11-13$ weeks old and weighing 240-260 g, were used. The animals were housed in a temperature controlled environment (23$26^{\circ} \mathrm{C}$ ) and fed with rat chow (type 1210, Hope Farms) and water ad libitum. They were anesthetized with sodium-pentobarbital (Narcovet; $60 \%$ solution; $1 \mathrm{ml} / \mathrm{kg}$ body weight, i.p.) prior to further handling. All experiments were performed on 5 rats, unless indicated differently.

The procedure used was similar to the one discribed in chapter 4 . After perfusion fixation, muscles were dissected and cut in to small blocks. The blocks were subsequently divided into two parts. One part was embedded in plastic and used for light and electron microscopy. The counterpart was embedded in paraffin and used for immunocytochemical fiber typing. The specificity of the immunocytochemical procedure was tested on cryosections by comparing immunostained cryosections with cryosections stained for myosin ATPase activity. Muscle fibers in plastic sections were classified by comparing them with their immunostained counterparts in paraffin sections. Spacing of thick filaments, light absorption and fiber cross-sectional area were determined in plastic embedded tissue.

Muscle fixation, embedding and sectioning

To preserve the (ultra)structure of the muscles, the left hindlimbs of the rats were chemically fixed by perfusion. After anesthesia and median laparotomy, heparin $(1,000 \mathrm{IU} / \mathrm{kg} \mathrm{b.w.})$ was injected into the liver to prevent blood coagulation. Five minutes after heparin injection, the abdominal aorta was ligated just distal to the renal arteries.

For perfusion of the left hind limb, a silicone cannula (602-155; Silastic; Dow Corning, USA) was inserted into the abdominal aorta and fixed with the tip positioned a few millimeters from the iliac bifurcation. The left hindlimb of each rat was 
fixed in a standard position (ankle $60^{\circ}$, knee $90^{\circ}$, hip $90^{\circ}$ ). The right iliac artery and vein were clamped and the aortic cannula was connected to the perfusion system. Subsequently, the inferior caval wein was incised to ensure unobstructed flow during perfusion. To remove blood from the vascular bed, the hindlimb was initially flushed with buffer ( 2 min at $13.3 \mathrm{kP} ; 0.1 \mathrm{M}$ phosphate buffer, $\mathrm{pH}=7.3$, supplemented with $1.5 \%$ glucose $(\mathrm{D}+) . \mathrm{H}_{2} \mathrm{O}$ w/v and $2.5 \%$ bovine serum albumine). Then the actual fixation was performed through perfusion with fixative (10 min at $13.3 \mathrm{kP}$; fixative solution $4 \%$ paraformaldehyde $\mathrm{v} / \mathrm{v}$ in $0.1 \mathrm{M}$ phosphate buffer with $1.5 \%$ glucose $(\mathrm{D}+) \mathrm{H}_{2} \mathrm{O}$ w/v, $305 \mathrm{mOsm}$ without the fixing agent, $\left.\mathrm{pH}=7.3\right)$. The muscles of the perfused hindlimb appeared to contract during the first seconds of fixation, but the position of the leg was maintained throughout the fixation procedure. Perfusates were prewarmed in a water bath and entered the abdominal aorta at $37^{\circ} \mathrm{C}$. The time between ligation of the abdominal aorta and perfusion witl the fixative ranged from 4-6 minutes.

Fixed soleus, gastrocnemius (deep portion of the lateral head) and peroneus muscles were dissected. Small samples of the muscles were excised and postfixed in the fixative solution for $24-48$ hours at $4^{\circ} \mathrm{C}$. Subsequently, these muscle samples were washed in phosphate buffer with $1.5 \%$ glucose $\left(\mathrm{pH}=7.3,305 \mathrm{mOsm}, 4^{\circ} \mathrm{C}, 15\right.$ min) and carefully cut perpendicularly to the mean muscle fiber orientation into two halves.

One half of each muscle sample was dehydrated through graded ethanol, rinsed in histoclear and embedded in paraffin. The counterpart of this muscle sample was postfixed in $1 \% \mathrm{OsO}_{4}$ in phosphate buffer $\left(4^{\circ} \mathrm{C}, 60 \mathrm{~min}\right)$ and washed three times in phosphate buffer $\left(4^{\circ} \mathrm{C}, 15 \mathrm{~min}\right)$. This part was then dehydrated through graded ethanol, transferred into propyleneoxide and subsequently embedded in Epon-812.

Thin sections $(5 \mu \mathrm{m})$ of paraffin embedded muscle were cut perpendicular to the muscle fiber orientation and used for immunocytochemic muscle fiber typing.

Semithin sections $(1 \mu \mathrm{m})$ of Epon embedded muscle were cut perpendicular to the muscle fiber orientation with glass knives, stained with $1 \% \%$ toluidine blue and investigated under a light microscope (oil immersion objective $40 \mathrm{x} ; \mathrm{N} . A .1 .0$ ). These sections were used for determination of fiber cross-sectional area and light absorption.

Ultrathin sections (about $60 \mathrm{~nm}$ ) were obtained by sectioning perpendicular to the muscle fiber orientation. The sections were contrasted with uranyl acetate $(7 \%$ $\mathrm{w} / \mathrm{v}$ in aqua dest; $10 \mathrm{~min}$ ) and alkaline lead citrate $(5 \mathrm{~min})$. Ultrathin sections were used to study filament spacing by LAED (low angle electron diffraction) and to study the ultrastructural preservation in general and of the areas used for LAED in particular.

Cryofixation was used to preserve myosin ATPase activity of the muscles. After anesthesia muscles were dissected and frozen in melting isopentane.

Cryosections $(10 \mu \mathrm{m})$ were cut perpendicular to the rnean muscle fiber orientation on a cryostat at $-20^{\circ} \mathrm{C}$. Cryosections were subsequently mounted on glass slides, air dried and stored at $-80^{\circ} \mathrm{C}$ until further processing. These sections were used for 
histochemistry, immunocytochemistry and determination of muscle fiber cross-sectional area.

\section{Identification of muscle fiber types}

The monoclonal anti-cardiac myosin IgG (R11D10; Centocor, USA), which cross-reacts with type I muscle fibers, but not with type II muscle fibers (Havenith et al., 1989; Peeze Binkhorst et al., 1989B), was used for immunocytochemical fiber typing. For immunolabeling, paraffin embedded sections $(5 \mu \mathrm{m})$ of muscle tissue were cut perpendicularly to the mean muscle fiber orientation, dewaxed in xylol and transferred to $100 \%$ and $96 \%$ ethanol, respectively. These sections, as well as $10 \mu \mathrm{m}$ sections of cryofixed tissue, were incubated in $0.5 \% \mathrm{H}_{2} \mathrm{O}_{2}$ in methanol ( $15 \mathrm{~min}, \mathrm{RT}$ ), $0.1 \%$ pepsin (Sigma) in $0.1 \mathrm{M} \mathrm{HCl}\left(30 \mathrm{~min}, 37^{\circ} \mathrm{C}\right.$; except cryosections). Subsequently the sections were washed in aqua dest and Tris buffer $(0.1 \mathrm{M}, \mathrm{pH}=7.6, \mathrm{RT})$. The sections were incubated in normal nonimmune rabbit serum (diluted 1:5 in Tris buffer, $10 \mathrm{~min}, \mathrm{RT}$ ) to reduce nonspecific background staining. The sections were then incubated in a moist chamber ( $45 \mathrm{~min}, \mathrm{R} T$ ) with the monoclonal anti-cardiac myosin IgG (R11D10), diluted 1:2000 in Tris buffer/bovine serum albumin (BSA), and washed three times in Tris buffer ( $5 \mathrm{~min}, \mathrm{RT}$ ). Subsequently, sections were incubated with peroxidase-labeled rabbit anti-mouse IgG antibodies diluted 1:200 in Tris buffer/BSA ( $45 \mathrm{~min}, \mathrm{RT}$ ) and rinsed three times in Tris buffer ( $5 \mathrm{~min}, \mathrm{RT}$ ). Peroxidase activity was visualized by incubating the sections in $0.01 \% \mathrm{H}_{2} \mathrm{O}_{2}, 0.05 \%$ diaminobenzidine tetrahydrochloride and $10 \%$ imidazol in Tris buffer ( $5 \mathrm{~min}, \mathrm{RT}$ ). Haematoxylin was used ( 5 min., RT) for nuclear staining.

Using a retrieval method, the fibers in plastic sections were classified by comparing them with their immunostained counterparts in paraffin sections.

The specificity of the antibody for type I fibers was established for each muscle by comparing the immunocytochemical results on cryosections with the serial cryosections stained for myosin ATPase activity. The histochemical staining for myosin ATPase activity was performed at $\mathrm{pH} 9.4$ after preincubation at $\mathrm{pH}=4.3$ or at $\mathrm{pH}=4.6$, allowing discrimination between type I, type ILA, type IIB and type IIC fibers (Dubowitz, 1985).

\section{Determination of filament spacing}

Ultrathin sections were brought into the electronmicroscope and positioned perpendicular to the optical axis. Within each muscle section, groups of two or three fibers of different types were selected at random. LAED diffractograms were obtained from muscle cross-sections, using a selected area diaphragm with a diameter of $20 \mu \mathrm{m}$ and a camera length of $54 \mathrm{~m}$ (Philips CM12 microscope, operating at 100 $\mathrm{kV}$ ). The area of analysis, bounded by the selected area diaphragm, included roughly half a million thick filaments (see result section). The LAED reflections gener- 
ally consisted of two concentric rings, a small and intense one, and a large and less intense one (see Figure 6.2 and result section). The LAED patterns were recorded on negative and 4 times enlarged on photographic prints.

In general, the pattern of the LAED reflections of the filament lattice were elliptical rather than circular. The short and long axis of the elliptical LAED pattern of the filament lattice were measured, using a Kontron MOP-videoplan digitizer system (Zeiss, FRG). Because the long axis of the oval LAED pattern is independent of the angle of sectioning and represents the shortest distance of the lattice spacing, this parameter was used to determine the filament lattice. Filament spacing was calculated according to standard procedures (Beeston et al., 1972). Under our experimental conditions (wavelength of the electrons (lambda) $=0.0037$ $\mathrm{nm}$ and camera length $(\mathrm{L})=54 \mathrm{~m})$ :

$$
\text { lambda } * \mathrm{~L}=\mathrm{R} * \mathrm{D} \quad \Longrightarrow \quad \mathrm{d}=\frac{200}{\mathrm{R}} * 10^{9}
$$

in which $d$ is the filament spacing in $m$ and $R=$ distance between the central spot and the long axis of the diffraction pattern in $\mathrm{m}$.

Assuming a constant radial spacing of the filaments within the sample volume of LAED, the angle between the plane of cutting and the plane perpendicular to the thick filament orientation can be calculated from the long and short axis of the elliptical LAED reflection using:

$$
\text { angle }=\arccos \left({ }^{\text {long axis/short axis }}\right)
$$

\section{Determination of light absorption}

Because it was observed in semithin plastic sections that type I and type II fibers differed in their staining intensity with toluidine blue, the staining intensity of fibers was quantified for all muscles studied. Absorption of monochromatic light (lamb$\mathrm{da}=550 \mathrm{~nm}$ ) by plastic embedded and stained fiber cross-sections was detemined using a circular photocell and a photomultiplier. The same fibers used for LAED were selected. The area of analysis corresponded with a diameter of $12.3 \mu \mathrm{m}$ in the object plane (magnification $\times 26$ ) and was positioned over the central part of each fiber. Parts of the sections containing plastic only (no tissue) were used as a $0 \%$ absorption reference. Covering the light source was used as a total (100\%) absorption reference. Difference in absorption between adjacent fibers was defined as the absorption by type I (in per cent) minus the absorption by (sub)type II fibers (in per cent). 


\section{Muscle fiber cross-sectional area}

Muscle fibers in semi-thin plastic sections stained with toluidine blue and muscle fibers in cryosections stained for ATPase activity were analyzed morphometrically. The cross-sectional area of fibers of different types was estimated using video images of the sections and a Kontron MOP-videoplan digitizer system (Zeiss, FRG).

\section{Statistics}

All results are presented as means \pm S.D. (standard deviation). For statistical analysis a paired Student's $t$ test was used whenever appropriate. For each fiber analyzed, the orientation of the LAED patterns was tested against the expected (average) orientation within single cross-sections, using a Chi-square test. The correlation between light absorption and filament spacing within individual muscle fibers was tested using the calculated coefficient of correlation.

Differences were considered to be statistically significant at p-values $<0.05$.

\subsection{RESULTS}

The (ultra)structure of all muscles at the light as well as the electron microscopic level appeared to be well preserved, as suggested by uncollapsed capillaries and intact mitochondria (see Figures 6.1 and 6.2). In general, no clusters of fixed proteins or blood cells were found in the capillaries, indicating adequate flow through and fixation of all parts of the muscle.

\section{Identification of muscle fiber types}

Staining for ATP-ase allowed discrimination between type I, type IIA, type IIB and type IIC fibers. Type IIC fibers were rare in all muscles studied, hampering further morphometric analysis. Type IIB fibers were absent in soleus muscles. In all muscles tested, the antibody R11D10 labeled only type I fibers, whereas type IIA and IIB fibers were not recognized (data not presented here).

Type $I$ and type II fibers of the soleus, gastrocnemius, and peroneus muscles could easily be identified in plastic sections by comparing them with their paraffin embedded counterpart sections stained with RIIDIO-antibodies.

In the soleus muscle, type I and type II fibers could always be discriminated in the semithin plastic sections because type II fibers had a relatively light sarcoplasm and pronounced subsarcolemmal mitochondrial clusters (see Figure 6.1A).

In plastic sections of the peroneus muscle two clear subpopulations could be distinguished within the group of type II fibers (see Figure 6.1C). One subpopulation 

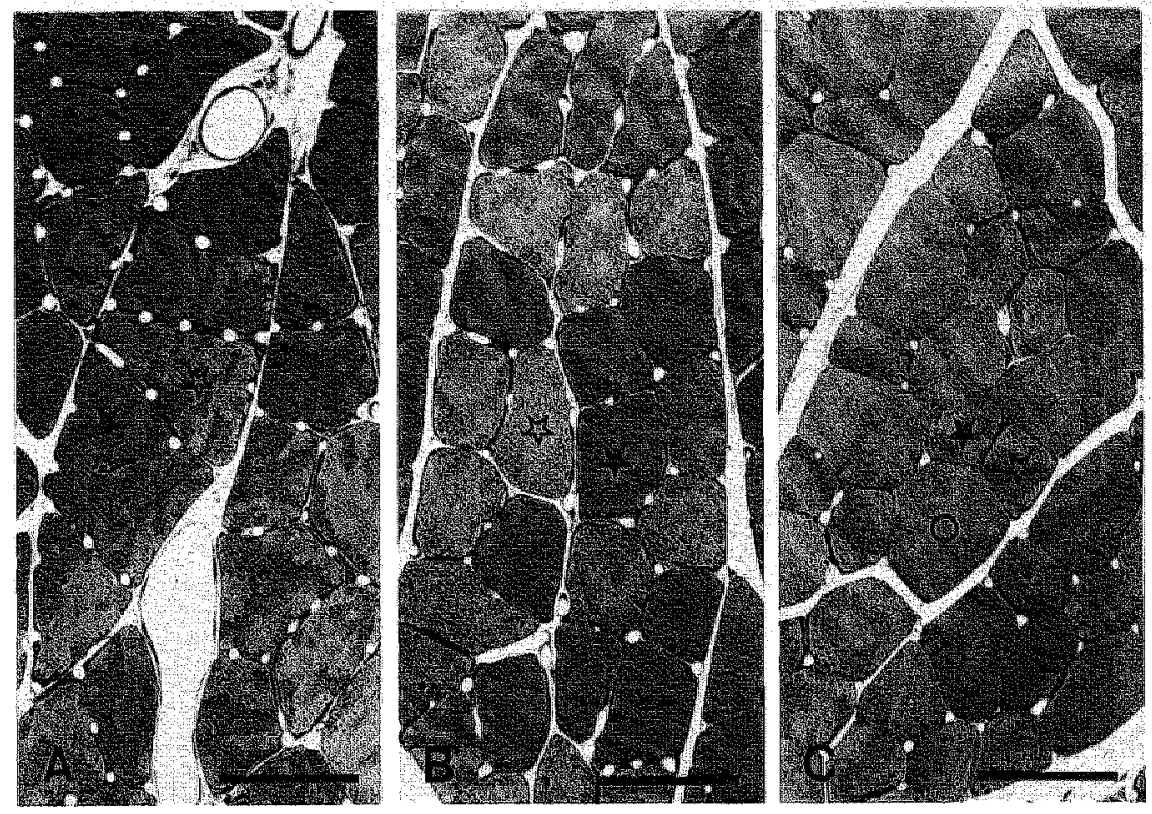

Fig. 6.1. Semi-thin cross-section of soleus (A), gastrocnemius (B) and peroneus (C) muscle, embedded in plastic and stained with toluidine blue. Type I fibers are indicated with a closed asterix, type II fibers with an open asterix. Type II fibers in the peroneus muscle can clearly be separated in to two subgroups. Subgroup II-s, d consist of small fibers with relatively dense cytoplasm and strikingly scattered mitochondria (open asterix). Subgroup II-l,l consists of large fibers with relatively light cytoplasm and no clearly scattered mitochondria (circle). Note the differences in the staining intensity between the different fiber (sub)types. Bars, 50 um.

consisted of small fibers with a relatively dense cytoplasm, many mitochondria scattered throughout the cytoplasm and subsarcolemal mitochondrial clusters. These fibers were provisionally designated as subtype II-small,dark (II-s,d). An other subpopulation consisted of large fibers with a relatively light cytoplasm and some scattered or clustered mitochondria. These fibers were provisionally designated as subtype II-large, light (II-I,I). The same subpopulations of type II fibers could occasionally be distinguished in the gastrocnemius (deep portion of the lateral head), but because the frequency of subtype II-1,l fibers was very low they were not included in the analysis. 


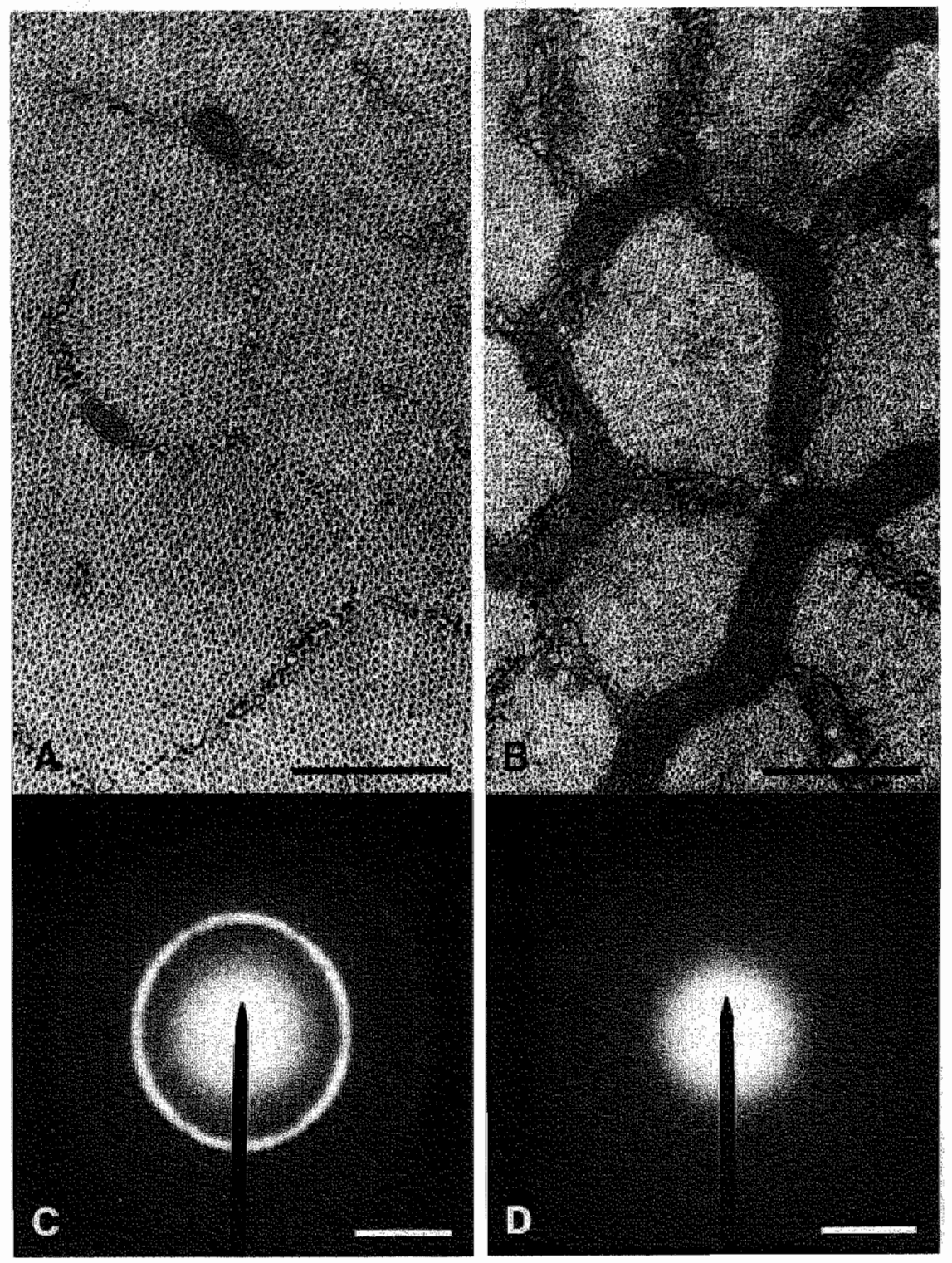

Fig. 6.2. Micrograph of a fiber area containing thick and thin filaments (A) and part of an area of the same fiber containing thin filaments only (B). Note the well preserved fiber utrastructure. LAED patterns of the area with thick fibers (C) show a small intense ring. This ring is absent in the LAED pattern of the area with thin filaments only (D). Bars, $0.5 \mu \mathrm{m}$ (A and B) and $50 \mathrm{~nm}^{-1}$ (C and D). 


\section{LAED pattern of thick and thin filament lattices}

In general, two ellipsoid reflections could be discriminated, a small, intense one and a larger, weak one (see Figure $6.2 \mathrm{~B}$ ). The mean ovalness of the reflections (mean of long axis/short axis ratio) was $0.91 \pm 0.05$ for the soleus, gastrocnemius and peroneus muscles combined and did not differ between any of the muscles or any of the fiber (sub)types. Although the fibers analyzed in each ultrathin section were always closely together (surface area of the sections being $0.01-0.04 \mathrm{~mm}^{2}$ ), the orientation of their filament reflections was not parallel to each other $(p>0.10$; Chisquare test).

The long axis of the low angle, intense reflection corresponded with a lattice spacing of about 30-34 nm, which was similar to the spacing of the thick filament lattice observed in electronmicrographs (see Figure 6.2A). A small but significant $(p<0.05)$ difference in spacing of thick filaments of type I fibers was observed in the soleus, gastrocnemius and peroneus muscles. The long axis of the high angle, weak reflection was observed at a spacing which was $1.74 \pm 0.03(n=23)$ times as large as the low angle, intense reflection. The ratio between the dimensions of the two reflections (1.74) is similar to the theoretical ratio of the linear dimensions of second and first order reflections of a hexagonal lattice (that is $\sqrt{ } 3=1.73$; c.f. Hasselgrove, 1983). Although the high angle reflections might have been the second order reflection of the thick filaments, it was at least in part the first order reflection of the thin filaments, because it was the only distinguishable LAED reflection derived from fiber areas that contained thin filaments only (I-band of the sarcomere; see Figure 6.2).

\section{Thick filament spacing}

Figure 6.3A shows adjacent type I and type II fibers of the gastrocnemius muscle. Figure 6.3B shows the combined LAED pattern of the area indicated in Figure 6.3A. Thick filament spacing in type I and in type II fibers of the different muscles are presented in Table 6.1. In soleus as well as in gastrocnemius and peroneus muscles, the spacing of thick filaments was closer in type I than in (sub)type II fibers. The difference varied between 4 and $9 \%$ (mean $7 \%$ ). Although the differences between the spacing of the thick filaments of type I and (sub)type II fibers were small, these differences were always statistically significant $(p<0.001)$. Thick filament spacing was closer in type I than in type II fibers for $92 \%$ of the fiber pairs compared $(\mathrm{n}=75$; 25 fiber pairs per muscle; see Figure 6.4). 

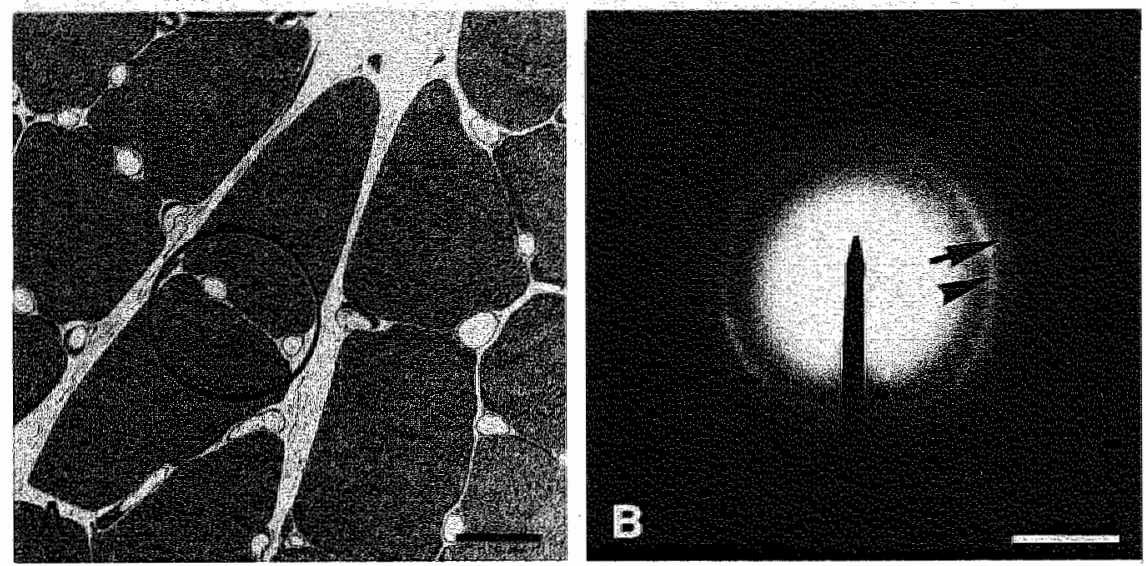

Fig. 6.3. (A) Electron micrograph of adjacent type I (at bottom part of circle) and type II fibers (at top part of circle) of the gastrocnemius muscle. (B) LAED pattern of the area indicated in figure $A$, showing two elliptical diffraction patterns. The high angle elliptical reflection (arrow; small thick spacing) results from the type I fiber, the low angle elliptical reflection (arrowhead; large thick spacing) results from the type II fiber. Bars, $20 \mu \mathrm{m}(A)$ and $50 \mathrm{~nm}^{-1}(B)$.

\section{Light absorption by muscle fiber cross-sections}

Because the staining intensity of the plastic embedded muscle fibers may vary from batch to batch processed and may even vary to some extent within one section, light absorption of adjacent fibers of different types were compared. The results of these absorption measurements are presented in Table 6.2. In all muscles studied, the light absorption by type I fibers was higher than the light absorption by the bordering (sub)type II fibers, although the difference was not always significant. In the soleus muscle the light absorption by type I fibers was $5.4 \%$ higher than the light absorption by type II fibers. In the gastrocnemius muscle, light absorption by type $I$ and type II fibers did not differ significantly. In the peroneus muscle light absorption by subtype II-1, I fibers was 3.4\% and $2.1 \%$ lower than by type I and type II-s, d fibers, respectively. In this muscle light absorption by type II-s,d fibers appeared to be intermediate, although the difference with type I fibers was not significant.

\section{Light absorption and thick filament spacing}

A relatively small spacing of the thick filament lattice correlated with a relatively high light absorption. The correlation between light absorption and thick filament spacing (both parameters expressed as difference to the mean values obtained for 


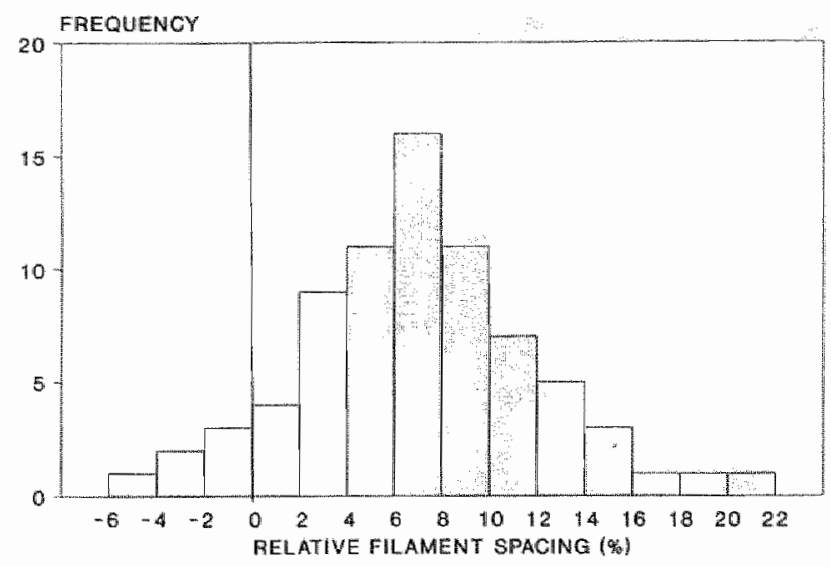

Fig. 6.4. Relative spacing of thick filaments in type II as compared to type Ifibers ((spacing type II-spacing type I)/spacing type I). Combined data for the soleus, gastrocnemius and peroneus muscles. The thick filament spacing in type II is larger than that in type II fibers in $92 \%$ of the cases.

Table 6.1. Thick filament spacing (nm) in cross-sections of muscle fibers.

\section{MUSCLE}

FIBER TYPE

type I

type II

subtype II-s,d

subtype II-1,1

$$
\text { soleus }
$$

$31.2 \pm 1.7$

$30.1 \pm 1.6$

$32.3 \pm 2.1$

gastrocnemius

peroneus
$33.7 \pm 1.9$ *

$33.5 \pm 2.5 *$

Values are mean \pm S.D. for 25 fibers (5 rats). Indicates $p<0.001$ as compared to adjacent type If fibers (paired Student's t test). Spacing in subtype $I I-s, d$ and $I_{\text {- }}$ l, l fibers of peroneus muscles did not differ significantly $(p>0.1)$.

type I fibers in a given section) was highly significant when data for all muscle fibers were combined $(r=0.42 ; n=181$; $p$-value $<0.005)$. The correlation was significant for data obtained from all three muscles tested $(\mathrm{p}<0.005$; r-values being 0.55 $(n=50), 0.42(n=50)$ and $0.39(n=75)$, for soleus, gastrocnemius and peroneus muscle, respectively). 
Table 6.2. Light absorption (absolute difference in percent) by cross-sections of muscle fibers stained with toluidine blue.

\section{MUSCLE}

FIBER TYPE

soleus

gastrocinemius

peroneus

I vs. II

$5.4 \pm 3.7 * *$

$1.3 \pm 4.9$

I vs. II- $-8, \mathrm{~d}$

$-$

$1.4 \pm 3.9$

I ws: III 1,1

$3.4 \pm 4.2 * *$

II-s,d vs. II- 1,1

$2.1 \pm 3.6^{*}$

Values are mean $\pm S . D$. for 25 adjacent fiber pairs (5 rats). * and ** indicate $p<0.05$ and $<0.001$, respectively (paired Student's $t$ test). For all other values of each muscle: $p>0.1$.

\section{Cross-sectional muscle fiber area}

The mean cross-sectional area of muscle fibers, as determined in semi-thin plastic sections, are presented in Table 6.3. In the soleus and the gastrocnemius muscle, the mean cross-sectional areas of type I and type II fibers were similar. In the peroneus muscle the mean cross-sectional areas of subtype II-1,1 fibers was about 2.1 times larger than the other fiber (sub)types, whereas the values for type I and subtype II-s,d did not differ significantly.

In cryosections stained for ATPase activity the muscle fibers of all types were similar in size in the soleus (I and IIA) as well as in the gastrocnemius muscle (I, IIA and IIB). In the peroneus muscle, however, the mean cross-sectional area of type IIB fibers was about 2.5 times larger than that of type II and type IIA fibers ( $n=50$ for each fiber type; 2 rats).

\subsection{DISCUSSION}

The findings in the present study show that at least in the soleus, gastrocnemius and peroneus muscles of the rat, thick filament spacing is closer in type I than in type II fibers (Table 6.1). Although the differences were small (4-9\%), they appeared to be highly significant. A closer spacing of the thick filament lattice corre- 
Table 6.3. Cross-sectional area of muscle fibers $\left(\mu \mathrm{m}^{2}\right)$ embedded in plastic.

\section{MUSCLE}

FIBER TYPE

soleus

gastrocnemius

peroneus

Type 1

$1298+262$

$1112 \pm 308$

$829 \pm 165$

Type II

$1299 \pm 179$

$1078 \pm 140$

subtype II- $\mathrm{s}_{\mathrm{g}} \mathrm{d}$

$823 \pm 153$

subtype $11-1,1$

$1705 \pm 459 *$

Values are mean \pm S.D. for 25 fibers (5 rats). * Indicates $p<0.005$ (unpaired Student's t test) as compared to type I and subtype II-s; d fibers. Only subtype IIl, l fibers of the peroneus muscle are significantly larger than ather fiber types.

lated significantly with a higher light absorption by semithin fiber cross-sections stained with toluidine blue.

In situ fixation of the muscles by perfusion with a paraformaldehyde fixative conserved the ultrastructure as well as the immunoreactivity of the muscle fibers. Classification of muscle fibers in plastic cross-sections was achieved by retrieving their counterparts in immunostained dewaxed sections. Volume changes due to tissue processing were assumed to be similar for all fibers within each muscle sample. By comparing only fibers within individual muscle cross-sections possible differences in the processing between muscle samples were reduced.

Low angle electron diffraction (LAED) allowed the production of diffraction patterns of the relatively large thick filament spacing, as present in muscle crosssections. Filament spacing can also be determined directly from electronmicrographs. However, because $10^{5}-10^{6}$ thick filaments contribute to each LAED pattern (see material and methods), this method is more representative of the entire muscle fiber and less laborious than the measurement of thick filaments spacing on micrographs.

The primary LAED pattern of the thick filament lattice was a ring rather than six individual points as could be expected on the basis of the hexagonal structure of the lattice. Such individual points are found when the lattice is highly regular or only a small area of the lattice is analyzed (e.g. Huxley, 1968). The ring indicates that the thick filament lattice must have a rotational translation within the area of analysis. The LAED pattern of the thick filaments was elliptical rather than circular. The ovalness cannot be the result of skew cutting, tissue compression, orientation of the section in the microscope or astigmatism, because the LAED patterns were oriented 
in all directions within each individual section. Compression, oblique cutting of parallel muscle fibers, oblique orientation of the section in the microscope and astigmatism would all result in a parallel orientation of ellipsoid axes of the LAED patterns, which was not the case. Therefore, the radial asymmetry of thick filament spacing, and probably also of thin filaments in between, might well represent the in vivo situation. The asymmetry might result from a true radial spacing asymmetric of fillaments or from an oblique orientation of the thick filaments within fibers. The functional implications of the radial asymmetry, however, is at present incompletely understood.

The mean spacing of the thick filaments varied between 30 and $34 \mathrm{~nm}$. The difference in spacing of the thick filament lattice of type $\mathrm{I}$, as compared to type II fibers, varied between 4 and $9 \%$ for the three muscle types studied. Differences were small, but were observed in $92 \%$ of the pairs of adjacent type I and type II fibers analyzed. In all muscle fibers analyzed, a higher density of thick filament spacing was generally associated with a higher light absorption by toluidine blue stained cross-sections. In the soleus muscle it was possible to discriminate between type I and type II(A) fibers on the basis of light absorption alone: type I fibers always appeared darker than adjacent type II(A) fibers. The relation indicates that light absorption by the cross-sections was in part due to light absorption by the myofibrils. The relatively low, though significant, coefficient of corre]ation (about 0.45 ) indicates that other factors, e.g. the density of other subcellular structures present in the section, may also have contributed significantly to an increase in light absorption.

Although type IIA and type IIB fibers in cross-sections of the plastic embedded muscle tissue could not be discriminated because the antibody used could not discriminate subpopulations of type II fibers, it is likely that in the peroneus muscle, fibers of the subgroup II-s,d and II-1,I were in general, if not always, type IIA and type IIB fibers, respectively. Arguments in favour of this are:

- The cross-sectional areas of subtype II-s,d as compared to the larger subtype II1,l fibers, was similar to that of type IIA and IIB fibers, respectively.

- Subtype II-s,d fibers, in contrast to subtype II-J, I fibers, showed abundant mitochondria throughout the sarcoplasm and large subsarcolemal mitochondrial clusters. Type IIA fibers have a higher mitochondrial volume than IIB fibers (Staron et al., 1984). In addition, a high oxidative capacity and subsarcolemal clustering of oxidative enzymes is typical for fast-oxidative-glycolytic (FOG) fibers (generally type IIA), in contrast to fast glycolytic (FG) fibers (generally type IIB) (Peter et al., 1972; Pullen, 1977).

Because the spacing of the thick filaments in type II-s, d fibers did not differ from that in II-1,I fibers of the peroneus muscle, it is likely that the same holds for type IIA and IIB fibers of this muscle, respectively.

To the best of our knowledge, no data are as yet available on fiber type related myofilament spacing within the same muscle. The precise causes of fiber type related differences in filament spacing found in this study, and its possible implications for physiological functioning remains unclear at present. 
It could be assumed for the moment that the molecular structures of thick and thin filaments and the structural proteins are largely independent of the fiber type. If so, a larger filament spacing, as in type II fibers as compared to type I fibers, might be ascribed to:

- a smaller sarcomere length at the moment of fixation (Matsubara et al., 1984A; Higuchi and Umazume, 1986).

- a higher electrostatic repulsion between myofilaments due to a high intracellular ionic strength (Matsuda and Podolsky, 1986).

- a higher osmolarity of the sarcoplasm or a lower rigidity of the sarcolemma and/or collagen sheet surrounding the fiber. This can result in relatively more swelling of fibers (c.f. Matsubara et al., 1984A)

- relaxation of type II and contraction of type I fibers. The fiber type related differences in myofilament spacing found in this study (approximately $7 \%$ ) are comparable to differences in filament spacing found between relaxed and isometric contracting skinned skeletal muscle fibers of mouse (6\% decrease; Matsubara et al., 1984A) and frog (3-12\% decrease; Matsubara et al., 1984B). Because the fixative used (paraformaldehyde) is considered to be a reagent for all proteins and is not known for its specific reaction for particular proteins, it is unlikely that it induces contraction in one fiber type (i.e. type I), while an other fiber type (i.e. type II) remains relaxed. Mean differences in the flux of fixative are also unlikely because in the rat soleus muscle the architecture of the microcirculation is fiber type independent (Peeze Binkhorst et al., 1989B). In addition, experiments on contraction and filament spacing have been performed mainly on skinned fibers and it is doubtful that the filament spacing changes during isometric contraction of intact fibers (Hasselgrove and Huxley, 1973).

It is likely, however, that the molecular structures of thick and thin filaments and of their associated structural proteins are different in various fiber types. Biochemical (Lowey and Risby, 1971; Whalen et al., 1979; Eddinger and Moss, 1987) and immunological (Pierobon-Bormioli et al., 1981; Dodson et al., 1987; Thornell et al., 1987) differences between type I and type II fibers have been demonstrated. The myosin heawy chains of fast twitch fibers have a higher molecular weight than those of slow twitch fibers (Staron and Pette, 1986). This finding might indicate that the thick filaments of fast twitch fibers are thicker and/or their cross-bridges are larger as compared to the thick filaments of slow twitch fibers. This could result in the larger spacing of the center of filaments of the fast twitch fibers as was found in this study. Possible structural proteins that might influence or determine thick filament spacing include nebulin, titin, actinin and $\mathrm{M}$-band proteins (Matsubara and Elliot, 1972; Thornell et al., 1987; Isobe et al., 1988; Wang and Wright, 1988; c.f. Maugham and Godt, 1979). Morphology and organization of structural proteins might be fiber type dependent, as is suggested by fiber type related differences in the M-line and Z-disc (Salmons et al., 1978; Sjöström et al., 1982; Thornell et al., 1987), and could be associated with the differences in filament spacing.

It has been established that type II fibers and motor units have a higher maximal shortening velocity and can develop higher tension upon contraction than type I 
fibers and motor units (Henneman and Olson, 1965; Garnett et al., 1978; Bodine et al. 1987; Eddinger and Moss, 1987; Metzger and Moss, 1987). Because a reduction of the myofilament lattice results in a decrease in the maximal shortening velocity (Metzger and Moss, 1987), the low maximal contraction velocity of the type I fibers might well be the result of the small fillament spacing. Although physiological properties like maximal shortening velocity and tension development are probably related to differences in spacing, they might also be related to other molecular differences. Thick and thin filaments of type II fibers might contract faster than those of type I fibers. Muscle fibers with a large filament spacing (i.e. type II) would be expected to develop a relatively small tension per fiber cross-sectional area. In general, type II fibers can develop more force per cross-sectional fiber area than type I fibers (Bodine et al., 1987; Eddinger and Moss, 1987), but the difference can be limited or even absent (Lucas et al., 1987). Because type II fibers have in general a lower relative mitochondrial volume as compared to type I fibers, they might contain a larger amount of contractile filaments per cross-sectional area. In addition, contractile force per thick filament might be larger in type II as compared to type I fibers. A large filament spacing in type II fibers might then still be compatible with a high contractile force per fiber cross-sectional area.

It was found in this study that thick filament lattice spacing and, to a lesser extent, light absorption can contribute to the identification of type I and type II fibers in sections of plastic embedded muscle. Moreover, the results obtained indicate that the physiological characteristics of slow and fast twitch fibers are reflected in spacing of the contractile filaments. It is likely that the difference in filament spacing are the result of fiber type related differences in molecular architecture.

\subsection{SUMMARY}

In the present study the possible relation between muscle fiber type and thick filament spacing was investigated in the soleus, gastrocnemius and peroneus muscles of the rat. All muscles were fixed by perfusion with the hind limb of the rats fixed in a standard position. Filament spacing was determined in ultrathin cross-sections of plastic embedded muscle tissue using Low Angle Electron Diffraction. Fibers in sections of plastic embedded muscle were classified by comparing them with their immunolabeled counterparts in sections of paraffin embedded tissue. In the soleus, gastrocnemius and peroneus muscles, the spacing of thick filaments in type I fibers was 31,30 and $32 \mathrm{~nm}$, respectively. In all muscles studied, the thick filament spacing was significantly لarger in type II (fast twitch) fibers than in type I (slow twitch) fibers, the differences ranging between 4 and $9 \%$. Large spacing of the thick filaments was associated with low light absorption by the same fibers in semithin plastic cross-sections stained with toluidine blue. 


\subsection{APPENDIX}

The LAED reflection of cross-sections of the M-band of muscle fibers yielded a small, intense reflection and a large weak reflection (see Figure 6.2C). The high angle reflection might be in part the second order reflections of the thick filament lattice because they were $\sqrt{ } 3$ times as large as the low angle reflections (Hasselgrove, 1983). However, because the high angle reflection was also present in regions containing only thin filaments (I-band of the sarcomere; see Figure 6.2D), it was probably also in part the first order reflection of the thin filaments. This is in accordance with the expected value of the first order reflection of thin filaments. Figure 6.5 shows a lattice unit cell bounded by four adjacent thick filaments, enclosing two thin filaments. If the distance between the thick filaments is $d$, than the distance between the thin filaments will be $d / \sqrt{ } 3$. According to formula 1 , this indicates that the first order reflection of the thin filaments will be $\sqrt{ } 3$ times as large as the first order reflection of the thick filaments.
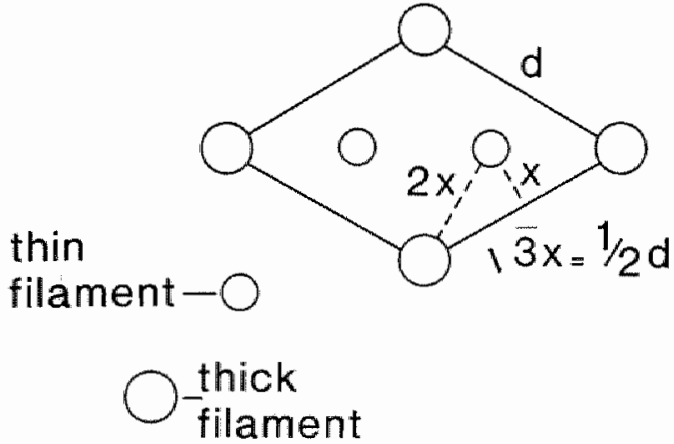

Fig. 6.5. Unit cell of thick filaments. Two thin filaments are enclosed in each cell. The spacing of the thin fillaments is $1 / \sqrt{ } 3$ times as large as the spacing of the thick filaments $(d)$.

The high angle reflection of the thin filaments in the I-band of the sarcomere was $14 \%$ smaller than the low angle reflection of the thin filaments in the M-band (see Figure 6.2C and 6.2D). In the $\mathrm{M}$-band, thin filaments are located in the center of the triangles formed by the thick filaments (see Figure 6.2A). Each triangle has an surface area of

$$
1 / 2 * 1 / 2 \vee 3 * d * d=1 / 4 \sqrt{ } 3 d^{2}
$$

In regions without thick filaments, that is in the I-band and near the $\mathrm{Z}$-line, thin filaments are arranged in squares (see Figure 6.2B). Each square must have the same area as the triangle, because each square encloses also $1\left(=1 / 4^{*} 4\right)$ thin fila- 
ment. Therefore, the length of the sides of the squares, that is equal to the thin filament spacing in the I-band, must be

$$
\sqrt{1 / 4 \sqrt{ } 3 * d^{2}}=1 / 2 * \sqrt[4]{ } 3 * d
$$

Consequently, because the spacing of the thin filaments is $\mathrm{d} / 3$ in the $\mathrm{M}$-band, the spacing of the thin filaments in the I-band is increased with a factor

$$
1 / 2(\sqrt{ } \sqrt{3}) * \sqrt{ } 3
$$

that is about $14 \%$. According to formula 1 , the reflection of the thin filaments near the I-band must therefore decrease by the same factor as compared to the reflection of thin filaments in the M-band, which is in accordance with our findings. 


\section{FLUORESCENT LABELING OF CAPILLARY ENDOTHELIUM IN VIVO}

\subsection{INTRODUCTION}

Visualization in vivo of capillaries in striated muscle is difficult. The low contrast between the endothelial cells and surrounding tissues in combination with the thickness of the overlying structures hampers visualization. Therefore, capillaries are usually studied in thin muscles (Eriksson and Myrhage, 1972; Myrhage and Hudlicka, 1976; Damon and Duling, 1984; Wiederhielm and Slaaf, 1987). However, even in these preparations capillaries may be missed (Damon and Duling, 1984).

Fluorescent labeling of the endothelial cells would improve contrast of the capillary wall, and hence facilitate its recognition. This will improve assessment of capillary diameter. In addition, labeling of endothelial cells and/or their glycocallyx in vivo may allow investigation of intraluminal protrusion of the cells (Weigelt et al.; 1981; Tyml et al., 1982) or fluctuations in the thickness of their glycocalyx (Desjardin and Duling, 1988).

To the best of our knowledge, no fluorochromes are known that 1) accumulate specifically on or in endothelial cells, 2) do not leak out of the circulation rapidly, and 3) do not cause unwanted side effects. Substances like acridin orange, acridin red, pyronin B and mepacrine can label the wall of mesenteric microvessels in vivo (Tangelder et al., 1982), but it is unknown whether they will label capillary endothelial cells in striated muscle without causing a high background fluorescence. Endothelial muclei can be labeled in vino with Bis-benzamide $H 3342$ (Reinhold and Visser, 1983) and the huminal side of especially venular blood vessels can locally be labeled with fibrinogen and fibronectin tagged to fluorescein-isothiocyanate (Witte, 1983), but with these substances only partial labeling of the endothelial layer is achieved.

Several fluorochromes are known which can label endothelial cells in vitro. Cultured endothelial cells can be labeled specifically with acetylated low density lipo- 
protein conjugated to a fluorescent probe. The substance is selectively taken up by the endothelial cells and the fluorescent probe accumulates in the lysosomal membranes (Voyta et al., 1984). Lectins have been used to label endothelial cytoplasm and/or their outer membrane in vitro (Holthöfer et al., 1982 „Simionescu et al., 1982; Ninomiya et al., 1985; Little et al., 1986). Thioflavin-S labels the vascular intima and has been studied in tissue sections after perfusion with the dye (Schlegel, 1949; Fourman and Moffat, 1967; Dohrmann and Wick, 1971). Because the surface of endothelial cells is negatively charged, (poly)cations, such as polylysin, might accumulate on the capillary inner membrane in vivo (Simionescu et al., 1981A and 1981B).

In this study, a series of fluorescent dyes was tested in the rat for their ability to label capillary endothelium in vivo. Labeling of capillaries was screened in mesentery, using intravital fluorescence microscopy. In addition, the fluorochromes pyronin $\mathrm{B}$, thioflavin-S and acridine orange were tested in the soleus muscle.

\subsection{MATERIALS AND METHODS}

\section{Animals and tissue preparation}

Male Brown Norwegian or Wistar rats, 11-15 weeks old and weighing 250-330 g, were used. The animals were housed in a temperature controlled environment (23$26^{\circ} \mathrm{C}$ ) and fed with rat chow (type 1210, Hope Farms) and water ad libitum.

The rats were anesthetized with sodium-pentobarbital (60\% solution (Narcovet); $1 \mathrm{ml} / \mathrm{kg}$ body weight, i.p.). The animals were then tracheotomized and intubated to facilitate breathing. The right femoral artery was cannulated to measure arterial blood pressure, using an external pressure transducer (CTC, CP-01). The right femoral veim was cannulated for injection of dyes. Additional anesthesia was given whenever necessary.

\section{Mesentery preparation:}

Through a lateral incision in the abdomen, the coecum and distal ileum were exposed. Care was taken to minimize trauma. The second module of the mesentery, counting from the coecum, was spread over a siliconized glass plate mounted in an electrically heated microscope table $\left(37^{\circ} \mathrm{C}\right)$ and the intestine was kept moist with overlying wet gauze. The preparation was continuously superfused with buffered Tyrode solution gassed with $95 \% \mathrm{~N}_{2} / 5 \% \mathrm{CO}_{2}\left(37^{\circ} \mathrm{C} ; \mathrm{pH} 7.4\right)$. Whenever damaged vessels were observed, the next module of the mesentery was prepared for observation. Only preparations with a limited number of sticking or rolling leukocytes in the venules were used for analysis. 
Soleus muscle preparation:

The animal was placed in a prostrate position. The foot of the left hind limb was fixed to minimize movement. The dorsal side of the skin of the lower hind limb was incised. The gastrocnemius muscle, overlying the soleus muscle, was carefully split, causing as little trauma as possible. The two halves were pulled aside with sutures (Ethibond 6-0) untill the soleus muscle became accessible. The connective tissue overlying the soleus was carefully removed. Subsequently, the animal was placed under the microscope. The tissue was kept moist by covering it with wet gauze, except for the part under observation which was continuously superfused with the same Tyrode solution used for the mesentery preparation. Only undamaged vessels that showed normal blood flow were used for analysis.

\section{Microscope and recording system}

The microscope and recording system were the same as previously described in detail (Tangelder et al., 1982). In short, all observations were made with a Leitz intravital microscope adapted to telescopic imaging (Slaafet al ", 1982). For bright field microscopy of the mesentery preparation, transillumination with a tungsten lamp was used. Incident illumination of the soleus muscle was performed using a Xenon (150W) or Mercury (100W) arc. Fluorescence microscopy was performed with an incident fluorescence illuminator (Leitz Ploemopak 2.2; tube factor 1.25 or 1.6), using a $\times 50$ water-immersion objective (SW50; numerical aperture 1.00). The filter combinations employed with each dye are listed in Table 7.1. In addition, each dye was tested using the N2.1, 12 and D filter combinations to check whether its fluorescence could be improved. The tissues were directly observed with a x10 ocular. Images were recorded on tape using a sensitive video camera (SIT, Bosch) and a video recorder (Sony, Betamax). Final magnification at the front plane of the T.V. camera was $\times 78$ or $\times 100$, depending on the tube factor of the Ploemopak.

To obtain insight into the fluid dynamic effects of pyronin $B$ (see results), changes in mean red blood cell velocity ( $\mathrm{rbc}$ ) and in wascular diameter were determined in arterioles of the mesentery preparation before and after injection of this dye. Mean Vrbc was measured on-line using the dual-slit photometric technique (Wayland and Johnson, 1967). The dual-slit light rods were positioned at the center of the image of the blood vessel. Changes in local arteriolar diameter were measured from video images, using a shearing monitor device (Intaglietta and Tompkins, 1973).

Administration of fluorescent compounds and enzyme substrates

All dyes and enzyme substrates used and their commercial source are presented in Table 7.1. A conjugate of poly-1-lysin (PLL; M =230,000; Sigma) and fluorescein 
Table 7.1. Fluorochromes, fluorogenic enzyme substrates and FITC-Jabeled lectins, their commercial sources and fiffer sets used.

Dye

Source

Filter

\section{Fluorochromes}

Acridin Yellow

Acridin Orange

Acridine Red

Acriflawin

Alizarin Red S

Bovine Serum Albumin (BSA)-FITC

Coriphosphin

Coumarin 440

Coumarin 522

Cresyl violet 670 perchlorate

Evans Blue

FITC isomere I

Fluorescein-sodium

Goat-anti-rabbit-FITC

Kinton Red 620

LD 490

4-methyl-umbelliferon

Neutralrot rein

Poly-1-lysin-FITC

Pyronin B

Rhodamin B isoth.

Rhodamin IgG

Rhodamine B

Rivanol

Rose Bengal

Safranin 0

Thiazin Red R

Thioflavin-S

TRITC

Xylenol Orange Tetrasodium

$\begin{array}{ll}\text { Chroma } & 2 \\ \text { Chroma } & 2 \\ \text { Chroma } & 1 \\ \text { Chroma } & 5 \\ \text { Eastman Kodak } & 1(2) \\ \text { Sigma } & 2 \\ \text { Chroma } & 2 \\ \text { Exciton } & 3 \\ \text { Exciton } & 3(2) \\ \text { Exciton } & 1 \\ \text { Pfaltz \& Bauer } & 1 \\ \text { Sigma } & 2 \\ \text { Pfaltz \& Bauer } & 2 \\ \text { Sambio } & 2 \\ \text { Exciton } & 1 \\ \text { Exciton } & 3 \\ \text { Serva } & 4 \\ \text { Chroma } & 1 \\ \text { Home made } & 2 \\ \text { Sigma } & 1 \\ \text { Pfaltz \& Bauer } & 1 \\ \text { Brunschwig } & 1 \\ \text { Pfaltz \& Bauer } & 1 \\ \text { Pfaltz \& Bauer } & 3 \\ \text { Pfaltz \& Bauer } & 1 \\ \text { Eastman Kodak } & 1(2) \\ \text { Serva } & 1 \\ \text { Sigma } & 3(2) \\ \text { Sigma } & 1 \\ \text { Eastman Kodak } & 1\end{array}$

Enzyrne substates

Dil-Ac-LDL*

2-4 methyl-umbelliferylphosphate

3.0 methyl fluorescein phosphate

Methyl-umbelliferyl-phosphate -galactosamide

BTI

Serva

Sigma

Serva

E-Y Labs

E.Y Labs

E-Y Labs

E. Y Labs 
isothyocyanate (FITC) was prepared as follows. PLL (150 mg) was added to an FITC solution (6.35 mg dissolved in $0.15 \mathrm{M} \mathrm{Na}_{2} \mathrm{HPO}_{4} ; \mathrm{pH}$ 9.2). The mixture was stirred for 1 hour at room temperature. To remove unconjugated FTrC the conjugated sample was passed over a Sephadex 25 fine column $(40 \times 2 \mathrm{~cm})$ equilibrated with phosphate buffered saline ( $\mathrm{pH}$ 7.2). Concentrations of FITC and PLL, were determined by measuring the extinction in $1 \mathrm{~cm}$ cuvettes at 495 and $280 \mathrm{~nm}$ wavelength, respectively. Eluted fractions with molecular FITC/PLL ratios $>1$ and $<4$ were pooled and freezedried.

Each dye used was tested in at least one rat. Ten mg of a dye was dissolved in 1 $\mathrm{ml}$ of physiological saline at room temperature by vigorous shaking. The solution was subsequently centrifugated to remove undissolved dye particles and the supernatant was isolated. If the substances could not be dissolved adequately in this way, $10 \mathrm{mg}$ were also dissolved in $1 \mathrm{ml}$ of $96 \%$ ethanol, using the same procedure. The ethanolic solution was subsequently diluted $1: 1$ in saline.

Each dye was tested in vivo, at first in the mesenteric microvessels. Under continuous microscopic observation, $0.1,0.2$ and $0.6 \mathrm{ml}$ of a saline solution were injected intravenously at about 1 min intervals, until weak fluorescence of the blood plasma became visible. If no accumulation of the dye in or on the endothelial cells was observed within $3 \mathrm{~min}$ (within $15 \mathrm{~min}$ for FITC labeled macromolecules and enzyme substrates) or when the fluorescence faded, an additional bolus was given. Whenever appropriate, $0.1,0.2$ and $0.4 \mathrm{ml}$ of the ethanolic/saline solution $(0.6 \mathrm{ml}$ was usually fatal) of the same dye was tested as well. pyronin B, thioflavin-S and acridine orange were tested in a similar way (see results) in the soleus muscle. Because accumulation of acetylated low density lipoprotein (Ac-LDL) labeled with DiI (1,1'-dioctadecyl-3,3,3',3'-tetramathyl-indocarbocyanine perchlorate) takes several hours (Voyta et al., 1984), this probe (DiI-Ac-LDL; $0.2 \mathrm{ml} ; 200 \mu \mathrm{g} / \mathrm{ml}$ ) was injected into the tail artery of a rat $2 \mathrm{~h}$ and $30 \mathrm{~min}$ prior to preparation of the mesentery. The mesentery preparation itself took another $30 \mathrm{~min}$. An additional bolus of

Table 7.1. (Continued)

Leitz interchangeable filter sets:

1: No. N2,1 (excitation filter: BP 515-560, dichroic mirror: RKP 580, barrier filter: $L P$ 580)

2: No. I2 (BP 450-490, RKP 510, LP 515)

3: No. D (BP 355-425, RKP 455, LP 460)

Combination of Leitz $3 \mathrm{~mm}$ excitation filler, dichroic mirror and barrier filter:

4: UG1, TK 400, K430

5: $B G 12, T K 455, K 490$

Dil-Ac-LDL $=$ Acetylated Low Density Lipoprotein labeled with 1,1'-dioctadecyl-1-3,3,3',3'-tetramethyl-indo-carbocyanine perchlorate. BTI =Biomedical Technologies Inc. 
$0.3 \mathrm{ml}$ Dil-Ac-LDL was given $30 \mathrm{~min}$ after preparation via the femoral wein catheter.

\section{Evaluation of fluorescent labeling in vivo}

About 10-20 s after intravenous injection, the lumen of the vessels became fluorescent in the case of most dyes, the intensity of the fluorescence being dependent on the amount of dye injected. The dye could either stay within the vessels for a long period of time, sometimes more than $1 / 2 \mathrm{~h}$, or slowely or more rapidly leak out. In the case of leakage, fluorescence of the vascular lumen faded, while that of the surrounding tissue increased. Whether labeling of blood plasma, endothelial cells, leukocytes and thrombocytes and leakage of the dye out of the microvessels occured was determined by two persons, directly via the microscope and/or from a T.V. monitor. Preferential labeling of a structure was considered as positive if, according to both observers, the fluorescence of the structure was clearly above the background level. Failure to label a structure could indicate that the dye did not attach to the structure or that its fluorescence was masked by the fluorescence of its background, i.e. blood plasma and/or the mesentery itself. Labeling of the cytoplasm, membrane and/or nuclei of capillary endothelial cells in the mesentery was checked by combining the fluorescent image (epi-illumination) with trans-illumination.

\subsection{RESULTS}

In-vivo labeling in the mesentery

The labeling of structures in mesenteric microvessels by the injected fluorescent dyes is summarized in Table 7.2.

Some dyes injected did not leak out of the vascular bed and labeled the vascular lumen for extended periods of time, i.e. more than 1/2 h. As might be expected, these dyes included the large FITC-protein conjugates like BSA-FITC, goat-antirabbit-FITC, PLL-FITC and all FITC labeled lectins as well as rhodamin IgG. However, some of the dyes with a relatively low molecular weight, e.g. evans blue, fluorescein-sodium and thioflavin-S, labeled the vascular lumen as well.

When leakage occurred, the fluorescence of the vascular lumen faded and often structures could be seen that could not be detected before.

Many dyes accumulated in or on structures to such an extent that the background fluorescence of the blood plasma and mesenteric tissue could be overcome. Pyronin-B, neutralrot rein and acriflavin labeled the endothelial cytoplasm (and nuclei) satisfactorily. However, neutralrot rein and acriflavin leaked out of the circulation rapidly, making them potentially unsuitable for endothelial labeling and 
visualization of capillaries in thicker tissues such as skeletal muscle. Patchy labeling of endothelial cells was obtained with acridine red, BSA-FITC,PLL-FITC and Safranin-O. Acridin yellow was found to be a relatively specific fluorescent label for nuclei of endothelium and leukocytes. Several dyes, including most lectins conjugated with FITC, labeled thrombocytes.

Of all fluorogenic enzyme substrates, only 2-4-methyl-umbelliferyliphosphate was hydrolyzed and yielded a fluorescent compound, not inside the endothelium but probably in the blood plasma. The other three enzyme substrates tested did not label any of the structures investigated and were probably not hydrolyzed, although one of them was also a substrate for phosphatases like the 2-4 methyl umbelliferylphosphate which was hydrolyzed in the blood. Also, no fluorescence could be detected in the mesenteric microvessels following injection of coumarin 440 and coumarin 522, although fluorescence of the solutions of both dyes was observed $i n$ vitro. The solubility in saline of both coumarins was low. Both dyes dissolved partially in ethanol ( $96 \%$ ), but might have precipitated when injected into the animals.

\section{In-vivo labeling in the soleus muscle}

Acridine orange, thioflavin-S and pyronin $B$ were tested in the soleus muscle preparation. Acridine orange labeled leukocytes and nuclei of the endothelium, but leaked out of the circulation rapidly causing a high background fluorescence. Thioflavin-S labeled the capillary lumen, but did not label the endothelium at any of the doses tested. Pyronin B labeled the capillary wall and did not leak from the circulation rapidly. Therefore, the effects of this dye was studied in more detail.

\section{Labeling of endothelium with pyronin B}

The best results with pyronin B were obtained when the capillary wall was loaded with a high dose of the dye and fluorescence was maintained thereafter with smaller bolus doses. For these investigations, pyronin B was completely dissolved in saline $(2-4 \mathrm{mg} / \mathrm{ml})$. When capillaries in the mesentery or soleus muscle were observed, the following sequence of events occurred. About 10 seconds after injection of $0.5-1 \mathrm{mg}$ (mesentery) or 1-2 $\mathrm{mg}$ (soleus) pyronin B, the dye became visible. During the first 10-15 seconds thereafter the whole vessel was brightly fluorescent. Then plasma fluorescence diminished rapidly and the endothelial cytoplasm and nuclei became visible (Figures 7.1B and 7.2). By combining epi- and trans-illumination of the mesenteric microvessels, it was observed that the fluorescent layer was wider than the endothelial cells. This indicates that besides the endothelial cytoplasm and nuclei pyronin B might label the endothelial glycocalyx and basal membrane. During 2-3 minutes the brightness was sufficient for detailed observation. If thereafter the brightness of the capillary wall was maintained by bolus injections of $0.2 \mathrm{mg}$ 
Table 7.2. Labeling of structures in mesenteric microvessels by fuorescent dyes. Indicatted are leakage of the dye out of the vessels and labeling of endothelial cytoplasm (Cyto) and nuclei. leukocytes (theuko) and thrombocytes (Thrombo).

Dye

Labcing

Lumen Endothelium
cyto neviciei

Fluorochromes

Acridin Yellow

Acridine Red

Acrillaviry

Alizarin Red si

Bowine Serum Alburmin (BSA)-FITC

cororiphosphis

colt Coumarin 440

colf Coumarin 522

Cresyl violet 670

perchlonte.

Evans Blie

FITC isomere 1

Filuorescein-sodium

Gont-anti-rabbit-Fl'TC

Kiton Red 620

$\infty \mathrm{LD} 490$

cot methyl- ambelliferon

Neutral rot rein

Poly-1-Jysin-FITC

Pyronin $\mathbb{B}$

colRlaodamin E isoth.

Rhodamin IgC

Rhodamine $B$

Rimanol

Rose Bengal

Safranin 0

Thiazin Red $\mathrm{R}$

Thioflatin-s

TRITC

Xylenol $O$

\section{Enzume substates}

HDiT-AC-LDL

\section{2*4-methyl}

unbelliferylphosphto

"1 3 - 0 methyl fluorescein

phosphate

ont Meithyl-umbelliferyl.

phosphate-galactosemnide

\section{Lewtins}

Conctanaralin A (ComA)

FITC

Riccinus Communis

Agslutimat (RCA)-FTC

Wlex Europacus

AgElutinin (UEA) FITC

Wheat Germ Agglaumin

(WOA) FITC

\begin{tabular}{|c|c|c|c|}
\hline - & $=$ & 4 & ++ \\
\hline . & $*$ & 童 & + \\
\hline- & \pm & + & 4 \\
\hline . & - & . & $=$ \\
\hline+ & * & $=$ & - \\
\hline . & - & - & + \\
\hline n & • & . & , \\
\hline . & . & • & . \\
\hline - & - & + & + \\
\hline+ & - & - & - \\
\hline \pm & $\infty$ & $\sim$ & - \\
\hline \pm & - & " & 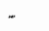 \\
\hline+ & - & - & - \\
\hline \pm & $=$ & n & - \\
\hline+ & - & - & + \\
\hline - & - & - & - \\
\hline- & + & +4 & 4 \\
\hline+ & 诲 & - & + \\
\hline \pm & + & ++ & $\pi$ \\
\hline+ & - & - & $\sim$ \\
\hline+ & - & - & - \\
\hline * & 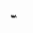 & - & - \\
\hline - & - & - & - \\
\hline- & - & . & $*$ \\
\hline$\infty$ & $*$ & + & - \\
\hline - & - & - & - \\
\hline+ & 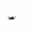 & . & - \\
\hline \pm & $=$ & - & $=$ \\
\hline+ & . & - & - \\
\hline
\end{tabular}

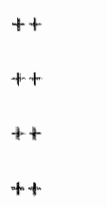

$+t$

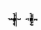

$+t$

ta. 
(mesentery) or $0.5 \mathrm{mg}$ (soleus) pyronin B every 3 minutes, the endothelial cells could be observed in the mesentery for about $40 \mathrm{~min}$ and in the soleus muscle for about $20 \mathrm{~min}$ before leakage of the dye into the tissue increased background fluorescence to unacceptably high levels. In the soleus muscle (mesentery not tested) brightness of the capilary wall could also be maintained by continuous infusion of $4-8 \mathrm{mg}$ pyronin $\mathrm{B} /$ hour for about $20 \mathrm{~min}_{\text {. }}$

\section{Side effects of dyes}

The effect of intravenous injection of pyronin B on arterial blood pressure, red blood cell velocity in mesenteric microvessels ( $\mathrm{Vrbc}$ ) and arteriolar diameter was investigated in three rats, using trans-illumination alone. Bolus injections of $0.5,0.2$, $0.2,0.2$ and $0.2 \mathrm{mg}$ pyronin $B(4 \mathrm{mg} / \mathrm{ml})$ were administered at three minutes intervals. Arterial pressure and Vrbc decreased dramatically after these injections. Within seconds after the first injection $(0.5 \mathrm{mg})$, both arterial blood pressure and Vrbc increased slightly (to 128 and $115 \%$ of the initial value, respectively) and dropped within $1 \frac{1}{2}$ minute to about $58 \%$ and $37 \%$ of the initial value, respectively. At the time of the second bolus, arterial blood pressure had increased to $62 \%$ and $\mathrm{Vrbc}$ to $74 \%$ of the initial value, respectively. The effects of the subsequent bolus injections to maintain the fluorescence ( 4 times $0.2 \mathrm{mg}$ ) were similar, but less pronounced and overall arterial blood pressure and Vrbc increased gradually after the first bolus. Arterial blood pressure and Vrbe returned to control values about 10 and 20 minutes after the last bolus injection, respectively. The mesenteric arteriolar diameter did not change as a result of the injections of pyronin $B$.

\section{Table 7.2 (continued)}

Labeling of lumen:

$++=$ no noticeable background fluorescence in mesenteric tissue after $>30$ $\min$.

$+\quad=$ relatively little fluorescence background

$\pm \quad=$ background fluorescence increases slowly with time (minutes)

- $\quad=$ background fluorescence increases almost immediately (seconds)

Labeling of other structures:

,,+++ \pm and $-:$ decreasing fluorescence as compared to background, i.e. blood plasma and mesenteric tissue. The relative number of leukocytes and thrombocytes labeled was not determined.

$\infty$ indicates that dye was dissolved in ethanol and diluted 1:1 with saline. \# indicates that no fluorescence of blood plasma or any other structure could be obtained with the experimental protocol used.

* indicates that the endothelial inner membrane is locally and patchy labeled. 

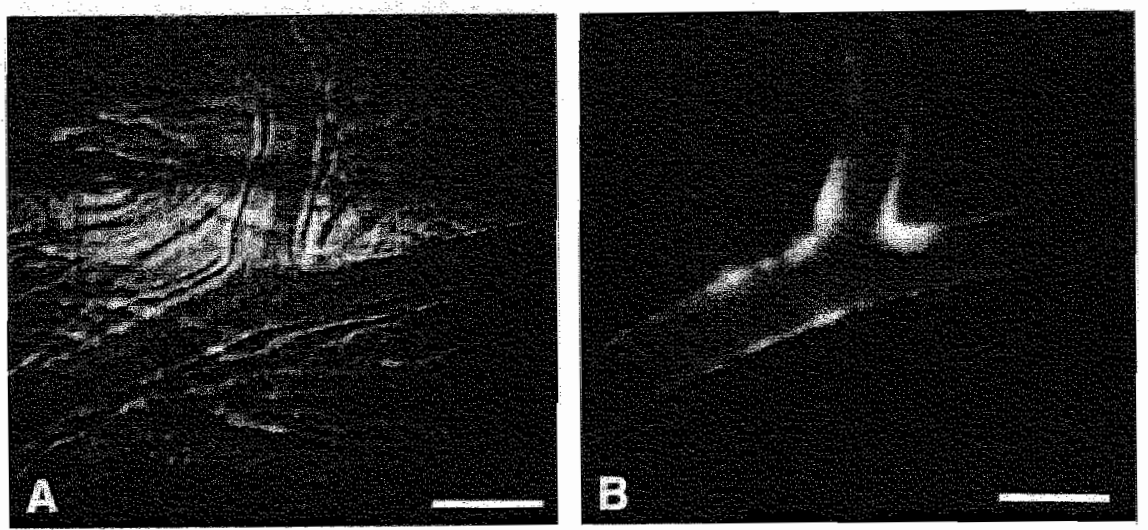

Fig. 7.1. Labeling of the wall of rat mesenteric microvessels by pyronin $B$. (A) Trans-illumination: the outline of the microvessels is clearly vissible. (B) Epi-illumination of the same vessels. Bar, $20 \mu \mathrm{m}$.

Epi-illumination of microvessels after labeling of the blood plasma with acridin yellow, acriflavin, PLL-FTTC, safranin O, rose bengal and thioflavin-S caused thrombus formation at the site of irradiation, resulting in partial or total inhibition of blood flow within seconds or minutes. In general, non-illuminated capillaries did not show these effects, but thrombus formation could be induced by illuminating these vessels as well. Especially thioflavin-S, and rose bengal appeared to be potent inductors of thrombus formation upon irradiation. PLL-FITC caused sticking of individual platelets or small platelet aggregates to the capillary wall, in general during short periods of time.

Injection of $10 \mathrm{mg}$ PLL-FIrTC or $10 \mathrm{mg}$ pyronin B was fatal (1 rat tested for each dye). Thioflavin-S caused respiratory problems in the animals tested. Choriphosphin caused the death of the animal after the last bolus injection $(0.4 \mathrm{ml}$ saturated in saline/ethanol), which might in part have been the result of the ethanol.

\subsection{DISCUSSION}

In the present study the possibility to label endothelial cells in vivo by fluorescent dyes was investigated. An important objective was to improve visualization of capillaries in opaque tissue, such as skeletal muscle. To find fluorescent labels for the capillary wall, fluorescent dyes and fluorogenic enzyme substrates were initially studied in the rat mesentery, because the mesentery is thin and transparant and microvessels can be visualized in greater detail, as compared to capillaries in skeletal muscle preparations. 


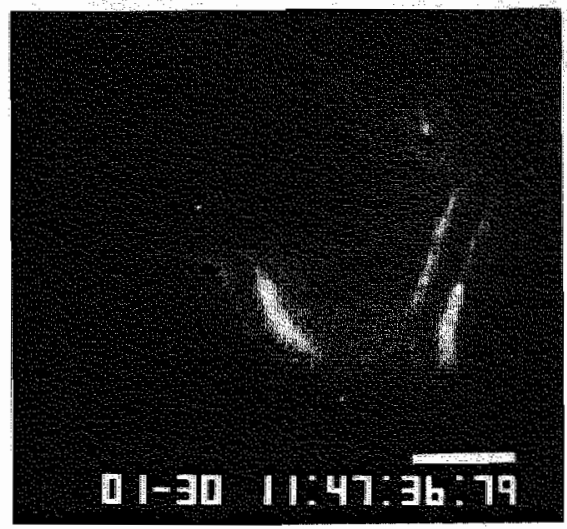

Fig. 7.2. Labeling of the wall of $a$ capillary in the soleus muscle of the rat with pyronin B. Fluorescent spots are nuclei. Parts of the capillary wall are out of focus. Bar, 20 $\mu m$.

Several dyes accumulated in or on the capillary wall. Of all the dyes tested, pyronin $B$ gave the best outline of the wall of microvessels in the mesentery. In addition, it yielded satisfactory visualization of the capillary wall in soleus muscle. The endothelial cytoplasm and nuclei, and possibly the endothelial glycocalyx and basal membranes, could be observed during several minutes after injection of the dye until the fluorescence faded to a low level. Unfortunately, injection of pyronin B caused a pronounced drop in arterial blood pressure (down to about 60\%) and red blood cell velocity (down to about $40 \%$ ) measured in mesenteric arterioles. Mean arterial blood pressure and mean red blood cell velocity increased gradually after the initial decrease and during the subsequent injections, but both were still below $75 \%$ of their initial values when background fluorescence became troublesome. This might limit its use in in vivo studies as long as these effects can not be counteracted. Mesenteric arteriolar diameter did not change as a result of the injections of pyronin $B$. If arteriolar diameters are indeed unaffected by pyronin $B$, than the decrease in red blood cell velocity was probably caused by a decrease in cardiac output.

A limited leakage of a fluorescent dye out of the circulation can be an advantage if the purpose is labeling of the vessel wall or blood cells, especially when the labeling is not very specific. When the blood plasma fluorescence decreases as a result of the leakage, structures which retain the dye may become more clearly visible. Based upon this mechanism, pyronin B allows visualization of the capillary wall, as has been shown in this study. A substance like acridin red can be successfully used to label thrombocytes (Tangelder et al., 1982). Acridine yellow can be used to visualize the nuclei of leukocytes in vivo and is now employed to differentiate leukocytes rolling in venules on the basis of their muclear shape (Janssens et al., 1989). However, dyes which leak out of the circulation rapidly and do not bind adequately to the structures of interest, can in general not be used in skeletal muscles because of the high background fluorescence. Acridin orange, that labels the microvascular wall in the mesentery (Tangelder et al., 1982), caused an unacceptably high background fluorescence in the soleus preparation. This was also the case 
with dyes such as neutralrot rein and acriflavin, which did accumulate in the capillary wall, but leaked rapidly out of the mesenteric circulation, thereby quickly increasing the background fluorescense. Therefore, in this study, these dyes were not tested in the soleus muscle preparation.

Some of the dyes tested are capable of labeling endothelial cells in vitro. In endothelial cell cultures, DiI-Ac-LDL is taken up by the cells and the fluorescent. probe Dil accumulates in lysosomal membranes (Voyta et al., 1984). In the present study, DiI-Ac-LDL did neither label the blood plasma nor did it accumulated in the capillary wall, indicating that the dye was rapidly removed from the circulation or was highly diluted in the blood plasma without accumulation in the endothelial cells. For labeling of cultured endothelial cells, a solution of $10 \mu \mathrm{g}$ Dil-Ac-LDL/ml medium is recommended (Voyta et al., 1984). Assuming that a rat of $250 \mathrm{~g}$ has a volume of blood plasma of about $10 \mathrm{ml}$ (Baker et al., 1980), then the $100 \mu \mathrm{g}$ that was injected should have been sufficient to label endothelial cells.

Lectins, such as Ulex europaeus Agglutinin (UEA) (Holthöfer et al., 1982; Ninomiya et al., 1985; Little et al., 1986), Concanavalin-A (ConA), Riccinus communis Agglutinin (RCA) and Wheat Germ Agglutinin (WGA) (Simionescu et al., 1982) have been used to label endothelial cytoplasm and/or its outer membrane in vitro, but none of these compounds accumulated at the endothelium to an extend high enough to contrast it from the background. Lectins are carbohydrate specific and might in part have been bound by sugar groups present in the circulation. It is conceivable that part of the lectins has in fact labeled the endothelial membrane in vivo (c.f. Simionescu et al., 1982), but that the labeling was masked by the fluorescence of the FITC conjugated lectins present in the blood plasma.

Labeling of the endothelial layer can be observed in tissue sections after perfusion of the tissue with thioflavin-S (Schlegel, 1949; Fourman and Moffat, 1967; Dohrmann and Wick, 1971). However, in our in vivo preparation the fluorescence of the dye in the vascular lumen probably hampered the visualization of the endothelial labeling.

A possiblity to label endothelium which was not tested in the present study is the use of antibodies against proteins of the luminal membrane of the cells, like antibodies against angiotensin converting enzyme (Ryan et al., 1976). By perfusion with such an antibody, the endothelial luminal membrane might be delineated in vivo. Some antibodies specific for endothelial cytoplasmic substances are known, e.g. anti factor VIII (Holthöfer et al., 1982; Little et al., 1986) and PAL-E (Schlingemann et $a l$, 1985). It is unlikely, however, that these will be usefull in vivo, because with an intact cellular membrane they will not reach their target.

In the mesenteric microvessels fluorescence of blood plasma could be observed for shorter or longer periods of time with most of the dyes tested. As expected, the large molecules conjugated with fluorescent dyes did not cause appreciable leakage. Lectins conjugated to FTTC labeled blood plasma similar to BSA-FITC which is conventionally used for in vivo visualisation of blood vessels, but appeared to extravasate even less. The lectins caused no visible alterations at the microcirculatory level and might be a good alternative for BSA-FITC as far as visualization of micro- 
vascular lumina is concerned. Some of the smaller dyes that leaked out of the circulation relatively slowly might have formed polymeres in wivo, like Thioflavis $S$ (Fourman and Moffat, 1967), or might have conjugated with plasma proteins, as is probably the case with evans blue (Kovács et al., 1975) and fluorescein-sodium (Moneta et al., 1987). The higher degree of leakage of fluorescein-sodium out of the circulation as compared to leakage of evans blue is in accordance with the finding that in vivo only $40 \%$ of fluorescein-sodium binds to plasma proteins (Brülisauer et al., 1986).

Some dyes tested in this study showed a strong photodynamic effect consisting of aggregation of thrombocytes upon excitation. Substances that induced thrombocyte aggregation and sticking in this way were acridin yellow, acriflavin, PLL-FITC, rose bengal, safranin $O$ and thioflavin-S. Most of these dyes labeled thrombocytes and/or endothelial cells and may have caused damage of these cells resulting in platelet aggregation. The respiratory problems observed after the administration of thioflavin-S might have been the result of emboli obstructing the circulation of the lung. Photodynamic effects of irradiated dyes have been reported by several authors and include temporal or persistent vasoconstriction, inhibition of capillary blood flow and thrombocyte aggregation (Algire and Schlegel, 1950; Rosenblum and ElSabban, 1977; Herrmann, 1983; Damon and Duling, 1984; Sato and Ohshima, 1984). Suggested causes of the photodynamic effects include heat accumulation (Kovács et al., 1975) and the formation of free radicals (Rosenblum and El-Sabban, 1977; Herrmann, 1983; Sato and Ohsima, 1984). Because of their strong photodynamic effect, some of the chemicals tested could be potential candidates for selective occlusion of vessels to create localized ischemic areas. This has been used in tumor treatment (Doiron and Gones, 1984).

In conclusion, capillary endothelium in skeletal muscle can be visualized with pyronin $\mathrm{B}$, although its use will be limited by the occurring drop in arterial blood pressure.

\subsection{SUMMARY}

To improve the optical definition of capillary endothelium in vivo, fluorochromes were tested in the rat mesentery and soleus muscle. Fluorochromes were injected intravenously. Endothelial cells, leukocytes and thrombocytes were visualized with the help of intravital fluorescence microscopy. Pyronin B labeled endothelial cells in the mesentery as well as in the soleus muscle preparation, yielding a clear outline of the capillary wall. Hoewever, injection of the dye caused a dramatic drop in arterial blood pressure and arteriolar red blood cell velocity. Neutralrot rein and acriflavin labeled endothelial cells in the mesentery. Because these dyes leaked out of the circulation rapidly, causing a high background fluorescence, they were not tested in the soleus preparation. Blood platelets were labeled by most ot the FITC conjugated lectins tested. Nuclei of endothelial cells and leukocytes were specifi- 
cally labeled by acridin yellow. Labeling of the blood plasma was observed with many dyes, but most of them leaked out of the circulation rapidly. On the other hand, especially the FITC conjugated lectins gave a stable, long term fluorescence of the blood plasma with minimal leakage. When excitated by light, some of the dyes tested caused a severe reduction or even cessation of capillary blood flow. In such cases, platelet aggregation was often observed. 


\section{MEASUREMENT OF SKELETAL MUSCLE CAPILLARY DIAMETERS IN VIVO}

\subsection{INTRODUCTION}

In vivo, objective and accurate determination of muscle capillary diameters is difficult. The contrast between the capillary wall and its environment is low. The dimensions of muscle capillaries are generally studied in thin muscles (Erikson and Myrhage, 1972; Myrhage and Hudlická, 1976; Damon and Duling, 1984; Dawson et al., 1987; Wiederhielm and Slaaf, 1987), but even in these preparations identification of the capillary wall is difficult and capillary segments with a well defined wall have to be selected. These limitations might introduce a certain bias. Labeling of the capillary lumen with fluorescent dyes can improve the visualization of capillaries (Mahler et al., 1983; Damon and Duling, 1984 and 1985; Brülisauer et al., 1986; Moneta et al., 1987). Because the core of the fluorescing capillary lumen is diffuse, Mahler et al. (1983) used intensity curves of video image lines of fluorescing capillary lumina to determine the capillary inner diameters objectively. Kaufman and Intaglietta (1985) used differentiated intensity lines of video recordings of trans-illuminated blood vessels to improve the accuracy to determine their diameter. In the present study, both techniques were combined in order to improve the determination of capillary inner diameters in muscle in vivo. Lumina of capillaries of rat soleus muscles were labeled with a fluorescent dye (FITC) conjugated to albumine and in this way could be studied down to a depth of about three muscle fibers. Capillary inner diameters were determined using differentiated signals of video recordings of the fluorescing capillary lumina. 


\subsection{MATERILS AND METHODS}

\section{Animals and tissue preparation}

Male Brown Norwegian or Wistar rats, $11-15$ weeks old and weighing $240-320 \mathrm{~g}$, were used. The animals were housed in a temperature controlled environment (23$26^{\circ} \mathrm{C}$ ) and fed with rat chow (type 1210, Hope Farms) and water ad libitum.

Animal and tissue preparation were similar to the ones discribed in chapter 7 . The rats were anesthetized with sodium-pentobarbital ( $60 \%$ solution (Narcovet); 1 $\mathrm{ml} / \mathrm{kg}$ body weight, i.p.). The animals were then tracheotomized and intubated to facilitate breathing. Anesthesia was maintained through a continuous infusion of sodium pentobarbital $(7.5 \mu / \mathrm{kg} / \mathrm{min})$. The right femoral vein was cannulated for injection of BSA-FITC.

\section{Soleus muscle preparation:}

The animal was placed in a prostrate position. The foot of the left hind limb was fixed to minimize movement. The dorsal side of the skin of the lower hind limb was incised. The gastrocnemius muscle, overlying the soleus muscle, was carefully split, causing as little trauma as possible. The two halves were pulled aside with suture material (Ethibond 6-0) until the soleus muscle became accessible. The connective tissue overlying the soleus was carefully removed. Subsequently, the animal was placed under the microscope. The tissue was kept moist by covering it with wet gauze, except for the part under observation which was continuously superfused with Tyrode solution gassed with $95 \% \mathrm{~N}_{2} / 5 \% \mathrm{CO}_{2}\left(37^{\circ} \mathrm{C}\right.$; $\left.\mathrm{pH} 7.4\right)$. Only undamaged vessels that did not show compromized blood flow were used for analysis.

\section{Mesentery preparation:}

Because the wall of small microvessels can be observed directly in the mesentery, this tissue was used to compare epi-illuminated images of the microvessels used for fluorescence with trans-illuminated images. Through a lateral incision in the abdomen the coecum and distal ileum were brought outside. Care was taken to minimize tissue trauma. The second module of the mesentery, proximal of the coecum, was spread over a siliconized glass plate mounted in an electrically heated microscope table $\left(37^{\circ} \mathrm{C}\right)$ and the intestine was kept moist with overlying wet gauze. The preparation was continuously superfused with buffered Tyrode solution (see above). Whenever damaged vessels were observed, the next module of the mesentery was prepared for observation. Only vessels with no excessive number of sticking or rolling leukocytes in the venueles were used for analysis. 
Microscope and recording system

Microscope and recording system were similar to the ones discribed in chapter 7. All observations were made with a Leitz intravital microscope adapted to tele scopic imaging (Slaafet al., 1982). For trans-illumination of the mesentery preparation, bright field microscopy using a tungsten lamp was used. Incident illumination of the soleus muscle was performed using a Xenon (150W) arch. Fluorescence microscopy was performed with an incident fluorescence illuminator (Leitiz Ploemopak 2.2; tube factor 1.6), using ax50 water-immersion objective ( $\mathrm{SW} 50$; numerical aperture 1.00) and interchangeable filter set (Leitz I2; BP 450-490, RKP 510 , LP 515). The tissues were observed directly with a 10 ocullar. Images were recorded using a video camera (SIT, Bosch) and a sensitive video recorder (Sony, Betamax). Final magnification at the front plane of the TV camera was $\times 100$. Under the experimental conditions, the resolution of the optical and of the recording sys-

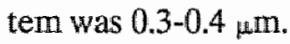

\section{Determination of capillary diameter}

To visualize the capillary lumen in mesentery and soleus muscle preparations, $30-50 \mathrm{mg}$ bovine serum albumin (BSA) labeled with fluorescein-isothiocyanate (FITC) was injected intravenously. To reduce photodynamic effects (Damon and Duling, 1984; Sato and Ohshima, 1984; Dawson et al., 1987; Reed and Miller, 1988), the TV camera was used at a high sensitivity setting, the illumination intensity was reduced by means of a $30 \%$ gray filter and the observation time was limited (generally less than $15 \mathrm{~min}$ ). The microscope was focused on the widest part of the capillaries and capillary images were positioned about perpendicular to the monitor screen lines, using a K-prism (Figures 8.1A and 8.2A).

After the actual experiment, selected images of the video recording were stored in a frame buffer of a personal computer (IBM). The intensity of each pixel could range from 0 (black) to 255 (white). Pixel width and height of the capillary image corresponded in the preparation to 0.26 and $0.17 \mu \mathrm{m}$, respectively. An intensity curve of the fluorescing capillary lumen was produced by the image processor by averaging 10 subsequent (horizontal) image lines, corresponding with a vessel segment length of about $1.7 \mu \mathrm{m}$. In this way the electronic noise was reduced to acceptable levels (compare Figures $8.2 \mathrm{C}$ and $8.2 \mathrm{D}$ ). An additional curve, representing the deriwative of the averaged intensity curve was generated by the computer (Figures $8.1 \mathrm{C}$ and $8.2 \mathrm{D}$ ). The horizontal distance between the maximal and the minimal value of the differentiated intensity line, corresponding with the position of the two steepest parts of the original intensity line, was determined by vertical displacement of a cursor and used to calculate capillary diameters. A calibration grid with an in-m terline distance of $10 \mu \mathrm{m}$ was used for calibration. By combining fluorescence and trans-illumination, in the mesentery preparation the diameter calculated from the 

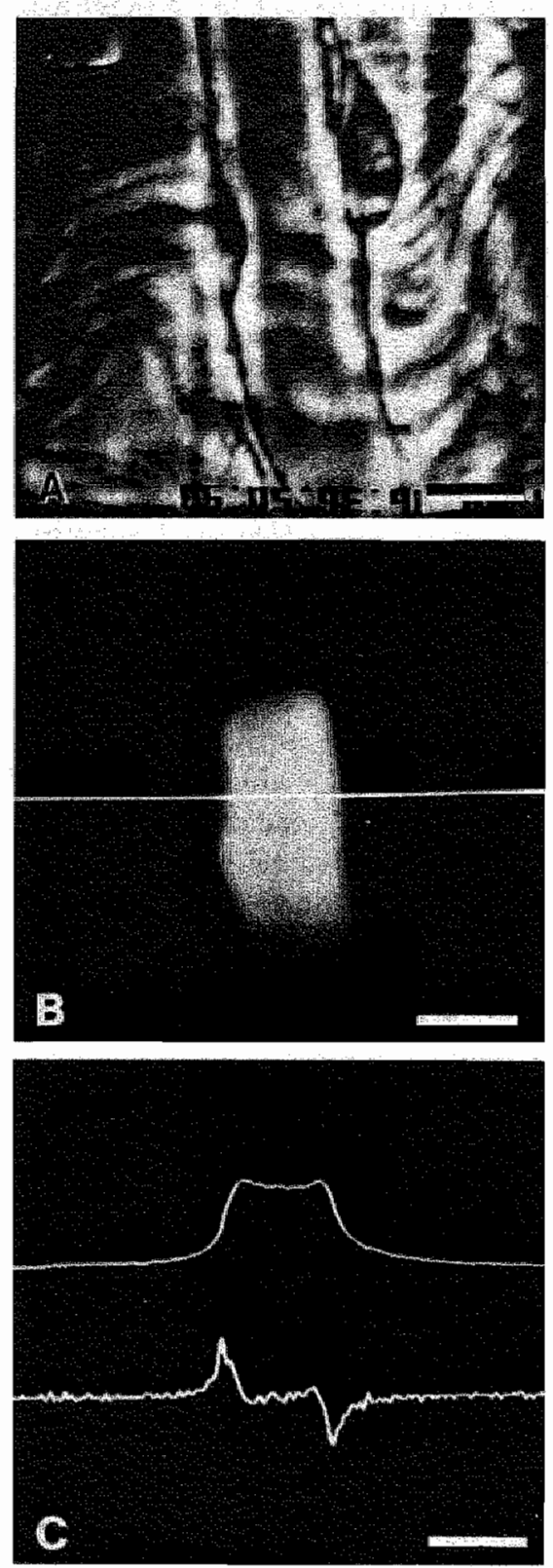

differentiated intensity line was compared with the vessel diameter observed in the trans-illuminated image.

\subsection{RESULTS}

About $15 \mathrm{~s}$ after injection of BSAFITC, the lumen of the capillaries of the soleus muscle became fluorescent. BSAFITC showed little leakage out of the circulation and no leaky spots were observed. A clear outline of the fluorescent capillary lumina could be obtained down to a depth of about three layers of muscle fibers. The median inner diameter of rat soleus muscle capillaries, as calculated from the differentiated mean intensity curve of a small capillary segment, was $5.7 \mu \mathrm{m}$ (58 capillaries, 5 rats). The diameters ranged from 4.3 to $7.9 \mu \mathrm{m}$. The frequency distribution of the capillary inner diameters is shown in Figure 8.3. The distribution is slightly

Fig. 8.1. Determination of inner diameter of mesenteric microvessel of rat using image analysis. (A) Microvessel of mesentery. (B) Fluorescent lumen of the same microvessel (small diaphragm used). (C) Intensity curve of the average of 10 subsequent $T V$ lines, starting with the one indicated in B (upper line) and the derivative of this intensity curve (lower line). Note that two clear peaks can be dicriminated in the differentiated line that correspond to the steepest parts of the upper curve. The (horizontal) distance between the peaks corresponds with the inner diameter of the microvessel. Bars represents $10 \mathrm{um}$. 

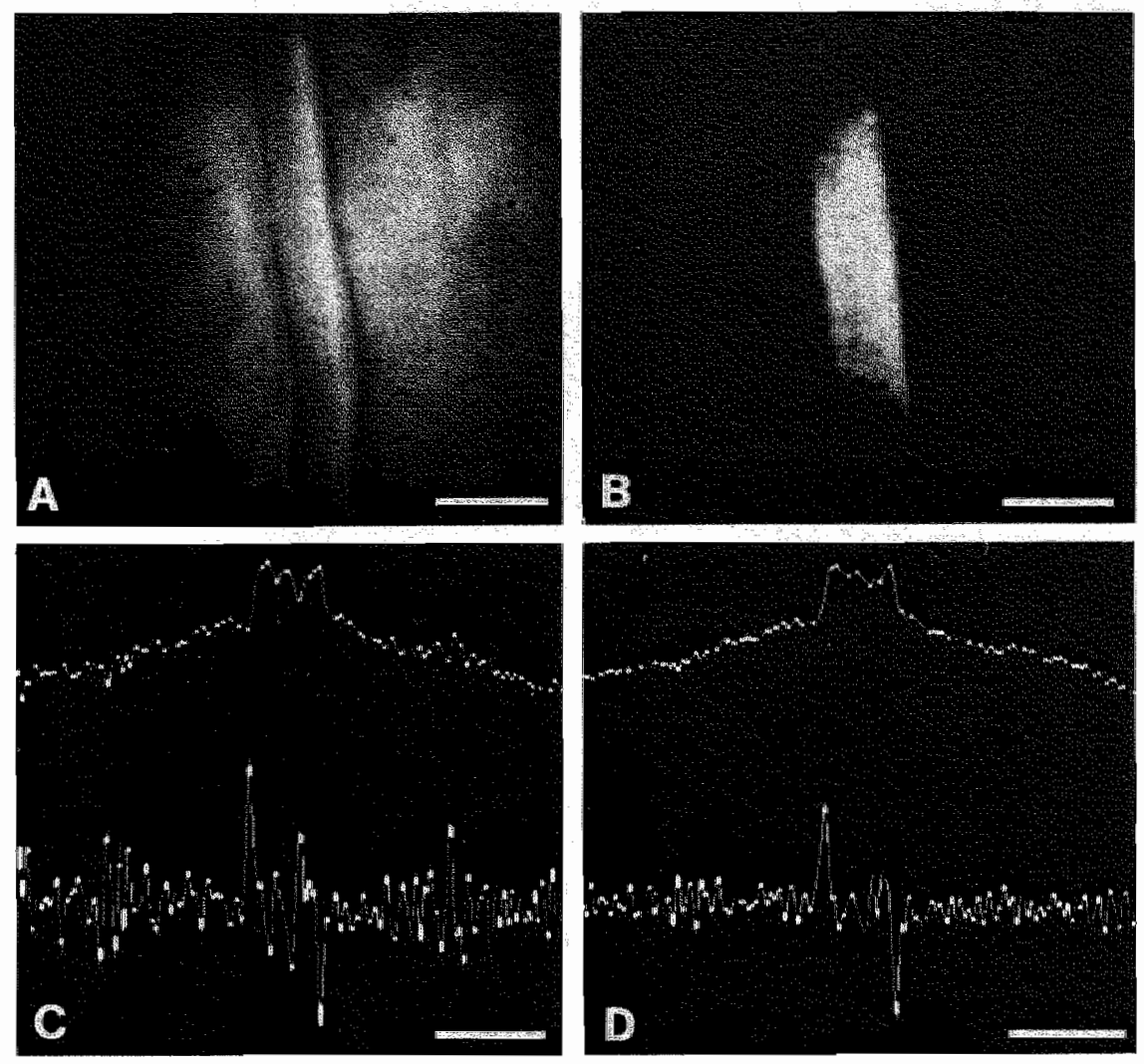

Fig. 8.2. Determination of capillary inner diameter of rat soleus muscle. (A) Capillary wisualized by side-illumination. This kind of visualization could only be achieved with part of the capillaries. (B) Fluorescent lumen of the same capillary (small diaphragm used). (C) and $(D)$ : Intensity curve of $I(C)$ or of the awer age of 10 subsequent $T V$ lines (D) from the center of $B$ (upper lines), and the derivatives of these curves (lower lines). Note that that the signal to notse ratio is reduced by averaging of the lines. Bars represent $10 \mu \mathrm{m}$.

skewed with its longest tail towards the larger values. The coefficient of variation (standard deviation divided by the mean) of the inner diameter masurements in a single capillary section (soleus muscle preparation) that was defocussed and focussed before each measurement, was 0.03 (inner diameter $7.2 \mu \mathrm{m} ; \mathrm{n}=10$ ). The largest deviation from the mean value, as obtained with repeated measurements in the same capillary, was 7\%, indicating that the method used can determine differences between capillary inner diameters accurately.

Because the wall of the microvessels in the mesentery can be observed directly by means of trans-illumination, the inner diameter of a microvessels as assessed with 


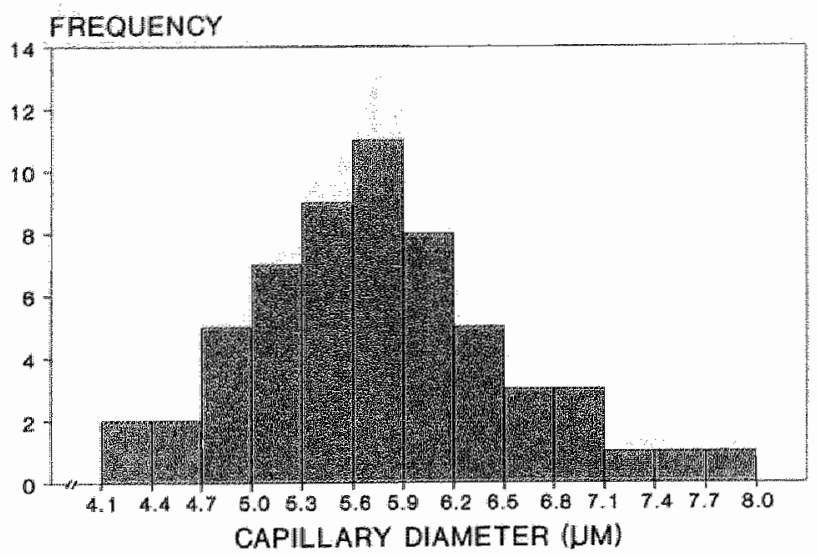

Fig. 8.3. Frequency distribution of capillary inner diameters of rat soleus muscle, as determined in wivo.

this technique could be compared with the inner diameter as calculated from the fluorescing lumen with epi-illumination. The inner diameter of the microvessels in the mesentery corresponded well with the (horizontal) difference between the maximal and minimal values of the integrated intensity line, as is illustrated in Figure 8.1. Similar results were obtained from the soleus muscle preparation, using capillary segments that could be visualized with side-illumination (Figure 8.2). Under the experimental circumstances, the calculated capillary inner diameter in the soleus muscle was independent of the amount of bovine serum albumin-FITC injected (20 $\mathrm{mg}$ vs $40 \mathrm{mg}$ ), blood flow velocity (normal flow vs no flow) and the electronic amplification of the image $(P<0.05 ; n=10$; Mann-Whitney $U$ test $)$. In addition, peak values of the differentiated intensity curve were maximal when the image was in focus according to the experimentator and calculated capillary dimensions remained within $5 \%$ of their initial value if the image was $1 \mu \mathrm{m}$ out of focus.

\subsection{DISCUSSION}

To determine capillary diameters in rat soleus muscle in vivo, fluorescent labeJing of plasma with FITC-BSA was combined with image analysis. Averaging of ten image lines (about $1.7 \mu \mathrm{m}$ vessel length) of horizontally oriented capillaries, yielded an intensity curve with little background noise. In the differentiated mean intensity line, the steepest parts of this line could clearly be discriminated. The distance between the steepest parts of the intensity line corresponded well with the inner capillary diameter, as detemined in the rat mesentery by trans-illumination and the 
soleus muscle by side-illumination. Capillary diameters thus determined were independent of the amount of BSA-FITC present in the lumen and the electronic magnification of the image. The advantages of the technique used in this study are that 1) the fluorescent lumen of virtually all capillaries down to a depth of about three muscle fibers can be clearly visualized, 2) the use of image analysis is objective and 3) capillary inner diameters can be determined accurately.

In the soleus muscle the capillary inner diameters varied between 4.3 and $7.9 \mu \mathrm{m}$ (median value $5.7 \mu \mathrm{m}$ ). These data are in the same range as the capillary inner diameters measured in vivo in other striated muscle preparations of adult rats and cats (mean values 4.0-5.6 $\mu \mathrm{m}$; Erikson and Myrhage, 1972; Myrhage and Hudlicka, 1976; Dawson et al., 1987; Wiederhielm and Slaaf,1987) or in rat corrosion casts (4.8-5.0 $\mu \mathrm{m} ;<1 \%$ shrinkage; Potter and Groom, 1982). On the other hand, mean capillary inner diameters, as determined in chemically fixed and plastic embedded muscles, appear to be much smaller. In the soleus muscle of rats of similar age and weight, the mean capillary inner diameter in plastic sections is about $4.2 \mu \mathrm{m}$ (chapter 3). The mean values as obtained in plastic embedded tissue of other striated muscles, varies between 2.7 and $4.2 \mu \mathrm{m}$ (Jerusalem et al., 1.974A; Brodal et al., 1977; Mäkitie, 1977; Gidlöf et al., 1987A and 1987B). The smaller soleus capillary diameters in plastic sections ( $4.2 \mu \mathrm{m}$; chapter 3 ), as compared with the in wivo diameter of $5.7 \mu \mathrm{m}$ (this chapter) indicates the occurence of a lateral tissue shrinkage, a generally accepted phenomenon (Glauert, 1975), of 26\%. Shrinkage may occur during fixation, dehydration and/or embedding in plastic but is assumed to be limited, e.g. about 5\% linear shrinkage, especially when Epon is used for embedding (Glauert, 1975; Aherne and Dunnill, 1982). Brodal et al. (1977) found a linear shrinkage of $28 \%$ of muscle fibers, but they compared muscle fixed by immersion with cryofixed muscle without correction for differences in sarcomere lengths. In addition, their tissue was embedded in a different resin (TAAB), which may cause a larger tissue shrinkage. One could argue that part of the discrepancy between the capillary diameters, as assessed in vitro and in vivo, results from the selection of large, well perfused capillaries in the in vivo preparation due to the fact that the capillary wall is often difficult to identify (Damon and Duling, 1984; Dawson et al., 1987). This, however, is unlikely because in the present study the results obtained with fluorescent labeling of the capillary lumen were not different from those obtained in the studies without fluorescence. Because the superfusate lacks colloids, it could be argued that fluid accumulates in the capillary lumen due to osmotic effects. To achieve an increase in capillary diameter of about $30 \%$, such a fluid accumulation must have caused a local increase in capillary transmural pressure of at least $20 \mathrm{~mm} \mathrm{Hg}$ (Bouskela and Wiederhielm, 1979; Davis, 1988). This is unlikely because capillaries are open structures.

It cannot be excluded that capillaries near the surface of the muscle, that are the ones examined in the present study, might be larger than the deeper lying capillaries, which are generally examined in sections. Therefore, at the present state of art it is uncertain whether the discrepancy between the capillary inner diameters, 
as assessed in vivo and in plastic sections, can solely be attributed to tissue shrinkage alone.

In conclusion, the combination of fluorescent labeling of the vascular lumen and image analysis, as used in this study, is an objective and accurate method to determine inner capillary diameters in skeletal muscle in vivo.

\subsection{SUMMARY}

Fluorescent labeling of the capillary lumen with bovine serum albumin (BSA) conjugated with fluorescein-isothiocyanate (FITC) was used to determine capillary diameters of the rat soleus muscle in vivo. Video recordings of fluorescing capillary lumina, oriented perpendicular to the (horizontal) lines of the TV-screen, were used for image analysis. Intensity curves of ten subsequent (horizontal) image lines were averaged. The derivative of the averaged intensity curve was used to determine capillary inner diameters. The method used was objective and accurate. In mesentery preparations of the rat it was demonstrated that the calculated capillary inner diameter corresponded to the true inner diameter. The method was independent of the amount of BSA-FITC present in the lumen, blood flow velocity and the electronic amplification of the image. The median inner capillary diameter of the soleus muscle was $5.7 \mu \mathrm{m}$ (range 4.3 to $7.9 \mu \mathrm{m} ; \mathrm{n}=58 ; 5$ rats), being about one third larger than the capillary inner diameters in plastic cross-sections of the rat soleus muscle, as referred to in the literature. 


\section{GENERAL DISCUSSION}

In rats unaccustomed exercise may lead to muscle damage. In the involved muscles, usually a small part of the fiber population is affected. The fiber damage is characterized by focal degeneration and invasion by inflammatory cells. The damage is maximal 24-48 hours after the exercise and is usually followed by complete muscle fiber repair about one week later (Kuipers et al., 1983).

Such factors as ischemia and mechanical rupture of the myofibers may be involved in the exercise induced degenerative process. It was the main objective of the present study to investigate in the rat whether and to which extent ischemia contributes to the muscle fiber degeneration. Ischemia might be involved in the exercise induced degenerative process because microscopically the degeneration and invasion of muscle fibers is similar to that observed following ischemia. In addition, the finding that the length of the degenerated segments of affected fibers is similar to the length of the capillaries that supply them (see chapter 2) indicates the existence of impaired perfusion and, hence, local ischemia. In the present thesis degeneration of rat soleus muscle fibers as a result of a bout of uphill running was used as a model.

The results of the studies described in this thesis do nót support the hypothesis that ischemia is the primary cause of exercise induced muscle fiber degeneration, In the time interval between the exercise and the observed muscle fiber degeneration, the vascular bed of the soleus muscle was unobstructed and capillary morphology as well as intramuscular pressure were normal. Although it was not possible to study capillaries adjacent to muscle fibers before degeneration, because these fibers can not be discriminated at an early stage, no obvious local capillary alterations or obstructions were observed in any of the sections at the light or electron microscopic level immediately following exercise. However, because the existence of ischemia, if any, was not measured directly a possible involvernent in the process of exercise induced muscle fiber degeneration can as yet not be excluded completely.

Light- and electron-microscopic observation of sections of the soleus muscle of exercised and non-exercised rats showed that perfusion with the fixative had removed all blood cells from capillaries and other blood vessels. Clusters of fixed 
plasma proteins in the blood vessels, that are usually observed in ultrathin sections of tissue fixed by immersion, were not found. This indicates that plugging of blood vessels by blood cells was absent and that all blood vessels had been flushed. No obstruction of the capillary lumen by intraluminal protrusion of endothelial nuclei was observed. The dimensions of the capillary lumina were normal and no post-exercise swelling or other changes of the endothelial cells could be found. Degenerated muscle fibers were supplied with a normal amount of capillaries. The finding that these capillaries were dilated and larger than other capillaries indicates a preferential shunting of blood and blood cells towards the affected area at this time (Blixt et al., 1987; Schmid-Schönbein et al., 1980), rather than ischemia. The higher vulnerability to uphill running of type I fibers, as compared to type II fibers of rat soleus muscle (Kuipers at al., 1983), could not be related to differences in density or dimensions of their supplying capillaries. In addition, the finding in this study that muscle fibers adjacent to degenerated fiber segments appeared to be morphologically unaffected indicates that muscle fiber degeneration is restricted to individual fibers rather than to a small area around individual capillaries or a group of capillaries (chapters 3 and 4 ).

In addition to the possibility of vascular obstructions, another factor that may compromize muscle perfusion is exercise induced swelling of the muscle resulting in an increase in tissue pressure. Prior to the degeneration of individual muscle fibers 24 hours after running, muscle fibers as well as the soleus muscle as a whole swelled considerably, reaching a maximum at 6 hours after running (20-30\% increase in volume). However, swelling of the muscle did not result in an increase in intramuscular pressure which remained at control levels (about $1 \mathrm{~mm} \mathrm{Hg}$ ) between 18 minutes and 24 hours after running. This indicates that the running induced muscle swelling does not cause an increase in intramuscular pressure to a level high enough to cause ischemia and subsequent muscle fiber damage (chapter 5). The fiber swelling, as determined in plastic sections, was associated witln an increase in spacing of the thick contractile filaments from $28.4 \pm 1.4 \mathrm{~nm}$ in control animals up to $30.7 \pm 2.1 \mathrm{~nm}$ at 6 hours after exercise (mean $\pm \mathrm{SD} ; \mathrm{n}=15$ fibers; 3 rats per group; $P<0.01$; unpublished result), indicating a swelling of the filament lattice by $17 \%$, which is close to the value observed for the fiber swelling. Because muscle fiber swelling and filament spacing are related to a reduction in contraction speed and tension development (Metzger and Moss, 1987), these changes might, at least partly, explain the temporaly impaired muscle function that is often observed after exercise.

In chapter 4 a newly developed retrieval method is described, in which fibers were classified in plastic sections using a retrieval method. Slow twitch fibers were found to stain darker with toluidine blue than fast twitch fibers. In the soleus muscle, gastrocnemius muscle and peroneus muscle, the staining intensity of the muscle fiber cross-sections, as measured by the amount of light absorption, was never lower in type I than in type Ill fibers and was inversely related to the spacing of the thick contractile filaments. In all three muscles studied, the spacing of the thick filaments was smaller (mean 7\%) in slow twitch than in fast twitch fibers (chapter 6). Because 
the myosin heavy chains of fast and slow twitch muscle fibers have different biochemical properties (Lowey and Risby, 1971; Whalen et al., 1979; Staron and Pette, 1986; Eddinger and Moss, 1987), it is conceivable that differences in the molecular architecture of the thick filaments, e.g. their thickness or the length of their heads that can form the cross-bridges, explain the difference in spacing.

Filament spacing is helpful in muscle fiber typing in plastic embedded cross-sections without the use of histochemical or immunocytochemical techniques In $92 \%$ of adjacent pairs of slow twitch and fast twitch fibers in the soleus, gastrocnemius and peroneus muscles (combined data) the spacing was larger in fast twitch than in slow twitch fibers. Although some overlap exists, at present filament spacing, and to a more limited extent staining intensity, appears to be the only method to directly discriminate between slow twitch and fast twitch fibers in Epon cross-sections. Fiber types can be discriminated on the basis of morphological differences of Mline and Z disc (Salmons et al., 1978; Sjöström et al., 1982; Thornell et al., 1987), but this can only be done in longitudinal sections and the classification by means of these structures is not always conclusive either.

Because microscopic techniques may yield artifacts and can, by definition, only give a momentary impression of the state of the tissue, in the last two experimental chapters (7 and 8) an attempt was made to study capillary morphology directly in vivo. In chapter 7, we searched for fluorescent dyes that accumulate in or on endothelial cells in order to improve the visualization of capillaries in vivo. Of all dyes tested, only pyronin B labeled the capillary endothelium, and possibly the endothelial glycocalyx and basal membranes, to an acceptable level in the mesentery as well as in the soleus muscle. However, the labeling of the capillary wall by pyronin $B$ was temporarily, while injection of the dye caused a considerable drop in systemic arterial blood pressure and blood flow in the mesenteric microvessels. These side effects might limit the use of pyronin B for in viwo studies of capillaries in skeletal muscle.

In the last study (chapter 8), we determined the inner diameter of capillaries of the rat soleus muscle in wivo. The dimensions of rat soleus muscle capillaries appear to be larger in vivo (average diameter about $5.7 \mu \mathrm{m}$ ) than in plastic sections (average diameter about $4.2 \mu \mathrm{m}$ ) (c.f. chapter 3,4 and 8). This finding is in agreement with data from the literature obtained in other muscles. In these studies, the mean capillary diameter was also found to be larger in in wivo preparations (4.0-5.5 $\mu \mathrm{m}$; Erikson and Myrhage, 1972; Myrhage and Hudlicka, 1976; Dawson et al, 1987) than in plastic sections (2.7-4.2 $\mu$ m; Jerusalem et al., 1974B; Brodal et al., 1977; Makitie, 1977; Gidlöf et al., 1987A and 1987B; ). Although it is generally accepted that tissue shrinkage occurs during the embedding process (Glauert, 1975; Aherne and Dunnill, 1982), a tissue shrinkage of $26 \%$ (chapter 8 ) is tather high. This discrepancy needs further inwestigation. Since in the in wwo preparation only superficial capillaries are examined, a selection of larger capillaries in the vivo situation can not be excluded.

Tissue shrinkage might have influenced the absolute walues of our morphometric data in all studies in which tissue sections were morphometrically analyzed 
(chapters 3,4 and 6). However, by comparing the data to control values obtained from tissues processed in the same way, the influence of shrinkage is likely to be eliminated.

As an alternative to muscle fiber degeneration caused by ischemia, it might be hypothesized that mechanical rupture initiates the degenerative process. None of the results from the present thesis contradict the hypothesis of mechanical rupture, although the results do not support it directly either. The soleus muscle is adapted to maintain body bearing and is in untrained animals probably not accustomed to the high peak loads that occur during running. During uphill running the muscle has to shorten extensively and higher peak forces are developed than at rest. Under this condition, as well as during downhill running, the soleus muscle might be especially susceptible to rupture of muscle fibers. The hypothesis of mechanical rupture is supported by some findings in the literature. It has been shown that membrane rupture induced by forced lengthening during tetanic contraction of muscles can cause focal fiber degeneration and invasion by inflammatory cells, which are very similar in time course and appearence to the ones caused by exercise (McCully and Faulkner, 1986; Stauber et al., 1988). Disruption of sarcomeres in myofibrils after exercise has been reported (Fridén et al., 1981 and 1983; Fridén, 1984). Armstrong and colleagues (1983) argue that eccentric exercise is far more effective in causing muscle fiber damage than concentric exercise, because the tension produced during eccentric contraction is probably distributed over a smaller number of active fibers and the resulting higher forces per active cross-sectional fiber area might increase the amount of fiber disruption.

In conclusion, unaccustomed exercise may lead to loss of integrity of individual muscle fibers. The present data suggest that, although not investigated directly, ischemia is not likely to be the primary cause of the exercise induced focal muscle fiber degeneration. 


\section{REFERENCES}

Addicks, K., H. Wejgelt, G. Hanck, D.W. Lübbers and H. Knoche. Light- and electronmicroscopic studies with regard to the role of intraendothelial structures under normal and inflammatory conditions. Microcirculation in Inflammation. Bibl. Anat. 17:21-35. 1979.

Aherne, W.A. and M.S. Dunnill. Morphometry, Edward Arnold Ltd. London. 1982.

Algire, G.H., and J.U. Schlegel. Circulatory reactions in photodynamic action. J. Cell, Comp. Physiol. 35:95-110. 1950.

Andersen, P. Capillary density in skeletal muscle of man. Acta Physiol. Scand. 95:203-205. 1975.

Andersen, $P_{*}$ and A.J. Kroese. Capillary supply in the soleus and gastrocnenius muscles of man. Plitgers Arch. 375:245-249. 1978.

Armstrong, R.B., R.W. Ogilvie and J.A. Schwane. Eccentric exercise-induced injury to rat skeletal muscle. J. Appl. Physiol. 54(1):80-93. 1983.

Ashton, H. The effect of increased tissue pressure on blood flow. Clin. Orthop. 113:15-26. 1975.

Asmussen, E. Observations on experimental muscular soreness. Acta Rheum. Scand 2: 109-116. 1956.

Bagge, U, and M. Braide. Leukocyte plugging of capillaries in wivo. In: White Blood Cells. Eds.: Bagge U., Born G.V.R. and Gaehtgens P. Martinus Nijhoff Publishers, The Hague, The Netherlands. 8998. 1982 .

Baker H.J, J.R. Lindsey and S.H. Weisbroth. The Laboratory Rat. Volume II. Research Applications. Academic Press, New York. 1980.

Bartsch, R.C., E.E. McConnell, G.D. Imes and J.M. Schmidt. A revieuw of exertional rhabdomyolysis in wild and domestic animals and man. Vet. Pathol. 14:314-324. 1977.

Beeston, B.E.P., R.W. Horne and R. Markham. Electron diffraction and optical diffraction techniques. Chapter 4: Analysis of the electron diffraction pattern. In: Practical methods in clectron mycroscopy. Eds.: Glauert A.M. American Elzevier Publishing Co., New York. 223-251. 1972.

Bennett, H.S., J.H. Luft and J.C. Hampton. Morphological classification of vertebrate blood capillaries. Am. J. Physiol. 196(2):381-390. 1959.

Blixt, A., M. Braide, R. Myrhage and U. Bagge. Vital Microscopic studies on the capillary distribution of leukocytes in the rat cremaster muscle. Int. J. Microcirc: Clin. Exp. 6:273-286. 1987.

Bodine $S_{s} . E_{t}$ R.R. Roy, E. Eldred and V.R. Edgerton Maximal force as a lunction of anatomical features of motor units in the cat tibialis anterior. J. Netrophysiol. 56:1730-1745. 1987.

Bouskela, $\mathrm{E}_{\text {, }}$ and C. Wiederhielm. Viscoelastic properties of capillaries. Microvasc. Res. 17(3):S1,1.2. 1979.

Brodal, P., F. Ingjer and L. Hermansen. Capillary supply of skelletal muscle fibers in untrained and endurance-trained man, Am. J. Physiol. 232(6):H705-H712. 1977.

Brooke, M.H., and K.K. Kaiser. Muscle fiber types: how many and what kind ? Arch. Meurol. 23:369 379. 1970 . 
Writisaver, M., M. Moneta and A. Bollinger. Indocyanine green and Na-fluorescein used in a double tracer technique for the study of buman skin microcirculation. In: Joint meeting 1986, Heidelberg/FR. British microcirculation society and Gesellschaft fut Mikrozirkulation. Deutsche Abbot GmbH, Wiesthaden/FR G. 171-172. 1986.

Byrnes, W.C, P.M. Clarkson, J.S. White; S.S. Hsieh, P.N. Frykman and R J Maughan. Delayed onset ming the soreness following repeated bouts of downhill running. J. Appl. Physiol. 59(3):710-715. 1985.

Cabric, M., H.-J. Appll and A. Resic. Steriological analysis of capillaries in electrostimulated human muscles. Int. I. Sports Med. 8327-330. 1987.

Casley-Smith, J.R. Freeze-substitution of capillary endothelum: The artefactual nature of trans-endothelial channels and the forms of attached vesicles. Micron 11:461-462. 1980 .

Casley-with, J.R. Freeze-substitution observations on the endothelium and the passage of ions. Microcirculation $1(1): 79,109,1981$.

Cotter, $M, O$. Hudlickd and $G_{*}$ Vrbova. Growth of capillaries during long-term activity in skeletal muscle. Bibl. Anat. 11:395-398. 1973.

Damom, D.H, and B.R. Duling. Distribution of capillary blood flow in the microcirculation of the hamister: an in vivo study using epifluorescent microscopy. Microvase. Res. 27:81-95. 1984.

Damon, $\mathrm{D}, \mathrm{H}$, and $\mathrm{B} . \mathrm{R}$. Duling. Evidence that capillary perfusion heterogeneity is not controlled in striated muscle. Am. J. Physiol. 249,H386-H392. 1985.

Davis M.J Microvascular control of capillary pressure during increases in local arterial and venous pressure. Am. J. Physiol 254:H772-H784, 1988.

Dawson, J.M., R.K. Tyler and O. Hudlicka. A comparison of the microcirculation in rat fast glycolytic and slow oxidative muscles at rest and during contractions. Microwasc. Res. 33:167-182. 1987.

Desjardin C. and B.R. Duling. Enzymatic modification of red cell distribution within capillaries (Abstract). Int. J. Microcirc.: Clin and Exp. Special issue. 111.1988.

DiBona, D.R., and W.J. Powell, Jr. Quantitative correliation between cell swelling and necrosis in myocardial ischemia in dogs. Circ. Res. 47:653-665. 1980.

Dodscon, A., J. Garson, M. Burke, B.H and Anderton. Monoclonal antibody that detects human type I muscle fibers in routinely fixed wax embedded sections. J. Clin. Pathol. 40.172-174. 1987.

Dohrmann, G.J., and K.M. Wick. Demonstration of the microvasculature of the spinal chord by intrawenous injection of the fluorescent dye, Thioflavine S. In: Stain Technology. The Williams \& Wilkins Company: Baltimore 46:321-322. 1971.

Doiron, D.R. and C.J. Gones (Eds.). Porplyrin localization and treatment of tumors. A.R. Liss. New York. 1984.

Dubowitz, V. Muscle Biopsy. 2nd Edition. Hailliere Tindall. 37-38. 1985.

Eddinger T.J. and R.L. Moss. Mechanical properties of skinned single tibers of identified types from rat diaphragm. Am. J. Physiol. 253:C.210 C218. 1987.

Eriksson, E, and R. Myrhage. Mïrovescular dimensions and blood flow in skeletal muscle. Acta Physiol. Scand. 86:211-222.1972.

Fontrman,, , and D.B. Moffat. Fluorescence techniques in the study of the microcirculation of the kidney, Im: in wo techniques in histology. Ed. G.H. Bouine. The Whlliams \& Wilkins Company. Baltimore $239-259,1967$.

Frickn, J., M. Sjöström and B. Ekblom. A morphological study of delayed muscle soreness. Experiuntia $37: 506-507$. 1981 .

Fridlen, $J_{,}$M. Sjöströom and B. Ekblom. Myofibrillar damage following intense cecentric exercise in man. Int. J. Sports Med. 4:170-176. 1983.

Friden, J. Muscle soremess after exercise: implications of morphological changes. Int. J. Sports Med. $5: 57-66.1984$.

Friden, J., P.N. Sfakianos and A.R. Hargens. Muscle soreness and intramuscular Tuid pressure: comparison between eccentric and concentric load. J. Appl. Physiol. 61(6):2175-2179. 1986.

Friden, $J_{,}$, P.N. Sfakianos, A.R. Hargens and W.H. Akeson. Residual muscular swelling anter repetitive eccentric contractions. J. Orth. Res, 6:493-498. 1988. 
Garnett, R.A.F. M.J. O'Donovan, J.A. Stephens and A. Taylor. Motor unit organization of human medial gastrocnemius. J. Physiol. 287:33-43, 1978.

Geller, S.A. Extreme exertion rhabdomyolysis. Hum. Pathol, 4(2):241-250. 1973.

Gidlöf, A., D.H. Lewis and F. Hanmersen. Fine structure of the human skeletal muscle capillary. A morphometric analysis. Int. J. Microcirc: Clin. Exp. 7:43-66. 1987 A.

Gidlöf, A, D.H. Lewis and F. Hammersen. The effect of prolonged total isehemia on the ultrastructure of human skeletal muscle capillaries. A morphometric analysis. Int. Jl. Microcirc: Clin. Exp. 7:67-86. $1987 \mathrm{~B}$.

Glauert, A.M. Fixation, dehydration and embedding of biological specimens. North-Holland Publishing Company, Amsterdam, Oxford. 1-4. 1975.

Gollnick, P.D., and C.D. Ianuzzo. Colonic temperature response of rats duringexercise. J. Appl. Physiol. $24(6): 747-750.1968$.

Gonzalez-Serratos, H. A.V. Somlyo, G. McClellan, H. Shuman, L.M. Borrero and A. Somlyo. Composition of vacuoles and sarcoplasmic reticulum in fatigued muscle: Electron probe analysis. Proc. Natl. Acad. Sci. 75(3):1329-1333. 1978.

Gray, S.D., and E.M. Renkin. Microvascular supply in relation to fiber metabolic type in mixed skeletal muscle of rabbits. Microvasc. Res. 16:406-425. 1978.

Hamilton, R.W., L.B. Gardner, A.S. Penn and M. Goldberg. Acute tubular necrosis caused by exe rcise-induced myogllobinuria. Amn. Intern. Med. 77:77-82, 1972.

Hargens, A.R., S.J. Mubarak, C.A. Owen, L.P. Garetto and W.H. Akeson. Interstitial fluid pressure in muscle and compartment syndromes in man. Microvasc. Res. 14:1-10. 1977.

Hasselgrove, $J, C$, and H.E. Huxley. X-ray evidence for radial cross-bridge movement and for the sliding filament model in actively contracting skeletal muscle. J. Mol. Biol. 77:549-568. 1973.

Hasselgrove, J.C. Structure of wertebrate striated muscle determined by $X$-ray-diffr action studies. In: Handbook of Physiology. Section 10: Skeletal muscle. Eds.: Peachey, L.D., H.A. Adrian and S.R. Geiger. American Physiological Society (Bethesda, Maryland). 143-171. 1983.

Havenith, M.G., F.H. wan der Veen, J.F.C. Glatz, C. Lucas, J.M.C. Schrijvers-van Schendel, O.C.K.M. Penn and H.J.J. Wellens. Monitoring of muscle fiber type of canine latissimus dorsi muscle during chronic electrical stimulation by enzyme- and immunohistochemistry. In. Transformed muscle for cardiac assist and repair. Futura, New York. (in press). 1989.

Henneman, E., and C.M. Olson. Relations between structure and function in the design of skeletal muscles. J. Neurophysiol. 28:581-598. 1965.

Herrmann, K.S. Platelet aggregation induced in the haraster cheek pouch by a photochemical process with excited fluorescein isothiocyanate-dextran. Microwasc. Res. 26:238-249. 1983.

Higuchi, $H$., and $Y$ : Umazume. Lattice shrinkage with increasing resting tension in stretclied, single skinned fibers of frog muscle. Biophys. J. 50(3):385-389. 1986.

Holthöfer, H., I. Virtanen, A.-L. Kariniemi, M. Hormia, E. Linder and A. Miettinen. Ules europaeus I lectin as a marker for vascular endothelium in human tissue. Laboratory Investigation 47(1),60 66. 1982 .

Hoppeler, H. Exercise-induced ultrastructural changes in skeletal muscle. Int. J. Sports Med. 7:187204. 1986.

Hoppeler, $\mathrm{H}$. The structural composition of muscle tissue and its changes with different types of exercise. Geneeskunde en Sport 20(2):56-64. 1987.

Howald, H, H. Hoppeler, H. Claassen, O. Mathieu and R. Straub. Influences of endurance training on the ultrastructureal composition of the different muscle fiber types in humans. Pfiugers Arch. 403:369-376. 1985.

Hudlická, O., L. Dodd, E.M. Renkin and S.D. Gray. Early changes ün fiber profile and capillary density in llong term stimulated muscles. Am. J. Physiol. 243:H528-H535. 1982.

Huxley, H.E. Structural difference between resting and rigor muscle; evidence from intensity changes in the low-angle equatorial X-ray diagram. J. Mol. Biol. 37.507-520. 1968.

Inger, $F_{,}$, and P. Brodal. Capillary supply of skeletal muscle fibers in untrained and endurance-trained women. Europ. J. Appl. Physiol. 38:291-299. 1987. 
Intaglietca M. and W.R. Tompkins. Microvascular measurements by video image shearing and splitting. Microvasc. Res. 5:309-312 1973.

Ishiharaima, S., T. Aida, R. Nakagawa, K. Kameyama, K. Sugano, T. Oguro and G. Asano. Early membrane damage during ischemia in rat heart. Exp. Mol. Pathol, 44:1-6. 1986.

Isobe, $Y, F, D$. Warner and L.F. Lenianski. Three-dimensional immunogold lacalization of alpha-actinin within the cytoskeletal networks of cultired cardiac muscle and nonmuscle cells. Proc. Natl. Accad. Sci. 85:6758-6762. 1988.

Janssens C.J J G. R.S. Reneman, D.W. Slaaf and G.-J. Tangelder. Differentiation in wivo of leukocytes rolling in mescnteric wenules of anaesthetised rabbits. J. Physiol Abstract. Submitted. 1989.

Jerusalem, F. A.G. Engel, and M.R. Gomez. Duchenne dystrophy. Brain 97:115-122. 1974A.

Jerusallem, F. M. Rakusa, A.G. Engel and R.D. Macdonald. Morphometric analysis of skeletal muscle capillary ultrastructure in inflammatory myopathies. J. Neurol. Sci. 23.391-402. 1974B.

Katufman, A.G. and M. Intagtietta. Autonated diameter measurement of vasomotion by cross-correlation. Int. J. Microcirc: Clin. Exp. 4:45-53. 1985.

Khaw, B., J.A. Mattis, G. Melincoff, H.W. Strauss, H.K. Gold, and E. Haber. Monoclonal antibody to cardiac myositi: imaging of experimental cardiac infarction. Hybridoma 3(1):11-23. 1984.

Kowacs, I.B., A. Tigy"-Sebers, K. Trombit terial to produce intravital microinjury by He-Ne gas laser. Microvasc. Res. 10:107-124. 1975.

Kuipers, H., J Drukker, P.M. Frederik, P. Geurten and G. Kranenburg v. Muscle dlegeneration after exercise in rats. Int. J. Sports Med, 4:45-51. 1983.

Kuipers, H. H.A. Keizer, F.T.J. Verstappen and D.L. Costill. Influence of a protaglandin-inhibiting drug on muscle soreness after eccentric work. Int. J. Sports Med. 6:336-339. 1985.

Laughlin, A. and R.B. Armstrong. Rat muscle blood flows as a function of time during prolonged slow treadmill exercise. Am. J. Physiol. 244:H814-H824. 1983.

Lindholm, A., H. Johansson and P. Kjorsgaard. Acute Rhabdonyolysis ("tying-up") in standardbred horses. Acta. Vet Scand. 15:325-339. 1974.

Little, D., J.W. Said, R.J. Siegel, M. Fealy and M.C. Fishbein. Endothelial cell markers in vascular neoplasms: an immunohistochemical study comparing factor VIII-related antigen, blood group specific antigens, 6xketo-PGF1 alpha, and Ulex europaeus 1 lectin. J. Pathol. 149:89-95. 1986.

Lowey, S., and D. Risby. Light chains from fast and slow muscle myosins. Nature. 234:81-85. 1971.

Lucas S.M., R.L. Ruff, and M.D. Binder. Specific tension measurements in single soleus and medial gastrocnemius muscle fibers of the cat. Exp. Neurol. 95:142-154. 1987.

Luginbuhl, A.J., G.A. Dudley and R.S. Staron. Fiber type changes in rat skeletal muscle after intense interval training. Histochemistry 81:55-58. 1984.

Mahler, $F, G, N_{2}$ agel, H. Saner, and F. Kneubihl in wivo comparison of nailfold capillary diameter as determined by using the erythrocyte colum and FITC-labeled albumin. Int, J. Microcirc: Clin. Exp. 2:147-155. 1983.

Mater , A., L. Gorza, S. Schiaffino and D. Pette. A combined histochemical and immunohistochemical study on the dymanics of fast-to-slow fiber transformation in chronically stimulated rabbit muscle. Cell Tissue Res. 254:59-68. 1988.

Makitie, J. Microvasculature of rat striated muscle after temporary ischemia. Acta Newropathol. $37247-253.1977$.

Makkitie. J. and H. Teräwäinen. Ultrastructure of striated muscle of the rat after temporary ischemia. Acta Neuropathol. 37:237-245. 1977.

Matsen, F.A. Compartmental syndrome. Clin. Orthop. 113:8-14. 1975.

Matsubara ${ }_{2}$., and G.F. Elliot. X-ray diffraction studies on skinned single fibers of frog skeletal muscle. J. Mol. Biol. 72:657-669. 1972.

Matsubara, $I_{n}$, Y.E. Goldman, R.M. Simmons. Changes in the lateral filament spacing of skinned muscle fibers when cross-briclges attach. J. Mol. Biol. 173(1):15-33, 1984A.

Matsubara, $I_{2}, Y$. Umazume and $\mathbb{N}$. Yagi. Lateral shrinkage of the myofilament lattice in chemically skinned muscle during contraction. Adv. Exp. Med. Biol. 170:711-720. 1984B.

Maughan, D.W. and R.E. Godt. Stretch and radial compression studies on relaxed skinned muscle fibers of the frog. Biophys. J. 28:391-402. 1979. 
Maier, A., L. Gorza, S. Schiaffino and D. Pette. A combined histochenical and inimunohistochentcall study on the dynamics of fast-to-slow fiber transformation in chronically stimulated rabbit muscle. Cell Tissue Res. 254:59-68. 1988.

McCully, K.K., and J.A. Faulkner. Characteristics of Jengthening contractions associated with injury to skeletal muscle fibers. J. Appl. Physiol. 61(1):293-299. 1986.

Metzger, J.M., and R.L. Moss. Shortening velocity in skinned single muscle fibers. Influence of fillament lattice spacing. Biophys. J. 52(1):127-131. 1987.

Moneta, G., M. Brülisauer, K. Jäger and A. Bollinger. Infrared fluorescence videomicroscopy of skin capillaries with indocyanine green. Int. J. Microcirc: Clin Exp. 6:25-34. 1987.

Moore, S.E., O. Hurko and F.S. Walsh. Immunocytochenical analysis of fiber type differentiation in developing skeletal muscle. J. Neuroimmunal, 7:137.149. 1984.

Myrhage, $R_{\text {, }}$ and $O$. Hudlicka. The microvascular bed and capillary surface area in rat extemsor hal. lucis proprius muscle (EHP). Microvasc. Res. 11:315-323. 1976.

Myrhage, R. Capillary supply of the muscle fiber population in hindlimb muscles of the cat. Acta Physiol. Scand. 103:19-30. 1978.

Nemeth, P., H.-W. Hofer and D. Pette. Metabolic heterogeneity of muscle fibers classilied by myosin ATPase. Histochemistry 63:191-201. 1979.

Nemeth $P$. and D. Pette. Succinate dehydrogenase activity in fibres classified by myosin ATPase in three hind limb muscles of rat. J. Physiol. 320:73-80. 1981.

Ninomiya, T., H. Yosimura and M. Mori. Identification of vascular system in experimental carcinoma for cryosurgery - Histochemical observations of tectin UEA-1 and alkaline phosphat ase activity in vascular endothelium. Cryobiology 22:331-335. 1985.

Orgnero de Gaisan, E., A. Aokt, D. Heinrich and J. Metz. II. Tracer permeation and freeze-fracture of fetal endothelium. Anat. Embryol. 171: 297-304. 1985.

Peeze Binkhorst, F.M., H. Kuipers, J. Heymans, P.M. Frederik, D.W. Slaaf, G.-J. Tangelder and R.S. Reneman. Exercise-induced focal skelecal muscle fiber degeneration and capillary morphology. I. Appl. Physiol , 66(6):2857-2865. 1989A.

Peeze Binkhorst, F.M., H. Kuipers, G.-J. Tangelder, P.M. Frederik, D.W. Slate and R.S. Reneman. Capillary (ultra)structure in relation to position and fiber type in rat soleus muscle. Submitted. 1989:

Peter, J.B., R.J. Barnard, V.R. Edgerton, C.A. Gillespie and K.E. Stempel. Metabolic profiles of three fiber types of skeletal muscle in guinea pigs and rabbits. Biochemistry 11.(14):2627-2633. 1972.

Pierobon-Bormioli, S., S. Sartore, L. Dalla Libera, M. Vitadello and S. Schiaffino. "Fast" isornyosins and fiber types in mammalian skeletal muscle. J. Histochem. Cytochem, 29(10):1179.1188. 1981.

Pietra, G.G., A.P. Fishman, F.N. Lanken, P. Sampson and J. Hansen-Flaschen. Anntals of the New York Academy of Sciences. New York. The New york Acaderny of Sciences. 240-247. 1982.

Potter, R.F.s and A.C. Groom. Capillary diameter and geometry in cardiac and skeletal muscle studied by means of corrosion casts. Microvasc. Res. 25:68-84. 1983.

Pullen, A.H. The distribution and relative size of fiber types in the extensor digitorum longus and soleus muscles of the adult rat. J. Anat. 123(2):467-486. 1977.

Reed, M.W.R., and F.M. Miller. Importance of light dose in fluorescent microscopy. Microvasc. Res. 36:104-107. 1988.

Reinhold, H.S, and J.W.M. Visser in wivo fluoreseence of endothelial cell nuclei stained with the dye Bis-benzamide H 33342. Int. J, Microcirc: Clin. Exp. 2:143-146. 1983.

Reneman, R.S. The anterior and lateral compartment syndrome of the leg. Mouton \& Co. The HagusParis. 176 pag. 1968.

Reneman, R.S. The anterior and lateral compartmental syndrome of the leg due to intensive use of muscle. Clin. Orth. 113:69-80. 1975.

Reneman, R.S., D.W. Slaaf, L. Lindbom, G.-J. Tangelder and K.-E. Arfors. Mfuscle blood now disturbances produced by simultaneously elevated venous and total muscle tissue pressure. Microvasc. Res. 20:307-318. 1980.

Rosenblum, W.I., and F. El-Sabban. Platelet aggregation in the cerebral microcirculation. Circ. Res. $40(3): 320-328.1977$. 
Rubinstein, $\mathrm{N}_{\mathrm{i}}$ K. Mabuchi, F. Pepe, S. Salmons, J. Gergely and F. Sreter. Use of type-specific antimyosing to demonstrate the transformation of individual fibers in chronically stimulated rabbit fast muscles. J. Cell Biol. 79:252-261. 1978.

Ryan, U.S, J,W. Ryan, C. Whitaker and A. Chiu. Localization of angiotensin converting enzyme (kitaase II). II. Immunocytochemistry and immunofluorescence. Tussue Cell 8(1):125-145. 1976.

Salminen, $A$, and V. Vihko. Susceptibility of mouse skeletal muscles to exercise injuries. Muscle and Nerve. 6:596-601. 1983.

Salminen, $A_{,}$and $V$. Vihko. Autophagic response to strenous exercise in mouse skeletal muscle fibers. Virchow: Arch. (Cell Pathol) 45:97-106. 1984A.

Salminen, $A_{m}, K$. Hongisto and V. Vihko. Lysosomal changes related to exercise injuries and traininginduced protection in mouse skeletal muscle. Acta Physiol. Scand. 120:15-19. 1984B.

Salmons, S., D.R. Gale and F.A. Sreter. Ultrastructural aspects of the transformation of muscle fiber type by long term stimulation: changes in $Z$ discs and mitochondria. J. Anat. 127(1):17-31. 1978.

Sanderson, R.A., R.K. Foley, G.W.D. Mclvor and W.H. Kirkaldy-Willis. Histological response on skeletal muscle to ischemia. Clin. Orthop. 113:27-35. 1975

Sartore, \$. Immunological cross-reactivity between chicken slow skeletal and ventricular muscle myosin. Biochimica et Biophysica Acta. 667:143-156, 1981.

Sato $M$. and $N$, Ohshima. Platelet thrombus induced in vivo by filtered light and fluorescent dye in mesenteric microvessels of the rat. Thromb. Res. 35:319-334. 1984.

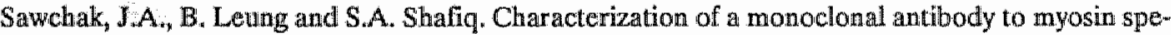
cific for mamalian and human type II muscle fibers. I. Neurol. Sci. 69:247-254. 1985.

Sethlegel, J.U. Demonstration of blood vessels and lymphatics with a fluorescent dye in ultraviolet light. Anat. $\operatorname{Rec}_{\text {. 105:433-443. } 1949 .}$

Sclalingemann, R.O., G.M. Dingjan, J.J. Emeis, J. Blok, S.O. Warnaar and D.J. Ruiter. Monoclonal nutibody PAL-E specific for endothelium. Lab. Invest. 52(1):71-76. 1985.

Schmid-Schömbein, G.W., R. Skalak, S. Usami and S. Chien. Cell distribution in capillary networks. Microvas. Res. 19:18-44. 1980.

Sclaneeberger, E.E of $_{\text {and }}$ M.J. Karnovsky. The influence of intravascular fluid volume on the permeability of newborn and adult mouse lungs to ultrastructural protein tracers. J. Cell Biol. 49:319334. 1971.

Schwane, J.A., S.R. Johnson, B. Vandenakker and R.B. Armstrong. Delayed-onset muscular soreness and plasma CPK and LDH activities after downhill running. Med. Sci. Sports 15(1): 51-56. 1983.

Siegel, A.J., L.M. Silverman, B.L. Holman. Elevated creatine kinase MB isoenzyme levels in marathom runners. JAMA 246:2049-2051. 1981.

Simionescu, N., M. Simionescu, and G.E. Palade. Permeability of intestinal capillaries. J. Cell Biol. $53: 365-392,1972$

Simionescu, N., M. Simionescu and Palade G.E. Permeability of muscle capillaries to exogenous. myoglobin. J. Cell Biol. 57:424-452. 1973.

Simionescu, N. M. Simionescu and G.E. Palade. Permeability of muscle capillaries to small hemepeptides. J. Cell Biol. 64:586-607. 1975.

Sünionescu M., N. Simionescu and G.E. Palade. Differentiated microdomains on the luminal surface of capillary endothelium. I. Prelerential distribution of anionic sites. J. Cell Biol. 90:605-613. 1981A

Simionesen M., N. Simionescu, J. Silbert and G.E. Palade. Differentiated microdomains on the luminal surface of capillary endothelium. II. Partial characterization of their anionic sites. J. Cell Biol 90:614-621. 1981B.

Simionescu, M., N. Simionescu and G.E. Palade. Differentiated microdomains on the luminal surface of capillary endothelium: Dstribution of lectin receptors. J. Cell Biol. 94:406-413. 1982.

Sjöström, $M_{\text {., }}$ J. Fridén and B. Ekblom. Fine structural datails of human muscle fibers after fiber type specific glycogen depletion. Hustochemistry. 76:425-438. 1982.

Slaaf, D.W., R. Alewijnse and H. Wayland. Use of telescopic imaging in intravital microscopy: A simple solution for conventionall microscopes. Int. J. Microcirc: Clin Exp. 1:121-134. 1982.

Smith, D, H. Green, J. Thomson and M. Sharratt. Capillary and size interrelationship in developing rat ditaphragm, EDL and soleus muscle fiber types. Am. J. Physiol. 256 (1):C50-C58. 1989. 
Smith, $\mathrm{H}_{\text {, }}$ and T.C. Jones. The musculoskeletal system. In: Veterinary Pathology. Lea and Febriger, Philadelphia. 812-815. 1966.

Spurway, N. Interrelation between myosin-based and metabolism-bused classification of skeletal muscle fibers. J. Histochem. Cytochem. $29(1): 87-90.1981$.

Squire, J. The structural basis of muscular contraction. Plenum Press New York and London. 334340. 1981.

Staron, R.S., R.S. Hikida, F.C. Hagerman, G.A. Dudley and T.F. Murray. Human skeletal muscle fiber rype adaptability to various workloads. J. Histochem. Cytochem. 32(2):146-152. 1984.

Staron, R.S., and D. Pette. Correlation between myofibrillar ATPase activity and rayosin heavy chain composition in rabbit muscle fibers. Histochemistry 86:19-23. 1986.

Stauber, W.T., V.K. Fritz, D.W. Vogelbach and B. Dahimann. Characterization of muscle injured by forced lengthening. I. Cellular infiltrates. Med. Sci. Sports Exerc. 20(4):345-353. 1988.

Sullivan, S.M., and R.N. Pittman. In vitro 02 uptake and histochemical fiber type of resting hamster muscles. J. Appl. Physiol.: Respirat. Environ. Exercise Physiol. 57(1):246-253. 1984.

Tangelder, G.J., D.W. Slaaf and R.S. Reneman. Fluorescent labeling of blood platelets in wivo. Thromb. Res. 28:803-820. 1982.

Tangelder, G.J., D.W. Slaaf and R.S. Reneman. Skeletal muscle microcirculation and changes in transw mural and perfusion pressure. Prog. Appl. Microcirc 5:93-108. 1984.

Thornell, L.-E., E. Carlsson, E. Kugelberg and B.K. Grove. Myofibrillar M-band structure and composition of physiologically defined rat motor units. Am. J. Physiol. 253:C456-C468. 1987.

Tyml, $\mathrm{K}_{\text {. }}$ and $\mathrm{H}$. Weigelt. Contribution of the capillary contractility to the distribution of microflow in skeletal muscle. Int. J. Micracirc.: Clin. Exp. 1:331-332. 1982.

Tornling, $\mathrm{G}$. Capillary neoformation in the heart and skeletal muscle during diprydanole-treatment and exercise. Acta Pathol. Microbiol. Immunol. Scand. Section A, supplements 278. 1-63. 1982.

Voyta, J.C. D.P. Via ${ }_{3}$ C.E. Butterfield and B.R. Zetter. Identification and isolation of endothelial cells based on their increased uptake of acetylated-Iow density lipoprotein. J. Cell Biol. 99:2034-2040. 1984.

Wang, K., and J. Wright. Architecture of the sarcomere matrix of skeletal muscle: Immunoelectron microscopic evidence that suggests a set of parallel inextensible nebulin filaments anchored at the Z line J. Cell Biol. 107(6):2199-2212. 1988.

Warhol, M.J., A.J. Siegel, W.J. Evans and L.M. Silyerman. Skeletal muscle injury and repair in marathon runners after competition. Am. J. Pathol 118 331-339. 1985.

Wayland, H., and P.C. Johnson. Erythrocyte velocity measurement in microvessels by a two-split photometric method. J. Appl. Physiol. 22:333 337. 1967.

Weigelt, H., T. Fujii, D.W. Lubbers and G. Hauck. Specialized endothelial cells in the frog mesentery - attempt of an electrophysiological characterization. Bibl. anat. 20:89-93. 1981..

Whalen, R.G., K. Schwartz, P. Bouveret, S.M. Sell and F. Gros. Contractile protein isozymes in muscle dewelopment: Identification of an embryonic form of myosin heavy chain. Proc. Natt. Acad. Sci. USA. 76(10):5197.5201. 1979.

Wiederbielm, C.A., J.W. Woodburry, S. Kirk and R.F. Rushmer. Pulsatile pressures in the microcinculation of frog's mesentery. Am. J. Physiol. 270(1):173-176. 1964.

Wiederhielm, C.A. The tissue pressure controversy, a semantic dilemma. In: Tissum flüd pressure and composition. Ed.: Hargens, A.R. Williams and Wilkins, Baltimore/London. 21-33. 1981.

Wiederhielm, C.A., and D.W. Slaaf. A new skeletal muscle preparation fior the study of microvascular function in intact unanesthetized animals. Microvasc. Res. 33:413-4:16. 1987.

Wiig, H., R.K. Reed and K. Aukland. Micropressure measurements of interstitial fluid pressure in rat subcutis and skeletal muscle: comparison to wick in meedle technique. Microvasc. Res. 21:308-319, 1981.

Witte, S. Surface phenomena of coagulation factors in viwo, observed by fluoresce nce intravital microscopy. Ann. NY Acad. Sci, 426-440. 1983.

Yokota, S. Imnunocytochemical evidence for transendothelial transport of alburnin and fibrinogen in rat heart and diaphragm. Biomed. Res. 4(6):577-586. 1983. 


\section{SUMMARY}

It has been shown that uphill endurance running by untrained rats can induce segmental degeneration of individual muscle fibers. The degeneration is characterized by infiltration of inflammatory cells which is most explicit $24-48 \mathrm{~h}$ after running. Because this fiber damage after unaccustomed exercise appears to be similar to that observed following ischemia, impairment of the microcirculation might contribute to the exercise induced fiber damage.

The aim of the present study was to obtain insight into the role of ischemia, if any, in exercise induced muscle fiber degeneration. More specifically we investigated:

- whether muscle fiber degeneration is preceeded by capillary morphological changes indicative of ischemia. Morphological analysis of capillary cross-sections at the light and electron microscopic level was used to detect possible capillary obstructions and to search for general and local capillary abnormalities in exercised rats and in control rats that did not run.

- whether slow twitch (type I) fibers of the soleus muscle, that are more vulnerable to exercise than fast twitch (type II) fibers are supplied by fewer capillaries or by capillaries that are morphologically less well adapted to efficient exchange of substances.

- whether exercise induced muscle fiber degeneration is preceeded by swelling of muscle fibers and, if so, whether this swelling leads to an increase in tissue pressure to a level sufficiently high to impair blood flow.

The studies were performed on the soleus muscle of untrained rats. To induce muscle overload, rats ran for $75 \mathrm{~min}$ on a rodent treadmill with a positive incline of $10^{\circ}$ at a speed of approximately $1.5 \mathrm{~km} / \mathrm{h}$. After running, muscles were fixated in situ by perfusion to preserve capillary and muscle fiber (ultra)structure. To investigate capillary morphology in relation to fiber type, muscle fibers in plastic cross-sections were classified, based upon myosin ATPase activity, as type I (slow twitch) or type II (fast twitch) fibers by comparing them with their counterparts that were immunologically classified in dewaxed cross-sections (retrieval method). Whenever myosin ATP-ase activity had to be preserved, e.g. to check the reactivity of the antibody, or when tissue shrinkage had to be minimized, e.g. to determine muscle swell- 
ing after running cryofixation of muscles was used. Since swelling of muscle was observed following running and this swelling might have increased tissue pressure, resulting in decreased muscle perfusion, intramuscular pressure was measured using a servo controlled backpressure system. The methods used are described in chapters 3,4 and 5 .

Following running, the degenerated muscle fibers were situated between fibers with a normal morphological appearance at the light as well as at the electron microscopic level Capillaries appeared to be unaffected by the running procedure. Their lumina were free of blood cells and clusters of fixated plasma proteins as a result of the flushing during perfusion fixation. The overall morphology of the capillaries and their dimensions were normal. However, degenerated fibers were supplied by capillaries with a $50 \%$ larger luminal cross-sectional area than the more distant capillaries, but their morphology was normal and their lumina were free of obstructions. This indicates that the degenerated fibers were well supplied with blood (chapter 3).

In the soleus muscle, type $\mathbb{I}$ as well as type Ir fibers are present. However, the degenerated fibers are always of type I. In the soleus muscle, type I and type II fibers are supplied by equal numbers of capillaries of similar dimensions. This suggests that capillaries, which supply the degenerated fibers, had normal dimensions before degeneration started and that they are dilated due to the degenerative process. In addition, it could be demonstrated that, independent of fiber type, capillaries situated at the corner of three or more fibers have a $27 \%$ larger cross-sectional area than those situated between two fibers, a location where less space is available (chapter 4).

Transient swelling of the muscle fibers as a result of running was observed. The increase in muscle and muscle fiber volume was largest 6 h after running (20-30\%; chapter 3 and 5). Interestingly, the swelling did not cause an increase in intramuscular pressure, which remained at the control value of about $1 \mathrm{~mm} \mathrm{Hg}$ in the observation period between 18 min and 24 hours after running. Hence, post-exercise swelling of the muscle does not lead to prolonged increases in intramuscular pressure to a level high enough to cause an impaired muscle perfusion (chapter 5).

During the classification of muscle fibers in plastic cross-sections it was observed that in the soleus muscle type I fibers stained darker than type II fibers. This finding suggests a difference in contractile filament spacing between slow twitch and fast twitch fibers. The intensity of staining of muscle fibers in cross-sections with toluidine blue correlated significantly with the spacing of the thick filaments. Spacing of thick filaments was $4-9 \%$ smaller in slow twitch than in fast twitch fibers with no overlap in the soleus, and little overlap in the gastrocnemius and peroneus muscles (chapter 6).

In the chapters 7 and 8 an attempt was made to study capillary morphology in muscle in wivo.

In chapter 7, fluorescent dyes were tested for their ability to label blood plasma, allowing visualization of capillaries, or endothelium in the mesentery and soleus muscle of the rat in vivo. Apart from the generally used FITC-Serum Albumin, the 
FITC-lectins tested appeared to be good candidates for fluorescent labeling of blood plasma. Of all dyes tested, only Pyronin B labeled the endothelium of muscle capillaries satisfactory. Labeling was temporarily and injection of the dye could cause a considerable drop in systemic blood pressure and capillary flow. With the method used in this study, systemic blood pressure and capillary flow return to about $75 \%$ of their initial values approximately 3 minutes after the first injection and remain approximately at that level throughout the observation period. Acridine yellow accumulated in nuclei, among others of endothelial cells and leukocytes. Some dyes, mainly the ones that labeled blood platelets and/or endothelial cells, were found to be potent stimulators of platelet aggregation upon excitation.

In the final experimental chapter 8, fluorescent labeling of the capillary lumina and image analysis were used to determine capillary inner diameters in wivo. The average inner diameters of capillaries, as determined in plastic sections (chapters 3 and 4) were about $26 \%$ smaller than those assessed in vivo. This might, at least in part, be explained by tissue shrinkage during fixation, dehydration and/or embedding of the tissue.

In conclusion, we found no support for the hypothesis that ischemia is the primairy cause of exercise induced muscle fiber degeneration. Following running, no changes in the capillary morphology indicative of ischemia are observed. Affected and vulnerable muscle fibers are supplied by a normal number of capillaries with a normal morphology. Affected fibers are supplied by dilated capillaries and are surrounded by morphologically unaffected fibers. Tissue pressure does not change following running and, hence, cannot have impaired muscle perfusion. 



\section{SAMENVATTING}

Spieren gebruiken wij alsof het de normaalste zaak van de wereld is. Mogelijk houden uw spieren op dit moment uw vingers om dit boekje geklemd. Spieren van uw oog bewegen uw blik over deze en de volgende regels. Uw ademhaling wordt door spieren op gang gehouden en uw hartspier pompt bloed naar de spieren zodat deze hun werk kunnen blijven doen. Tal van zaken zijn van invloed op het functioneren van de spier. De anatomie (opbouw) en de fysiologische eigenschappen van een spier bepalen voor een deel de mogelijkheden. De doorbloeding van een spier is van invloed op het uithoudingsvermogen ervan. Een spier kan zich door traïning aanpassen aan nieuwe belastingen. Indien echter een belasting te groot wordt (overbelasting) kan spierbeschadiging optreden.

De onderzoeken, die beschreven staan in dit proefschrift, hebben betrekking op skeletspieren en de kleinste bloedvaatjes daarvan. In het bijzonder is onderzocht of de spierbeschadigingen die ontstaan na overbelasting (zie hierna) mogelijk veroorzaakt worden door een slechte spierdoorbloeding.

In deze Nederlandstalige samenvatting zullen de anatomie, fysiologie en doorbloeding van de skeletspier kort behandeld worden. Daarna worden de opzet van het onderzoek en tenslotte de belangrijkste resultaten van het onderzoek besproken.

\section{DE SKELETSPIER}

Anatomie van de skeletspier.

Ongeveer de helft van ons lichaam bestaat uit skeletspieren. Zoals de naam al aangeeft kunnen skeletspieren het skelet en zodoende ook het lichaam doen bewegen. Een skeletspier is, zoals alle organen van ons lichaam, opgebouwd uit cellen. De meeste cellen van een skeletspier zijn versmolten tot lange dunne vezels, de zgn. spiervezels (figuur 12.1 en 12.4). Deze spiervezels kunnen in lengterichting 

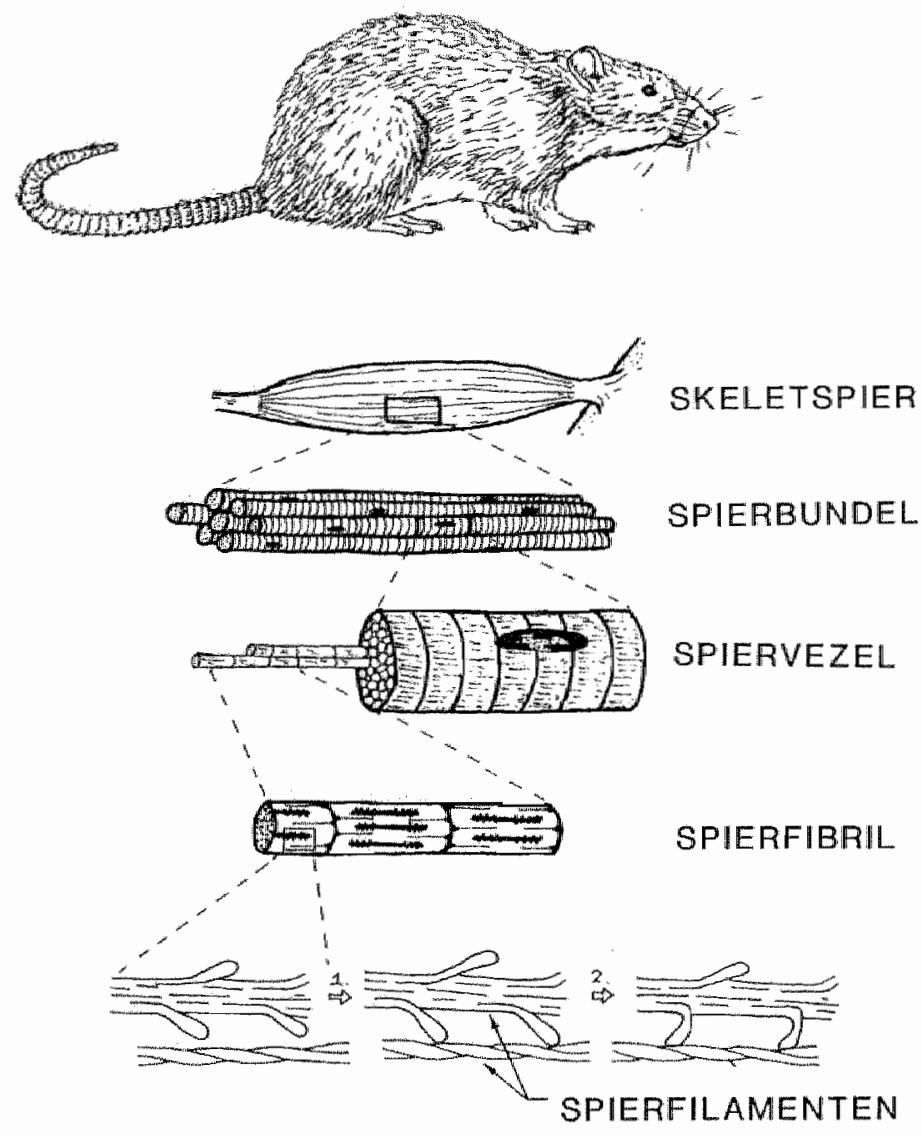

Fig. 12.1. Anatomie van de skeletspier. De spierfilamenten kunnen langs elkaar glijden waardoor de spiervezels en de spier kunnen samentrekken.

samentrekken. Ze zijn ongeveer $1 / 20 \mathrm{~mm}$ dik en kunnen even lang zijn als de spier zelf. In de spiervezels zelf bevinden zich weer parallel lopende spierfilamenten (zie figuur 12.t en evt. liguur 6.2A, blz. 66) die langs elkaar kunnen glijden. Hierdoor kunnen de spiervezels, en dus de spier, samentrekken. Niet alle spiervezels zijn gelijk. Er zijn spiervezels die langdurig gebruikt kunnen worden zonder dat zij vermoeid raken. Deze zgn. langzame spiervezels (slow twitch of type I vezels) vinden we o.a. in relatief grote aantallen in spieren die gebruikt bij het in stand houden van het lichaamsevenwicht. Ze zijn relatief traag en niet erg sterk. Snelle spiervezels (fast twitch of type II vezels) daarentegen zijn relatief snel en kunnen grotere krachten ontwikkelen. Ze zijn m.n. aanwezig in spieren die actief bij activiteiten zoals sprinten of gewichtheffen. Met speciale kleuringstechnieken zijn snelle en langzame spiervezels te onderscheiden (zie evt. figuur 4.2, blz. 37). Er zijn ook nog an- 


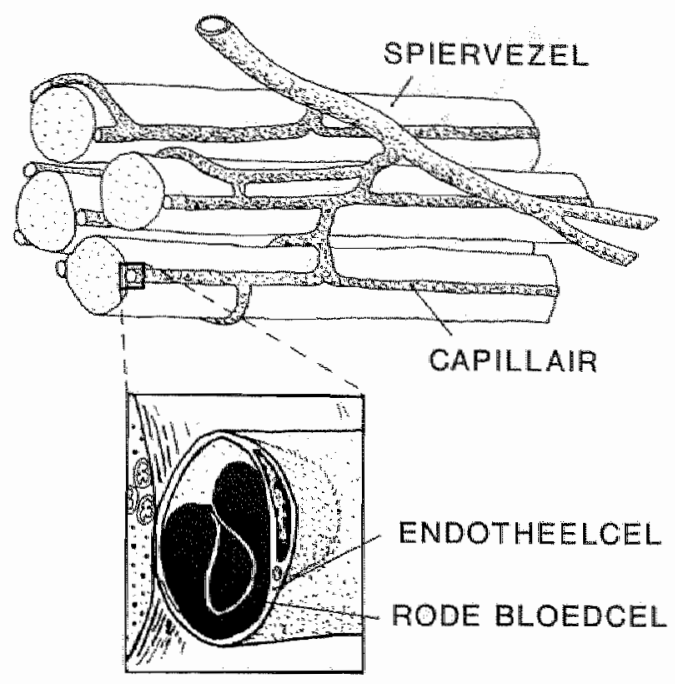

Fig. 12.2. De capillaire voorziening van de spiervezels. De capillairen lopen voornamelijk parallel aan de spiervezels. Indien een spier verkort is lopen de capillairen meestal gekronkeld langs de spiervezels. In het kader is een dwars doorgesneden capillair vergroot weergegeven. Op het snijvlak zijn een platte endotheelcel en een rode bloedcel te zien.

dere manieren om spiervezels te onderscheiden die hier echter niet aan de orde zullen komen.

De doorbloeding van de skeletspier.

Het samentrekken van een spier kost energie. Die energie komt vrij door de verbranding van m.n. suiker en vet in de spiervezels. Wil een spier blijven werken dan moeten er, net zoals bij een benzinemotor, continu brandstoffen worden aangcvoerd en afvalprodukten worden afgevoerd. Dit gebeurt via het bloed. Het bloed neemt zuurstof op uit de longen en brandstof uit de darmen. Via het hart en de slagaders bereikt het bloed de spier. De slagaders vertakken verder tot kleine slagdertjes (arteriolen) die door samenknijpen of ontspannen de doorbloeding van een bepaald spiergebied kunnen reguleren. De arteriolen vertakken weer in de nog kleinere haarvaatjes oftewel capillairen (figuur 12.2 en evt. figuur 4.1, blz 36). Dit zijn de kleinste bloedvaatjes die we in de spier aan treffen. Ze zijn zo klein dat de meeste bloedcellen die er doorheen stromen worden vervormd. De wand van de capillairen is uiterst dun en bestaat uit slechts eén laag van platte cellen, de endotheelcellen, die tesamen een buisje vormen. Stoffen kumnen via de dunne wand van de capillairen snel worden uitgewisseld tussen het bloed en de spiervezels. Indien 
een spiervezel door slechts weinig capillairen is omgeven, kan deze bij inspanning te weinig zuurstof krijgen waardoor de spiervezel bij aktiviteit snel vermoeid raakt. Spieren die langdurig moeten werken zijn dan ook altijd goed woorzien van capillairen om over voldoende zuurstof te beschikken en hun afvalprodukten snel kwijt te kunnen raken. Het bloed wordt vanuilt de capillairen afgevoerd via bloedvaten naar het hart.

\section{Aanpassingsvermogen van de skeletspier}

Het is duidelijk dat spieren zich kunnen aanpassen aan veranderende omstandigheden. Door training kunnen spieren sterker (boxers), dikker (body builders) of sneller (sprinters) worden of een groter uithoudingsvermogen ontwikkelen (marathonlopers). De aanpassing van de spier wordt voor een belangrijk deel veroorzaakt door veranderingen van de spiervezels die niet alleen in aantal kunnen toenemen. maar ook van afmeting en eigenschappen kunnen veranderen. Door training kan bovendien het aantal capillairen van de spier toe of afnemen.

De aanpassing van een spier is echter niet grenzeloos. Vrijwel iedereen kent het verschijnsel van spierpijn na een zware en/of langdurige inspanning die men niet gewend is. Wat precies de oorzaak van die pijn is, is nog onbekend. In ieder geval wijst spierpijn er op dat er iets in de spier veranderd is. Met behulp van microscopisch onderzoek is aangetoond dat sommige spiervezels door zware of langdurige inspanning plaatselijk beschadigd en ontstoken kunnen raken. Meestal is de ontstekingsreactie na éen tot enkele weken weer volkomen genezen. In uitzonderlijke gevallen echter kunnen uit de beschadigde spiervezels spiereiwitten vrijkomen die de nier beschadigen en soms zelfs de dood tot gevolg kunnen hebben. Dit is niet alleen aangetoond bij dieren, zoals bijvoorbeeld renpaarden, maar ook bij mensen.

\section{HET ONDERZOEK.}

De precieze oorzaak van de spiervezelbeschadiging die ontstaat na overbelasting is nog niet bekend. De beschadigingen lijken sterk op de ontstekingen die ontstaan indien de bloedtoevoer naar een spier wordt afgesloten. De lengte waarover de spiervezels beschadigd raken is ongeveer gelijk aan de lengte van de capillairen die de spiervezels van bloed voorzien. Dit wijst er op dat de spiervezelbeschadiging ontstaat door een slechte doorbloeding van bepaalde capillairen. In dit onderzoek is in de eerste plaats nagegaan of de spiervezelontstekingen inderdaad veroorzaakt worden door een belemmerde doorbloeding van die capillairen.

In alle experimenten is de rat als proefdier gebruikt. Om een ontstekingsreactie in de spiervezels op te wekken moesten de ratten éenmalig gedurende 5 kwartier op een loopband rennen. De loopband kreeg een positieve helling van $10^{\circ}$ en werd ingesteld op een snelheid van $1,5 \mathrm{~km} /$ uur. De spier die door ons als model werd 


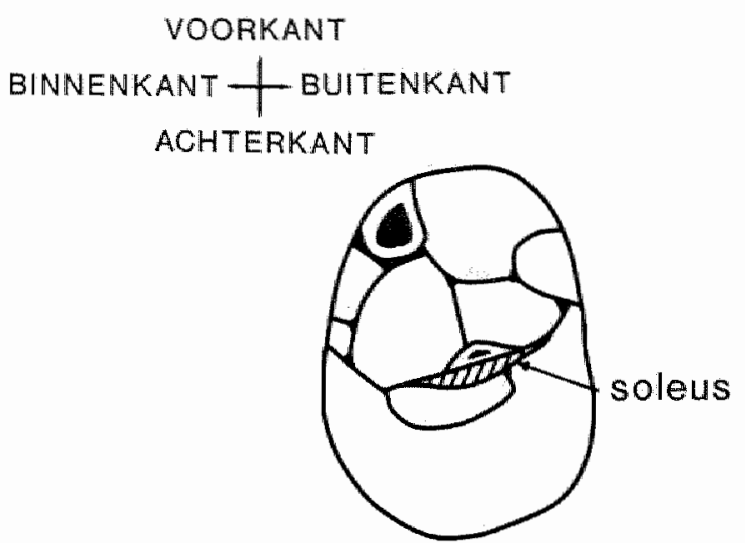

Fig. 12.3. Dwarsdoorsnede door de rechter achterpoot van de rat ter hoogte van de kuit. De soleus spier ligt vrijwel centraal in het onderbeen en is betrokken bij de beweging van de voet.

gekozen was de scholspier oftewel soleus spier. Deze spier ligt diep in de kuit ligt (figuur 12.3) en is relatief erg gevoelig voor het tegen een helling op rennen.

Met behulp van microscopisch onderzoek zijn de veranderingen onderzocht die in de capillairen van spieren optreden na hardlopen. Daarbij is gebruik gemaakt van licht- en, voor meer gedetailleerd onderzoek, electronenmicroscopen. $\mathrm{Er}$ is gekeken of de capillairen zodanig verstopt of beschadigd raken dat de doorbloeding van de spier gehinderd wordt. Ook is gekeken of spiervezeltypen die sneller beschadigen dan andere spiervezeltypen een minder goede capillaire voorziening. hebben. Tenslotte wilden wij het effect van hardlopen op de capillairen onderzoeken in de soleus spier in vivo (in het levende, genarcotiseerde dier). Om de wanden van capillairen zichtbaar te maken met behulp van een aangepaste lichtmicroscoop, de intravitaalmicroscoop, moest echter eerst een geschikte fluorescerende ("lichtende") stof gevonden worden.

Omdat we ontdekten dat de soleus spier zwol na het hardlopen, en omdat die zwelling een verhoging van de druk in de spier zou kunnen veroorzaken waardoor de doorbloeding belemmerd kan worden, is in een van de experimenten de druk in de spier tijdens de zwelling gemeten. Hiertoe zijn glaspipetten (holle glasnaaldjes) gemaakt waarvan de tip slechts enkele duizendsten van een millimeter dik was. De pipet werd voorzichtig in de spier geprikt waarna de druk werd gemeten.

Tijdens het onderzoek rees het vermoeden dat spierfilamenten van snelle en langzame spiervezels op verschillende afstanden van elkaar staan. De juistheid hiervan is onderzocht met behulp van een speciale toepassing van de electronenmicro. scoop, de electronendiffractie. 


\section{DE RESULTATEN}

De doorbloedingsmogelijkheden van de spier na hardlopen.

Eén dag na het hardlopen vonden we in de soleus spier van de rat inderdaad kleine ontstekingsreacties bij een deel van de spiervezels (zie figuur 12.4). De spiervezels die in de buurt van de ontstoken vezels lagen zagen er echter altijd volkomen normaal uit. Als de doorbloeding van de capillairen rond de ontstoken vezels inderdaad slecht zou zijn geweest, dan zouden we ook af en toe ontstekingen van naburige spiervezels verwachten. Verstopte, gezwollen of beschadigde capillairen werclen niet gevonden. De capillairen die tegen de ontstoken spiervezels aan lagen waren zelfs wijder geworden waardoor ze relatief juist veel bloed konden bevatten hetgeen het herstelproces van de beschadigde vezel zou kunnen bevorderen (hoofdstuk 3). De spiervezeltypen die gemakkelijker beschadigen door hardlopen dan andere spiervezeltypen bleken voorzien te zijn van een normale hoeveelheid capillairen van normale afmetingen (hoofdstuk 4 ).

Na het hardlopen bleek de soleus spier gezwollen te zijn. De zwelling, die 6 uur na de inspanning maximaal was, veroorzaakte geen verhoging van de druk in de spier. Dit betekent dat de doorbloeding van de spier niet gehinderd wordt door drukverhoging tijdens de zwelling (hoofdstuk 5).

Al deze gegevens wijzen er op dat er door het hardlopen weinig verandert aan de doorbloedingsmogelijkheden van de spier.

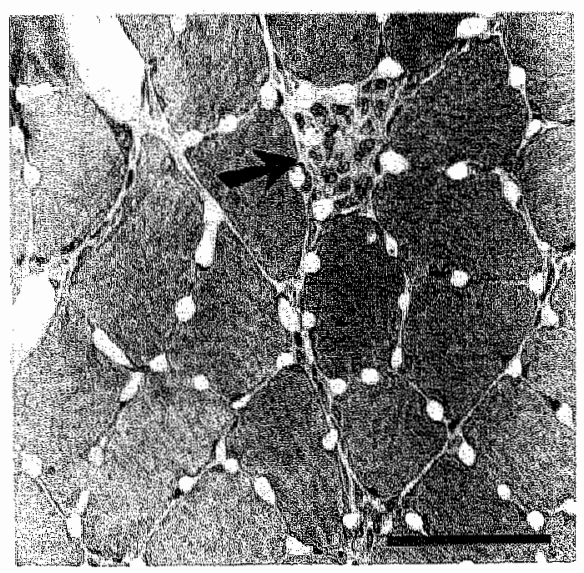

Fig. 12.4. Dwarsdoorsnede door de soleus spier 24 uur na hardlopen. Te zien zijn o. a. de spiervezels en de tussenliggende capillairen. De pijl wijst naar een ontstoken spiervezel. De beschadigde spiervezel zit vol met cellen die bij de ontstekingsreactie betrokken zijn. De naburige spiervezels zijn niet zichtbaar ontstoken. Streepje = $1 / 20 \mathrm{~mm}$. 
Bestuderen wan capillairen van de skeletspier in who.

Van de vele tientalle fluorescerende verbindingen die door ons zijn getest bleek er slechts één (pyronine B) geschikt te zijn om capillaire wanden zichtbaar te maken in de skeletspier (zie evt. figuur 7.2, blz. 87). Hierdoor kumen capillairen in de skeletspier in vivo nu beter zichtbaar gemaakt en onderzocht worden. Een nadeel van de gebruikte stof is echter dat bij injectie de bloeddruk sterk daalt hetgeen de toepassing van ervan beperkt. Verschillende stoffen die getest werden veroorzaakten een langdurige fluorescentie van het bloed of van celkernen waardoor ook deze beter herkend en onderzocht kunnen worden. Enkele geteste stoffen veroorzaakten bij aanstraling met licht een plaatselijke verstopping van de bloedbaan waardoor een klein weefselgebiedje van bloed afgesloten kan raken (hoofdstuk 7).

Met behulp van computeranalyse van videobeelden van fluorescerend bloed in de capillairen bleek het mogelijk te zijn de binnenste diameter van capillairen in spieren in vivo automatisch en met grotere nauwkeurigheid te bepalen (hoofdstuk 8; zie evt. figuur 8.2, blz. 95). De capillairen in de skelletspier van het genarcotiseerde dier bleken groter te zijn dan de capillairen die in de coupes van de spier werden waargenomen. De precieze oorzaak van dit verschil wordt momenteel onderzocht.

\section{Afstand tussen spierfilamenten.}

De spierfilamenten in snelle en sterke (type II) spiervezels bleken in alle door ons onderzochte spieren van de rat verder uiteen te liggen dan in langzame en zwakke (type I) vezels. Alhoewel men zou verwachten dat de sterkste en snelste spiervezels (type II) de meeste spierfilamenten per vierkante millimeter zouden hebben, is dit blijkbaar niet het geval. Waarschijnlijk zijn spierfilamenten van sterke en snelle spiervezels sterker en sneller dan spierfilamenten wan zwakkere, langzame spiervezels. De gevonden verschillen in de afstand tussen de spierfilamenten kunnen nu gebruikt worden om te bepalen of een spiervezel van het snelle of langzame type is waardoor ingewikkelde chemische technieken achterwege kunnen bljjven (hoofdstuk 6).

\section{TOT SLOT}

Mogelijk vraagt u zich af of de bovengenoemde experimenten nu wel zo nuttig zijn geweest. Het antwoord dat ik daarop geef is natuurlijk "ja". In de eerste plaats heeft het bestuderen van de problemen ons meer inzicht werschaft in de oorzaak van spiervezelontsteking, en mogelijk spierpijn, na overbelasting. Verder kan het onderzoek aan capillairen in spieren in wiwo nu met betere methodieken plaats vinden. Er zijn, tot nu toe onbekende, anatomische verschillen gevonden tussen langzame en snelle spiervezels die verschillen tussen de eigenschappen van 
spierfilamenten suggereren. Bovendien kan het onderzoek ook op andere gebieden vruchten afwerpen. Zo kan het bestuderen van de spierdoorbloeding ook van belang zijn voor bijwoorbeeld het hartonderzoek. Daarbij valt onder anderen te denken aan onderzoek naar hartinfarcten. Ook toevallige ontdekkingen kunnen van belang zijn. In een van de studies (hoofdstuk 7) zijn stoffen gevonden die gebruikt kunnen worden om, m.b.v. licht, de bloedtoevoer naar bepaalde weefselgebiedjes (b.v. kankerweefsel) af te sluiten.

Mocht $u$ na het lezen over de gevolgen van hardlopen, zoals spiervezelontstekingen en zelfs mogelijk overlijden, besloten hebben niet meer te sporten, bedenk dan dat er jaarlijks aanzienlijk meer mensen overlijden aan de gevolgen van het niet sporten dan aan gevolgen van wél sporten. 


\section{CURRICULUM VITAE}

Frank Peeze Binkhorst

1958: $\quad$ Geboren te Weert

1970-1977: V.W.O., Rijks Scholengemeenschap te Roermond

1977-1985: Studie Biologie aan Landbouw Unjversiteit, Wageningen.

1985-1986: Wetenschappelijk medewerker, vakgroep Experimentele Diermorfologie en Celbiologie, Landbouw Universiteit Wageningen.

1986-1989: Wetenschappelijk assistent, vakgroep Fysiologie, Rijksuniversiteit Limburg. 


\section{PUBLICATIONS}

1. Rombout, J.H.W.M., F.M. Peeze Binkhorst, J.J. Taverne-Thiele. Coexistence of PP- and glucagonlike immunoreactivity in endocrine cells of gut and pancreas of mouse and a stomachless fish Barbus conchonius. Cell Biology International Reports 9(6):520. 1985.

2. Rombout, J.H.W.M., C.P.M. van der Grinten, F.M. Peeze Binkhorst, J.J. Taverne-Thiele, and H. Sichooneveld. Immunocytochemical identification and localization of peptide hormones in the gastro-entero-pancreatic (GEP) endocrine system of the mouse and a stomachless fish, Barbus conchonius. Histochemistry 84:471-483. 1986.

3. Abad, M.E., F.M. Peeze Binkhorst, M.T. Elbal and J.H.W.M. Rombont. A comparative immunocytochemical study of the gastero-entero-pancreatic (GEP) endocrine system in a stormachless and a stomach-containing teleost. General and Comparative Endocrinology 66:123-136. 1987.

4. Rombout, J.H.W.M., M.E. Abad, F.M. Peeze Binkhorst, and J.J. Taverne-Thiele. Coexistence of pancreatic polypeptide (PP.) and glucagon-immunoreactivity in pancreatic endocrine cells of mouse. Histochemistry 87:1-6. 1987.

5. Peeze Binkhorst, F.M., H. Kuipers, P.M. Frederik, and G.-J. Tangelder. Capillary ultrastructure as related to fiber type in rat soleus muscle. Gesellschaft für Microcirculation e.v.. 12. Jahrestagung. Bern. 177-180. 1988.

6. Peeze Binkhorst, F.M., H. Kuipers, J. Heymans, P.M. Frederik, D.W. Slaaf, and R.S. Reneman. Exercise induced capillary changes in skeletal muscle. Ultramicroscopy 24:445. 1988.

7. Peeze Binkhorst, F.M., H. Kuipers, D.W. Slaaf, G.-I. Tangelder, P.M. Frederik, and R.S. Reneman. Is a disturbed microcirculation the cause of exercise induced muscle fiber degeneration? $\mathrm{A}$ morphometric study. Int. J. for Microcirc..Clin. Exp. 7:294.1988.

8. Peeze Binkhorst, F.M., H. Kuipers, G.-J. Tangelder, D.W. Staaf, P.M. Frederik, and R.S. Reneman. Exercise-induced muscle fiber degeneration: A microcirculatory cause? Pflugers Archiv. 412:1-2.S4. 1988.

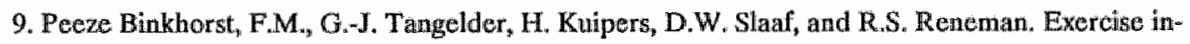
duced muscle fiber degeneration causes dilation of adjacent capillaries. Int. J. for Microcirc.: Clin. Exp. 155:S110. 1988.

10. Peeze Binkhorst, F.M., H. Kuipers, P.M. Frederik, D.W. Slaaf, and R.S. Reneman. Capillary ultrastructure as related to fiber type in rat soleus muscle. Int. J. Microcirc.: Clin. Exp. 8:107. 1988.

11. Peeze Binkhorst, F.M., H. Kuipers, J. Heymans, P.M. Frederik, D.W. Slaaf, G. -J. Tangelder, and R.S. Reneman. Exercise induced focal skeletal muscle fiber degeneration and capillary morphology. J. Appl. Physiol. 66(6):2857-2865. 1989.

12. Peeze Binkhorst, F.M., and P.M. Frederik. Myosin spacing in type I and type II fibers of rat soleus. muscle. Ultramicroscopy. 27:213. 1989. 
13. Kuipert, H., F.M. Peexe Binkhorst, G.-J. Tangelder, D.W. Slaaf, and P.M. Frederik. Dilation of capillaries adjacent to muscle fibers degenerated due to exercise. Medicine and Science in Sports and Exercise. 21(2):S81. 1989.

14. Peeze Binkhorst, F.M, H. Kuipers, G.-J. Tamgelder, D.W. Slaaf, and P.M. Frederik. Failure to confirm a microcirculatory cause for exercise induced muscle fiber degeneration. Medicine and Science in Sports and Exercise. 21(2):\$81. 1989.

1S. Peeze Binkhorst, F.M., H. Kuipers, G.-J. Tangelder, P.M. Frederik, D.W. Slaaf, and R.S. Reneman. Capillary (ultra)structure in relation to position and fiber type in rat soleus muscle. Submitted. 1989.

16. Peeze Binkhorst, F.M., D.W. Slaaf, G.-J. Tangelder, H. Kuipers, and R.S. Reneman. Exercise induced swelling of rat soleus muscle. Its relation to tissue pressure. Submitted. 1989.

17. Peeze Binkhorst, F.M., P.M. Frederik, M. Stuart, H. Kuipers, G.-J. Tangelder, and R.S. Reneman. Fiber type rolated thick filament spacing in plastic embedded muscle. Submitted. 1989.

18. Kuipers, H., F.M. Peeze Binkhorst, F. Hartgens, and J.A.G. Wijnen. The effect of anabolic steroids on skeletal muscle ultrastructure in bodybuilders. Submitted. 1989.

19. Peeze Binkhorst, F.M., G.-J. Tangelder, D.W. Slaaf, H. Kuipers, and R.S. Reneman. Fluorescent labeling of capillary endothelium in wiwo. In prep. 1989.

20. Peeze Binkhorst, F.M., G.-J. Tangelder, D.W. Slaaf, H. Kuipers, and R.S. Reneman. Measurement of skeletal muscle capillary diameter in vivo. In prep. 1989. 
In dit nawoord wil ik iedereen bedanken die op een of andere manier heeft bijgedragen aan de totstandkoming van dit proefschrift.

In de eerste plaats de medewerkers van het laboratorium voor Microcirculatie dat in de afgelopen periode mijn uitvalsbasis is geweest. Jullie hebben op een altijd hulpvaardige en vaak vriendschappelijke manier mijn verblijf daar tot een bijzonder leerzame ervaring gemaakt. Ook de andere medewerkers $(\mathrm{m} / \mathrm{v})$ van de vakgroep Fysiologie, en niet in de laatste plaats de secretaresses, wil ik voor hun bijdrage bedanken.

De medewerkers van het laboratorium voor Electronenmicroscopie. Behalve een onbaatzuchtige technische ondersteuning die ik van jullie heb gekregen heb ik bij jullie ook een bijzonder prettige tijd gehad. Het spijt me dat mijn gejaagdheid jullie concentratie op het schaakgebeuren soms verstoord heeft.

Verder wil ik de medewerkers van de laboratoria voor Anatomie, Pathologie en Farmacologie bedanken voor alle steun die jullie, vaak met een ongewone vanzelfsprekendheid, hebben gegeven.

Vroedvrouwen in opleiding. Jullie les te mogen geven was een van mijn leukste en leerzaamste ervaringen van de afgelopen jaren. De vreemde glimlach die ik bij mijn collega's ontdekte als ik over jullie sprak heb ik nooit helemaal begrepen.

In het bijzonder wil ik Harm, Geert-Jan en Peter niet in de laatste plaats bedanken voor hun persoonlijke steun gedurende de afgelopen jaren. Jullie begrijpen dat voor het bereiken van je doel een goede sfeer minstens zo belangrijk is als een goede inzet. Mijn paranimfen Mirjam en Huub, die bij het schrijven van dit stukje nog niet weten dat ze paranimf gaan worden, zijn niet alleen mijn lotgenoten geweest maar ook persoonlijke vrienden geworden.

Tenslotte rest mij alle druk bezette leden van de beoordelingscomissie hartelijk te danken voor het kritissch doorlezen van het manuscript.

Vele namen liggen mij nog voor op de lippen. Ik hoop dat jullie weten dat ik jullie, alhoewel niet met name genoend, niet vergeten ben.

Een prettige tijd verder.

Frank. 